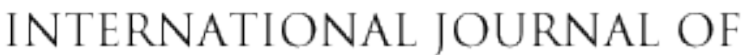 GYNECOLOGICAL CANCER
}

\section{ESGO/ESTRO/ESP Guidelines for the management of patients with endometrial carcinoma}

\begin{tabular}{|c|c|}
\hline Journal: & International Journal of Gynecological Cancer \\
\hline Manuscript ID & ijgc-2020-002230 \\
\hline Article Type: & Original research \\
\hline $\begin{array}{r}\text { Date Submitted by the } \\
\text { Author: }\end{array}$ & 07-Nov-2020 \\
\hline Complete List of Authors: & $\begin{array}{l}\text { Concin, Nicole; Department of Gynecology and Obstetrics; Innsbruck } \\
\text { Medical Univeristy } \\
\text { Matias-Guiu, Xavier; Hospital Universitari Arnau de Vilanova, Department } \\
\text { of Pathology } \\
\text { Vergote, Ignace; Leuven Cancer Institute, Catholic University Leuven, } \\
\text { Department of Gynecology and Obstetrics, Gynecologic Oncology } \\
\text { Cibula, David; University of Prague, Dept of Obstetrics and Gynecology } \\
\text { Mirza, Mansoor; Rigshospitalet; Copenhagen University Hospital, } \\
\text { Department of Oncology } \\
\text { Marnitz, Simone; Medical Faculty of the University of Cologne, } \\
\text { Department of Radiooncology } \\
\text { Ledermann, Jonathan; UCL Cancer Institute, University College } \\
\text { Bosse, Tjalling; Leids Universitair Medisch Centrum } \\
\text { Chargari, Cyrus; Institut Gustave-Roussy, Department of radiation } \\
\text { oncology } \\
\text { Fagotti, Anna; Fondazione Policlinico Universitario A. Gemelli IRCCS, } \\
\text { Division of Gynecologic Oncology } \\
\text { Fotopoulou, Christina; Imperial College London Faculty of Medicine, } \\
\text { Gynaecologic Oncology } \\
\text { Gonzalez Martin, Antonio; Clinica Universidad de Navarra, Medical } \\
\text { Oncology; } \\
\text { LAX, Sigurd; Hospital Graz Sud-West, Pathology } \\
\text { Lorusso, Domenica; Policlinico Gemelli } \\
\text { Marth, Christian; Innsbruck Medical University, Obstetrics and } \\
\text { Gynecology } \\
\text { Morice, Philippe; Gustave Roussy, Surgery } \\
\text { Nout, Remi; Leiden University Medical Center, Radiation Oncology } \\
\text { O'Donnell, Dearbhaile; St James Hospital, Department of Medical } \\
\text { Oncology } \\
\text { Querleu, Denis; Institut Bergonie, Surgery } \\
\text { Raspollini, Maria Rosaria; Azienda Ospedaliero Universitaria Careggi } \\
\text { Sehouli, Jalid; Charité - Universitätsmedizin Berlin, corporate member of } \\
\text { Freie Universität Berlin, Humboldt-Universität zu Berlin and Berlin } \\
\text { Institute of Health, Department of Gynecology with Center for } \\
\text { Oncological Surgery, Campus Virchow Klinikum } \\
\text { Sturdza, Alina; Medical University of Vienna, Department of Radiation } \\
\text { Oncology, Comprehensive Cancer Center, Christian Doppler Laboratory } \\
\text { for Medical Radiation Research for Radiation Oncology } \\
\text { Taylor, Alexandra; Royal Marsden Hospital, Gynaecology }\end{array}$ \\
\hline
\end{tabular}




\begin{tabular}{|c|l|}
\hline & Westermann, Anneke; Amsterdam University Medical Centres, \\
& Department of Medical Oncology \\
& Wimberger, Pauline; Technische Universitat Dresden Medizinische \\
& Fakultat Carl Gustav Carus, Gyncology and Obstetrics \\
& Colombo, Nicoletta; European Institute of Oncology, Medical Gynecologic \\
& Oncology Unit; University of Milan Bicocca; Milan; Italy \\
& Planchamp, François; Institut Bergonie, Clinical Research Unit \\
& Creutzberg, Carien; LUMC, Clinical Oncology \\
\hline Keywords: & Uterine Cancer, Surgery, Radiation, Pathology \\
\hline \multicolumn{2}{|l}{} \\
\hline
\end{tabular}

\section{SCHOLARONE \\ Manuscripts}




\title{
ESGO/ESTRO/ESP Guidelines for the management of patients with endometrial carcinoma*
}

\begin{abstract}
Authors: Nicole Concin ${ }^{1}$, Xavier Matias-Guiu ${ }^{2}$, Ignace Vergote ${ }^{3}$, David Cibula ${ }^{4}$, Mansoor Raza Mirza ${ }^{5}$, Simone Marnitz ${ }^{6}$, Jonathan A. Ledermann ${ }^{7}$, Tjalling Bosse ${ }^{8}$, Cyrus Chargari ${ }^{9}$, Anna Fagotti ${ }^{10}$, Christina Fotopoulou ${ }^{11}$, Antonio González-Martín ${ }^{12}$, Sigurd F. Lax ${ }^{13}$, Domenica Lorusso ${ }^{14}$, Christian Marth ${ }^{15}$, Philippe Morice $^{16}$, Remi A. Nout ${ }^{17}$, Dearbhaile E. O'Donnell ${ }^{18}$, Denis Querleu ${ }^{19}$, Maria Rosaria Raspollini ${ }^{20}$, Jalid Sehouli $^{21}$, Alina E. Sturdza ${ }^{22}$, Alexandra Taylor ${ }^{23}$, Anneke M. Westermann ${ }^{24}$, Pauline Wimberger ${ }^{25}$, Nicoletta Colombo ${ }^{26}$, François Planchamp ${ }^{27}$, Carien L. Creutzberg ${ }^{28}$
\end{abstract}

A European consensus conference on endometrial carcinoma was held in 2014 to produce multidisciplinary evidence-based guidelines on selected questions. Given the large body of literature on the management of endometrial carcinoma published since 2014, the European Society of Gynaecological Oncology (ESGO), the European SocieTy for Radiotherapy \& Oncology (ESTRO) and the European Society of Pathology (ESP) jointly decided to update these evidence-based guidelines and to cover new topics in order to improve the quality of care for women with endometrial carcinoma across Europe and worldwide.

ESGO/ESTRO/ESP nominated an international multidisciplinary development group consisting of practicing clinicians and researchers who have demonstrated leadership and expertise in the care and research of endometrial carcinoma (27 experts across Europe). To ensure that the guidelines are evidence-based, the literature published since 2014, identified from a systematic search was reviewed and critically appraised. In the absence of any clear scientific evidence, judgment was based on the professional experience and consensus of the development group. The guidelines are thus based on the best available evidence and expert agreement. Prior to publication, the guidelines were reviewed by 191 independent international practitioners in cancer care delivery and patient representatives.

The guidelines comprehensively cover endometrial carcinoma staging, definition of prognostic risk groups integrating molecular markers, pre- and intra-operative work-up, fertility preservation, management for early, advanced, metastatic, and recurrent disease and palliative treatment. Principles of radiotherapy and pathological evaluation are also defined.

Key Words: Endometrial carcinoma, Guidelines, Staging, Molecular markers, Management

Affiliations: ${ }^{1}$ Department of Obstetrics and Gynecology, Medical University of Innsbruck, Innsbruck (Austria); Department of Gynecology and Gynecological Oncology, Evangelische Kliniken Essen-Mitte, Essen (Germany); ${ }^{2}$ Department of Pathology, Hospital UniversitariArnau de Vilanova and Hospital Universitari de Bellvitge, Irblleida, Idibell, Universities of Lleida and Barcelona, Ciberonc (Spain); ${ }^{3}$ Department of Gynecologic Oncology, Leuven University Hospitals, Leuven Cancer Institute, Leuven (Belgium, European Union); ${ }^{4}$ Department of Obstetrics and Gynecology, General University Hospital, Prague, Charles University, Prague (Czech Republic); ${ }^{5}$ Department of Oncology, Rigshospitalet, Copenhagen University Hospital, Copenhagen (Denmark); ${ }^{6}$ Department of Radiation Oncology, University of Cologne, Cologne (Germany); ${ }^{7}$ Department of Oncology and Cancer Trials, UCL Cancer Institute, London (United Kingdom); ${ }^{8}$ Department of Pathology, Leiden University Medical Center, Leiden (The Netherlands); ${ }^{9}$ Department of Radiation Oncology, Institut Gustave Roussy, Villejuif, France; ${ }^{10}$ Division of Gynecologic Oncology, Dipartimento Scienzedella Salute della Donna e del Bambino, Fondazione Policlinico Universitario A. Gemelli IRCCS, Roma (Italy); ${ }^{11}$ Department of Gynecologic Oncology, Imperial College London, London (United Kingdom); ${ }^{12}$ GEICO and Department of Medical Oncology, Clinica Universidad de Navarra, Madrid (Spain); ${ }^{13}$ Department of Pathology, Hospital Graz II, Graz and Johannes Kepler University Linz, School of Medicine (Austria); ${ }^{14}$ Scientific Directorate and Division of Gynecologic Oncology, Dipartimento Scienzedella Salute della Donna e del Bambino, Fondazione Policlinico Universitario A. Gemelli IRCCS, Roma (Italy); ${ }^{15}$ Department of Obstetrics and Gynecology, Innsbruck Medical University, Innsbruck (Austria); ${ }^{16}$ Department of Gynaecological Surgery, Institut Gustave Roussy, Villejuif (France); ${ }^{17}$ Department of Radiotherapy, Leiden University Medical Center, Leiden (The Netherlands); ${ }^{18}$ Department of Medical Oncology, St. James Hospital, Dublin (Ireland); ${ }^{19}$ Department of Surgery, Institut Bergonie, Bordeaux (France); ${ }^{20}$ Department of Histopathology and Molecular Diagnostics, University Hospital, Careggi, Florence (Italy); ${ }^{21}$ Department of Gynecology with center for oncological surgery, Charite-
Universitätsmedizin Berlin, Berlin (Germany); ${ }^{22}$ Department of Radiation Oncology, Medical University of Vienna, Vienna (Austria); ${ }^{23}$ Department of GynaeOncology, The Royal Marsden Hospital , London (United Kingdom): ${ }^{24}$ Department of Medical Oncology, Amsterdam University Medical Center, Amsterdam (The Netherlands); ${ }^{25}$ Department of Gynecology and Obstetrics, Technische Universität Dresden, Dresden (Germany); ${ }^{26}$ Division of Medical Gynecologic Oncology, European Institute of Oncology, IRCCS and University of Milan-Bicocca, Milan (Italy); ${ }^{27}$ Clinical research Unit, Institut Bergonie, Bordeaux (France); ${ }^{28}$ Department of Radiation Oncology, Leiden University Medical Center, Leiden (The Netherlands).

Address correspondence and reprint requests to Nicole Concin, MD, PhD Department of Obstetrics and Gynecology, Medical Universtiy of Innsbruck, Innrain 52, 6020 Innsbruck, Austria.

Email: nicole.concin@i-med.ac.at

Initiated through ESGO the decision to develop multidisciplinary guidelines has been made jointly by the ESGO, ESTRO, and ESP. The ESGO has provided administrative support. The ESGO, ESTRO and ESP are nonprofit knowledgeable societies.

The development group (including all authors) is collectively responsible for the decision to submit for publication. Nicole Concin (chair), Carien L. Creutzberg (cochair), Xavier Matias-Guiu (co-chair) and François Planchamp (methodologist) have written the first draft of the manuscript. All other contributors have actively given personal input, reviewed the manuscript, and have given final approval before submission.

* These guidelines statements were developed by ESGO, ESTRO and ESP and are published in the International Journal of Gynecological Cancer, Radiotherapy \& Oncology and the Virchows Archiv. 


\section{INTRODUCTION}

Endometrial carcinoma is the most common gynaecological cancer in Europe, with a 5-year prevalence of $34.7 \%(445,805 \text { cases })^{1}$. The estimated number of new endometrial carcinoma cases in Europe in 2018 was 121,578 with 29,638 deaths, and incidence has been rising with aging and increased obesity of the population. The EUROCARE-5 study, published in 2015, reported a 5-year relative survival of $76 \%$ for European women diagnosed with endometrial carcinoma in 2000-2007, ranging from $72.9 \%$ in Eastern Europe to $83.2 \%$ in Northern Europe ${ }^{2}$. The observed geographic difference might be partially attributable to tangible differences in endometrioid subtype prevalence among regions. Furthermore, differences in patient characteristics and histopathological features of the disease impact both on patient prognosis and the recommended treatment approach.

A consensus conference including representation from the European Society of Medical Oncology (ESMO), the European Society of Gynaecological Oncology (ESGO) and the European SocieTy for Radiotherapy \& Oncology (ESTRO) was held in 2014 with the aim to produce multidisciplinary evidence-based guidelines on 12 selected questions in order to complement the ESMO clinical practice guidelines previously published $^{3-6}$. The ESGO, the ESTRO and the European Society of Pathology (ESP) jointly decided to update these evidence-based guidelines and moreover to cover new topics in order to provide comprehensive guidelines on all relevant issues of diagnosis and treatment in endometrial carcinoma in a multidisciplinary setting. These guidelines are intended for use by gynaecological oncologists, general gynaecologists, surgeons, radiation oncologists, pathologists, medical and clinical oncologists, radiologists, general practitioners, palliative care teams, and allied health professionals.

\section{RESPONSIBILITIES}

These guidelines are a statement of evidence and consensus of the authors regarding their views of currently accepted approaches for the management of patients with endometrial carcinoma. Any clinician applying or consulting these guidelines is expected to use independent medical judgment in the context of individual clinical circumstances to determine any patient's care or treatment. These guidelines make no warranties of any kind regarding their content, use, or application and the authors disclaim any responsibility for their application or use in any way. 


\section{METHODS}

The guidelines were developed using a five-step processas defined by the ESGO Guideline Committee (see Figure 1). The strengths of the process include creation of a multidisciplinary international development group, use of scientific evidence and international expert consensus to support the guidelines, and use of an international external review process (physicians and patients). This development process involved three meetings of the international development group, chaired by Professor Nicole Concin (Medical University of Innsbruck, Innsbruck, Austria/Evangelische Kliniken Essen-Mitte, Essen, Germany, for ESGO), Professor Carien L. Creutzberg (Leiden University Medical Center, Leiden, The Netherlands, for ESTRO), and Professor Xavier Matias-Guiu (Hospital UniversitariArnau de Vilanova, University of Lleida, Lleida/Hospital Universitari de Bellvitge, University of Barcelona, Barcelona, Spain, for ESP).

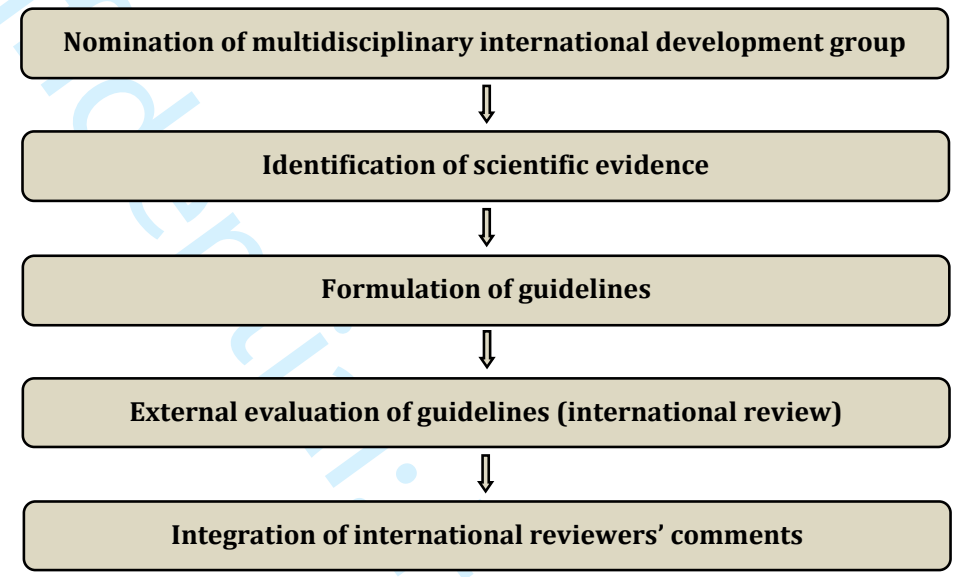

Figure 1. Development process

ESGO/ESTRO/ESP nominated practising clinicians who are involved in the management of endometrial carcinoma patients and have demonstrated leadership in clinical management of patients through research, administrative responsibilities, and/or committee membership to serve on the expert panel. The objective was to assemble a multidisciplinary panel and it was therefore essential to include professionals from relevant disciplines (gynaecological oncology and gynaecology, medical, clinicaland radiation oncology, pathology) to contribute to the validity and acceptability of the guidelines. To ensure that the statements were evidence based, the current literature was reviewed and critically appraised. A systematic literature review of relevant studies published between January 2014 and june 2019 was carried out using the MEDLINE database (see Appendix 1). The literature search was limited to publications in English. Priority was given to high-quality systematic reviews, meta-analyses, and randomised controlled trials, but studies of lower levels of evidence were also evaluated. The search strategy excluded editorials, letters, and in vitro studies. The reference list of each identified article was also reviewed for other potentially relevant articles.

The development group developed guidelines for all the topics. The guidelines were retained if they were supported by sufficiently high level scientific evidence and/or when a large consensus among experts was obtained. An adapted version of the "Infectious Diseases Society of America-United States Public Health Service Grading System" was used to define the level of evidence and grade of recommendation for each of the recommendations ${ }^{7}$ (see Figure 2). In the absence of any clear scientific evidence, judgment was based on the professional experience and consensus of the development group. 


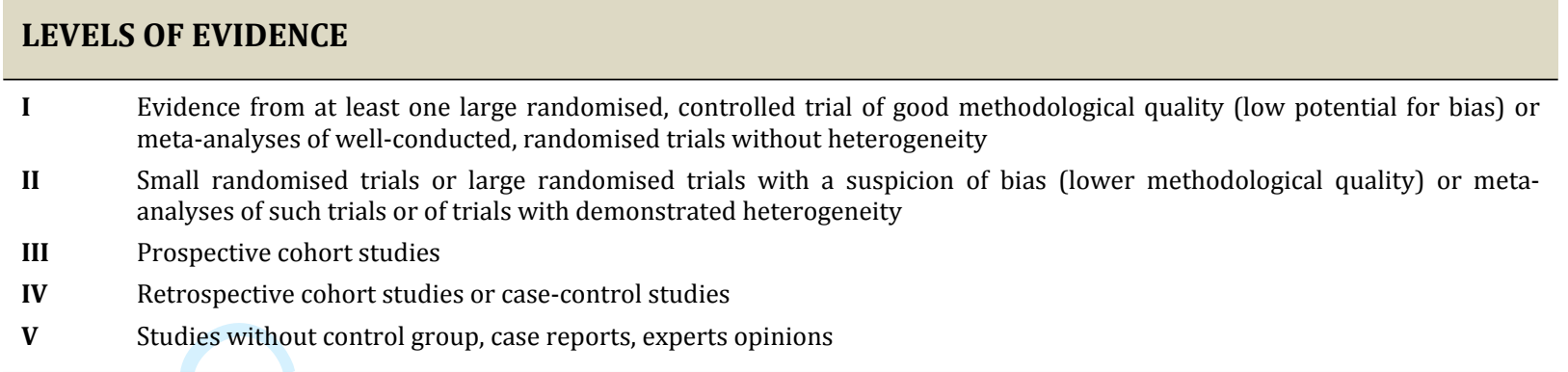

\section{GRADES OF RECOMMENDATIONS}

\begin{tabular}{ll}
\hline A & Strong evidence for efficacy with a substantial clinical benefit, strongly recommended \\
B & Strong or moderate evidence for efficacy but with a limited clinical benefit, generally recommended \\
C & Insufficient evidence for efficacy or benefit does not outweigh the risk or the disadvantages (adverse events, costs, ...), \\
& optional \\
D & Moderate evidence against efficacy or for adverse outcome, generally not recommended \\
E & Strong evidence against efficacy or for adverse outcome, never recommended \\
\hline
\end{tabular}

Figure 2. Levels of evidenceand grades of recommentions

ESGO/ESTRO/ESP established a large multidisciplinary panel of practicing clinicians that provide care to endometrial carcinoma patients to act as independent expert reviewers for the guidelines developed. These reviewers were selected according to their expertise, had to be still involved in clinical practice, and were from different European and non-European countries to ensure global perspective. Endometrial carcinoma patients were also included. These independent reviewers were asked to evaluate each recommendation according to its relevance and feasibility in clinical practice (only physicians), so that comprehensive quantitative and qualitative evaluations of the guidelines were completed. Patients were asked to evaluate qualitatively each recommendation (according to their experience, personal perceptions etc.). Evaluations of the external reviewers $(\mathrm{N}=191)$ were pooled and discussed by the international development group before finalising the guidelines. The list of the 191 external reviewers is available in Appendix 2.

\section{GENERAL RECOMMENDATIONS}

- Planning of staging and treatment should be made on a multidisciplinary basis (generally at a tumour board meeting, composed according to local guidelines) and based on the comprehensive and precise knowledge of prognostic and predictive factors for outcome, morbidity, and quality of life [V, A].

- Patients should be carefully counselled about the suggested diagnostic and treatment plan and potential alternatives, including risks and benefits of all options [V, A].

- Treatment should be undertaken in a specialised centre by a dedicated team of specialists in the diagnosis and management of gynaecological cancers, especially in high risk and/or advanced stage disease $[\mathrm{V}, \mathrm{A}]$.

\section{IDENTIFICATION AND SURVEILLANCE OF WOMEN WITH A PATHOGENIC GERMLINE VARIANT IN A LYNCH SYNDROME- ASSOCIATED GENE}

Approximately $3 \%$ of all endometrial carcinomas and about $10 \%$ of mismatch repair deficient (MMRd)/microsatellite unstable endometrial carcinomas are causally related to germline mutations of one of the MMR genes MLH1, PMS2, MSH2 and $M S H 6^{8}$. Testing for MMR status/microsatellite instability 
(MSI) in endometrial carcinoma patients has been shown to be relevant for four reasons: 1) diagnostic, as MMRd/MSI is considered a marker for endometrioid-type endometrial carcinoma, 2) pre-screening to identify patients at higher risk for having Lynch syndrome, 3) prognostic as identified by The Cancer Genome Atlas (TCGA, see below for molecular classification), and 4) predictive for potential utility of immune checkpoint inhibitor therapy. The International Society of Gynecologycal Pathology (ISGyP) has recommended testing for MMR status/MSI in all endometrial carcinoma samples, irrespective of age ${ }^{9}$. This has also been recommended in other society statements and recommendations, such as the Manchester International Consensus Group recommendations, whenever resources are available ${ }^{10}$.

The preferred approach (widely available and cost-effective) to identifying patients with a higher chance of having Lynch syndrome, is by MMR-immunohistochemistry (IHC) on well preserved tumour tissue. MMR-IHC is a reliable method to assess MMR status, and in addition provides information on the altered gene/protein. ISGyP guidelines therefore recommend MMR-IHC as the preferred test ${ }^{9}$. MMR-IHC consists of the assessment of the expression of four MMR proteins (MLH1, PMS2, MSH6, and MSH2). A simplified two-antibody (PMS2 and MSH6) approach has been proposed as a cost-effective alternative ${ }^{11-13}$. This procedure still requires performing MLH1 and MSH2 IHC in cases with any abnormal staining of PMS2 and/or MSH6. Molecular analyses for the microsatellite status (MSI-test) are an alternative, but are more laborious, require non-neoplastic tissue, are more expensive, and do not provide information on the gene affected. For optimal preselection of patients at risk for having Lynch syndrome, both approaches require the analysis of MLH1 promoter methylation status in cases with loss of MLH1/PMS2 expression. Testing for MMRd by IHC or MSI by PCR-based methods, does not allow direct identification of Lynch syndrome patients, since MMRd/MSI is frequently due to sporadic events such as bi-allelic somatic mutations or hypermethylation. In absence of hypermethylation, referral to genetic counseling is recommended to evaluate the presence of a germline mutation. When familial history is highly suspicious of Lynch syndrome, genetic counselling is recommended independent of the MMR status.

The cumulative incidences for cancer depend on the specific mutation in women with Lynch sydrome. For endometrial carcinoma, the cumulative incidences at 70 years are 34\%, 51\%, 49\% and 24\% for $M L H 1$, MSH2, MSH6 and PMS2 mutation carriers, respectively, and for ovarian cancer 11\%, 15\%, 0\% and $0 \%$, respectively ${ }^{14}$. Furthermore, the age ofcancer onset in Lynch syndrome varies among specific mutated genes and type of mutations ${ }^{15}$. As part of a retrospective study, Lachiewicz et al. reported a risk of any occult malignancy during prophylactic surgery for women with Lynch syndrome or Hereditary NonPolyposis Colorectal Cancer to be up to $17 \%{ }^{16}$. Thus, these patients should be counselled about the risk of detection of gynaecological cancer at prophylactic surgery.

\section{$\underline{\text { Recommendations: }}$}

- $\quad$ To identify patients with Lynch syndrome and triage for germline mutational analysis, MMR IHC (plus analysis of MLH1 promotor methylation status in case of immunohistochemical loss of MLH1/PMS2 expression) or MSI tests should be performed in all endometrial carcinomas, irrespective of histologic subtype of the tumour [III, B].

- Endometrial carcinoma patients identified as having an increased risk of Lynch syndrome, should be offered genetic counselling [III, B].

- Surveillance for endometrial carcinoma in Lynch syndrome mutation carriers should in general start at the age of 35 years, however individual factors need to be taken into consideration (tailored surveillance programmes). The decision on the starting age of surveillance should integrate knowledge on the specific mutation and history of onset of events in the family [IV, B].

- Surveillance of the endometrium by annual transvaginal ultrasound (TVUS) and annual or biennial biopsy until hysterectomy should be considered in all Lynch syndrome mutation carriers [IV, B]. 
- Hysterectomy and bilateral salpingo-oophorectomy, to prevent endometrial and ovarian cancer, should be performed at the completion of childbearing and preferably before the age of 40 years. All the pros and cons of prophylactic surgery must be discussed including the risk of occult gynaecological cancer detection at prophylactic surgery. Oestrogen replacement therapy should be suggested if bilateral salpingo-oophorectomy is performed in premenopausal women [IV, B].

\section{MOLECULAR MARKERS FOR ENDOMETRIAL CARCINOMA DIAGNOSIS AND AS DETERMINANTS FOR TREATMENT DECISIONS}

Different types of endometrial carcinoma have specific histological and molecular features, precursor lesions and natural histories. Conventional pathologic analysis remains an important tool for tumour stratification, but suffers from interobserver variation. Different groups have applied a diagnostic algorithm using three immunohistochemical markers (p53, MSH6 and PMS2), and one molecular test (mutation analysis of the exonuclease domain of POLE) to identify prognostic groups analogous to the TCGA molecular-based classification ${ }^{17-21}$. The feasibility of this approach was confirmed by a large number of publications that have all consistently reported prognostic relevance particularly in high-grade and high-risk tumours in several independent cohorts and prospective clinical trials ${ }^{22}$. To apply this molecular classification, all these diagnostic tests need to be performed. Performing one of the surrogate marker tests in isolation is insufficient, as a combination of positive tests can occur in approximatively $5 \%$ of all carcinomas. The diagnostic algorithm to classify these so-called "multiple classifiers" has been described recently ${ }^{23,24}$. In addition, endometrial carcinoma should only be classified as POLE-mutated (POLEmut), when pathogenic variants of POLE are identified in the gene's exonuclease domain ${ }^{25,26}$.

This surrogate marker approach to the molecular-based classification has been demonstrated to be prognostically informative in low, intermediate-, and high-risk endometrial carcinoma. Smaller studies showed that the molecular classification isalso be applicable to non-endometrioid tumours, including serous, clear cell, undifferentiated carcinomas and uterine carcinosarcomas. For adjuvant treatment recommendations, the molecular classification seems to be particularly relevant in the context of highgrade and/or high-risk endometrial carcinomas. Application of the molecular classification in high-grade and/or high-risk endometrial carcinomas shows that there is a group of patients with an excellent prognosis, i.e. the POLEmut tumours, and a group with poor prognosis, i.e. the p53-abnormal (p53abn) tumours. Endometrial carcinomas with MMRd or nonspecific molecular profile (NSMP) have an intermediate prognosis. However, the molecular surrogate is not perfect. Immunohistochemical demonstration of p53abn is a good but not perfect surrogate of TP53 mutation. Furthermore, a small proportion of high copy number tumours do not show TP53 mutations. To minimize these limitations, an integrated analysis combining traditional pathologic and molecular results seems ideal. In low risk endometrioid carcinomas, the molecular classification may not be required ${ }^{27,28}$.

The proposed molecular classification of endometrial carcinoma is clinically feasible using a limited set of diagnostic tests. Using this novel classification is encouraged. All diagnostic tests should be performed in conjunction due to the occurrence of "double classifiers" ${ }^{23}$. Clinical management may be particularly impacted by the molecular classification in scenarios where adjuvant chemotherapy is considered (high grade/high-risk disease). Thus, these cases should be prioritized when there is a lack of sufficient resources to perform this classification on all endometrial carcinomas. If molecular classification tools are not available, endometrial carcinoma classification should be based on traditional pathological features. There is still room for other biomarkers that may be potentially useful in the big group of low-grade endometrioid carcinoma with NSMP, such as L1CAM expression or mutations in CTNNB1 $^{29-32}$. 


\section{Recommendations:}

- Molecular classification is encouraged in all endometrial carcinomas, especially high-grade tumours $[\mathrm{IV}, \mathrm{B}]$.

- $\quad$ POLE mutation analysis may be omitted in low-risk and intermediate risk endometrial carcinoma with low grade histology [IV, C].

\section{DEFINITION OF PROGNOSTIC RISK GROUPS INTEGRATING MOLECULAR MARKERS}

There is overwhelming evidence that traditional pathologic features, such as histopathologic type, grade, myometrial invasion, and lymphovascular space invasion (LVSI), are important in assessing prognosis, as recommended in the ISGyP guidelines ${ }^{9}$. Histopathological typing should be performed according to the World Health Organization (WHO) Classification of tumours ( $5^{\text {th }}$ edition $)^{33}$. A binary International Federation of Gynecology and Obstetrics (FIGO) grading is recommended, which considers grade 1 and grade 2 carcinomas as low-grade and grade 3 carcinomas as high-grade. For the assessment of myometrial invasion, account needs to be taken of the endo-myometrial junction which is undulating ${ }^{34}$. Focal LVSI is defined by the presence of a single focus around the tumour, substantial LVSI as multifocal or diffuse arrangement of LVSI or the presence of tumour cells in 5 or more lymphovascular spaces. The molecular classification adds another layer of information to the conventional morphologic features and therefore should be integrated in the pathologic report.

\section{Recommendations:}

- Histopathological type, grade, myometrial invasion and LVSI (no/focal/substantial) should be recorded in all patients with endometrial carcinoma $[\mathrm{V}, \mathrm{A}]$.

- The definition of prognostic risk groups is presented in the Figure 3 for both situations, when Molecular Classification is known or unknown. 
node metastases during preoperative staging probably reflects the need for a sufficient number of neoplastic cells to induce ${ }^{18} \mathrm{~F}$-fluoro-2-deoxy-D-glucose hypermetabolism ${ }^{89-100}$. The usefulness of maximal standardized uptake value in classifying patients into pre-defined riskgroups is limited ${ }^{101}$. A preoperative computed tomography (CT) scan has a clinical utility in patients with endometrial carcinoma in detecting metastatic disease $\mathrm{e}^{102,103}$.

Frozen section of endometrial biopsy material is obsolete. Myometrial invasion should not be assessed by frozen section because of poor reproducibility and agreement with definitive paraffin sections. Since sentinel node biopsy is increasingly used, the need for intraoperative assessment of myometrial invasion has become less important. Moreover, some of the biomarkers that have been proposed require an optimal management of the surgical specimen with high quality of pre-analytical issues, such as appropriate fixation conditions. Performing frozen sections can lead to incorrect control of pre-analytical conditions, sometimes even leading to incorrect assessment of LVSI, due to artifactual displacement of tumour cells into vascular spaces during processing. In addition, the freezing of tissue before fixation and further processing interferes with an optimal pre-analytical procedure required for standardized histopathological diagnosis.

\section{Recommendations:}

- Histopathological tumour type and grade in endometrial biopsy is required [IV, A].

- Pre-operative mandatory work-up includes: family history; general assessment and inventory of comorbidities, geriatric assessment, if appropriate; clinical examination, including pelvic examination; expert transvaginal or transrectal ultrasound or pelvic MRI [IV, C].

- Depending on clinical and pathological risk, additional imaging modalities (thoracic, abdominal and pelvic CT scan, MRI, PET scan or ultrasound) should be considered to assess ovarian, nodal, peritoneal and other sites of metastatic disease [IV, C].

- Intra-operative frozen section is not encouraged for myometrial invasion assessment because of poor reproducibility and interference with adequate pathological processing [IV, A].

\section{EARLY STAGE DISEASE}

\section{Surgical management of apparent stage I/II endometrial carcinomas Minimally invasive approach}

Two randomised prospective studies comparing minimally invasive with open surgeries showed similar survival with quicker recovery with the minimally invasive approach ${ }^{104,105}$. More recently, pooled analyses of randomised prospective studies including notably these 2 studies, and multiple retrospective and prospective studies support also the use of minimally invasive surgery for patients including those with high risk endometrial carcinoma ${ }^{106-171}$.

\section{Recommendations:}

- Minimally invasive surgery is the prefered surgical approach, including patients with high risk endometrial carcinoma [I, A].

- Any intraperitoneal tumour spillage, including tumour rupture or morcellation (including in a bag), should be avoided [III, B].

- If vaginal extraction risks uterine rupture, other measures should be taken (e.g. mini laparotomy, use of endobag) [III, B]. 
- Tumours with metastases outside the uterus and cervix (excluding lymph node metastases) are relative contra-indications for minimally invasive surgery [III, B].

\section{$\underline{\text { Standard surgical procedures }}$}

In a randomised controlled trial comparing modified radical (Piver-Rutledge class II) hysterectomy to the standard extrafascial (Piver-Rutledge class I) or simple total hysterectomy in stage I endometrial carcinoma, Signorelli et al. showed no differences in locoregional control and survival ${ }^{172}$. The high risk of microscopic omental metastases in stage I serous and undifferentiated endometrial carcinoma and in carcinosarcoma suggests that omentectomy should be part of staging surgery in these patients ${ }^{173}$. The low rate of omental metastases in apparent clinical stage I endometrioid and clear cell carcinoma does not justify the procedure ${ }^{174}$. Although the risk of having occult (microscopic) omental metastases in carcinosarcoma is around 6\%, staging omentectomy in these women is suggested. Identification of these cases will allow inclusion of patients with advanced stage disease into clinical trials ${ }^{175}$. Positive peritoneal cytology correlates with poor prognostic factors and poor survival; however it is not part of FIGO staging and unclear if this should influence treatment decisions ${ }^{176-178}$.

\section{Recommendations:}

- Standard surgery is total hysterectomy with bilateral salpingo-oophorectomy without vaginal cuff resection [II, A].

- Staging infracolic omentectomy should be performed in clinical stage I serous endometrial carcinoma, carcinosarcoma and undifferentiated carcinoma. It can be omitted in clear cell and endometrioid carcinoma in stage I disease [IV, B].

- Surgical restaging can be considered in previously incompletely staged patients with high intermediate risk/high risk disease if the outcome might have an implication for adjuvant treatment strategy [IV, B].

\section{Lymph node staging}

Sentinel node biopsy has been introduced as alternative to lymph node dissection for lymph node staging, and if done according to state-of-art principles a negative sentinel node is accepted to confirm pN0. Multiple studies, including prospective cohort ones, confirmed high sensitivity of sentinel lymph node status for lymph node staging in early-stage endometrial carcinoma patients and support the impact of sentinel lymph node biopsy on surgical management and indications for adjuvant therapies ${ }^{179-241}$. More intensive pathological assessment of sentinel lymph node (sentinel lymph node ultrastaging) supports the detection of small metastases which could be missed by standard evaluation ${ }^{214,232}$. Sentinel lymph node biopsy without dissection of other pelvic lymph nodes is associated with subtantially lower risk of postoperative morbidity, especially lower leg lymphoedema ${ }^{242}$. In a large group of low risk (myometrial invasion $<50 \%$, low-grade) endometrial carcinoma patients with sentinel lymph node biopsy, lymph node involvement was found in $6 \%$ of patients, half of them identified by pathological ultrastaging 243 . Patients with tumours without myometrial invasion, did not have any positive sentinel lymph nodes. Recently, a randomised controlled trial highlighted that the use of indocyanine green instead of methylene blue dye resulted in a significant increase of sentinel lymph node detection rates per hemipelvis in women with endometrial carcinoma undergoing minimally invasive surgery ${ }^{244}$. Retrospective studies showed similar prognosis of patients after full lymphadenectomy and sentinel lymph node biopsy only ${ }^{179,201,220}$. High bilateral pelvic sentinel lymph node detection can be achieved when the tracer is injected into the cervix ${ }^{180,245}$. Higher sentinel lymph node detection rate has been reported using near-infrared fluorescence in comparison to other techniques ${ }^{246}$. A worse prognosis is associated with the presence of nodal micrometastases, especially in patients who do not receive adjuvant treatment ${ }^{247}$. There is no evidence that the presence of isolated tumour cells (ITCs) has an impact on prognosis, and similar to other 
tumour sites, the stage would be pNO(i+). If pelvic lymph node involvement is reported either by sentinel lymph node frozen section or by the final pathology, paraaortic staging can be considered, either by imaging (with all limitations of the imaging modalities) or by surgery. It should be noted that based on data from 2 large randomised trials, lymph node staging does not have a therapeutic value but is done to assess the extent of disease and to provide information for adjuvant treatment decisions ${ }^{248,249}$. Frozen section on specimen regarded as sentinel lymph nodes can confirm the presence of lymph nodes and macrometastases but should not replace adequate pathological processing and ultrastaging.

\section{Recommendations:}

- Sentinel lymph node biopsy can be considered for staging purposes in patients with low risk/intermediate risk disease. It can be omitted in cases without myometrial invasion. Systematic lymphadenectomy is not recommended in this group [II, A].

- Surgical lymph node staging should be performed in patients with high intermediate risk/high risk disease. Sentinel lymph node biopsy is an acceptable alternative to systematic lymphadenectomy for lymph node staging in stage I/II [III, B].

- If sentinel lymph node biopsy is performed [II, A]:

- Indocyanine green with cervical injection is the preferred detection technique.

- Tracer re-injection is an option if sentinel lymph node is not visualized up-front.

- Side-specific systematic lymphadenectomy should be performed in high intermediate risk/high risk patients if sentinel lymph node is not detected on either pelvic side.

- Pathological ultrastaging of sentinel lymph nodes is recommended.

- When a systematic lymphadenectomy is performed, pelvic and paraaortic infrarenal lymph node dissection is suggested [III, B].

- Presence of both macrometastases and micrometastases $(<2 \mathrm{~mm}, \mathrm{pN} 1(\mathrm{mi}))$ is regarded as a metastatic involvement [IV, C].

- The prognostic significance of ITCs, pNO(i+), is still uncertain [IV, C].

- If pelvic lymph node involvement is found intra-operatively, further systematic pelvic lymph node dissection should be omitted. However, debulking of enlarged lymph nodes and paraaortic staging can be considered [IV, B].

\section{Option for ovarian preservation and salpingectomy in stage I/II}

A meta-analysis ${ }^{250}$ showed that there was no significant difference in overall survival between the patients treated with ovarian preservation andbilateral salpingo-oophorectomy. Similar result was achieved in young and premenopausal women. Disease-free survival of patients whose ovaries were preserved was slightly compromised but this was not statistically significant. Ovarian preservation can be cautiously considered in specific clinical situations when treating young and premenopausal women with early stage endometrial carcinoma because it is not associated with a significant adverse impact on survival ${ }^{251-253}$. Salpingectomy during hysterectomy is recommended to decrease the risk of high-grade serous ovarian carcinoma ${ }^{254}$. Ovarian preservation is not recommended in patients with cancer family history involving ovarian cancer risk (e.g. BRCA mutation, Lynch syndrome, etc.), but oocyte cryopreservation might be considered $^{255}$. 


\section{Recommendations:}

- Ovarian preservation can be considered in premenopausal patients younger than 45 years old with low-grade endometrioid endometrial carcinoma with myometrial invasion $<50 \%$ and no obvious ovarian or other extra-uterine disease [IV, A].

- In cases of ovarian preservation, salpingectomy is recommended [IV, B].

- Ovarian preservation is not recommended for patients with cancer family history involving ovarian cancer risk (e.g. BRCA mutation, Lynch syndrome, etc.) [IV, B].

\section{$\underline{\text { Radicality of surgery for clinical stage II }}$}

Radicality of hysterectomy (simple versus modified radical hysterectomy (type II)) in stage I-III endometrial carcinoma has no impact on local recurrence rate, disease free survival and overall survival. In a meta-analysis enrolling 2,866 patients with stage II endometrial carcinoma, radical hysterectomy did not show a significant survival benefit for either overall survival or progression-free survival as compared to simple hysterectomy ${ }^{256}$. The result remained consistent after it was adjusted for the possible impact from adjuvant radiotherapy.

\section{Recommendations:}

- Total hysterectomy with bilateral salpingo-oophorectomy and lymph node staging is the surgical standard of care in stage II endometrial carcinoma patients [IV, B].

- More extensive procedures should only be performed if required to achieve free surgical margins [IV, B].

\section{Medically unfit patients}

It is rare for patients to be unfit for surgery, but medical co-morbidities, which increasingly include morbid obesity, can preclude surgery due to high operative and peri-operative risks. Ideally assessment should be undertaken in a centre with specialist anaesthetic experience in managing these high-risk patients. Definitive radiotherapy with brachytherapy, EBRT or the combination of both modalities can be considered ${ }^{257-261}$.

\section{Recommendations:}

- Medical contraindications to the standard surgical management by minimally invasive surgery are rare. Vaginal hysterectomy, with bilateral salpingo-oophorectomy if feasible, can be considered in patients unfit for the recommended standard surgical therapy [IV, C].

- Definitive radiotherapy can be considered for primary tumours where surgery is contraindicated for medical reasons:

- The combination of external beam radiation therapy (EBRT) and brachytherapy should be used for high-grade tumours and/or deep myometrial invasion [II, B].

- For low-grade tumours, brachytherapy alone can be considered [II, B].

- In medically unfit patients, unsuitable for curative surgery or radiotherapy, systemic treatment (including hormonal therapy) can be considered [IV, B]. 


\section{Fertility preservation}

\section{Work-up for fertility preservation treatments}

Fertility-sparing treatments should be considered in patients with atypical hyperplasia/endometrioid intraepithelial neoplasia (AH/EIN) or grade 1 endometrioid carcinoma without myometrial invasion ${ }^{262-268}$. There are very few published data ${ }^{269}$ on patients with stage IA grade 2 endometrioid carcinoma without myometrial invasion who received fertility sparing treatment with combined oral medroxyprogesterone acetate/levonorgestrel intrauterine system. Although results are encouraging, this treatment should only be considered by experienced gynaecological oncologists using well-defined protocols with detailed patient information and close follow-up.

Hysteroscopic biopsy is suggested, based on its higher agreement with final diagnosis as compared with dilatation and curettage 270,271 . Although hysteroscopy seems to be associated with a higher rate of positive peritoneal cytology, it seems not to have a negative impact on survival ${ }^{272}$. Expert vaginal ultrasound examination can be used instead of pelvic MRI. Its high diagnostic performances allows the detection of myometrial invasion and cervical stromal invasion with respect to final pathological examination. Ultrasound should be performed by an expert sonographer (a practitioner who spend a significant part of her/his time undertaking ultrasound examinations in gynecology and gynecologic oncology and has fulfilled the minimum training requirements for level 3 following the recommendations of the European Federation of Societies for Ultrasound in Medicine and Biology273).

There is currently lack of high-quality evidence regarding the correlation between weight loss and reduction of risk of recurrence/increased survival in endometrial carcinoma patients, especially with respect to fertility-sparing treatment ${ }^{274}$. Diabetes mellitus does not seem to affect the outcome of conservative treatment in women with AH/EIN or early endometrial carcinoma ${ }^{275}$. Conversely, the use of metformin seems associated with an improvement of overall survival for patients with endometrial carcinoma and reduce their risk of cancer relapse ${ }^{276}$. In addition, metformin is associated with improvement in the overall survival of endometrial carcinoma patients among diabetes.

\section{Recommendations:}

- Patients who are candidates for fertility-preserving treatment must be referred to specialised centres. Fertility-sparing treatment should be considered only in patients with AH/EIN or grade 1 endometrioid endometrial carcinoma without myometrial invasion and without genetic risk factors $[\mathrm{V}, \mathrm{A}]$.

- In these patients, endometrial biopsy, preferably through hysteroscopy, must be performed [III, A].

- AH/EIN or grade 1 endometrioid endometrial carcinoma must be confirmed/diagnosed by a pathologist experienced in gynaecological pathology [V, A].

- Radiologic imaging to assess the extension of the disease must be performed. An expert ultrasound examination can substitute pelvic MRI scan [III, B].

- Patients must be informed that fertility-sparing treatment is not a standard treatment. Only patients who strongly desire to preserve fertility should be treated conservatively. Patients must be willing to accept close follow-up and be informed of the need for future hysterectomyin case of failure of treatment and/or after pregnancies $[\mathrm{V}, \mathrm{A}]$.

\section{Management and follow-up for fertility preservation}

So far, there are no available randomized controlled trials comparing different methods of conservative treatment in women with AH/EIN or presumed stage IA grade 1 endometrioid carcinoma. Existing data ${ }^{262-}$ 
268,277-294 suggest that patients who received hysteroscopic resection followed by progestin therapy achieve the highest complete remission rate as compared with other existing fertility-preserving treatments. Intrauterine progestin therapy such as levonorgestrel-releasing intrauterine system combined with gonadotropin-release hormone receptor agonist/progestin have a satisfactory pregnancy rate and low recurrence rate. Patients who received oral progestin only might be more likely to recur and have more systemic adverse effects.

\section{$\underline{\text { Recommendations: }}$}

- All patients should be evaluated before and after the fertility-sparing treatment at a fertility clinic [IV, $\mathrm{C}]$.

- Hysteroscopic resection prior to progestin therapy can be considered [III, B].

- Medroxyprogesterone acetate (400-600 mg/day) or megestrol acetate $(160-320 \mathrm{mg} /$ day $)$ is the recommended treatment. Treatment with levonorgestrel intrauterine device in combination with oral progestins with or without gonadotropin-releasing hormone analogues can also be considered [IV, B].

- In order to assess response hysteroscopic guided biopsy and imaging at 3-4 and 6 months must be performed. If no response is achieved after 6 months, standard surgical treatment is recommended [IV, B].

- Continuous hormonal treatment should be considered in responders who wish to delay pregnancy [IV, B].

- Strict surveillance is recommended every 6 months with TVUS and physical examination. During follow-up hysteroscopic and endometrial biopsy should be performed only in case of abnormal uterine bleeding or atypical ultrasound finding [IV, B].

- Fertility-sparing treatment can be considered for intrauterine recurrences only in highly selected cases under strict surveillance [IV, C].

- Hysterectomy and bilateral salpingo-oophorectomy is recommended after childbearing due to a high recurrence rate. Preservation of the ovaries can be considered depending on age and genetic risk factors [IV, B].

\section{Synchronous presentation of low-grade endometrioid endometrial and ovarian carcinomas}

Adnexal involvement by endometrial carcinoma is currently a parameter important in FIGO staging and has an impact on overall survival rate ${ }^{295}$. It was shown that patients with simultaneous involvement of endometrium and ovary by low-grade endometrioid carcinoma had a favorable outcome. This suggested that they were synchronous primary tumours rather than metastatic sites. Several criteria have been used in the past to distinguish between endometrial carcinoma with ovarian metastasis and synchronous primary tumours ${ }^{296,297}$. However, these were not easy to apply.

Recent studies have shown that for low grade endometrioid carcinomas, there is a clonal relationship between the endometrial and ovarian carcinomas in the vast majority of cases, indicating that the carcinoma arises in the endometrium, and extends secondarily to the ovary ${ }^{298,299}$. In the most recent edition of WHO $(2020)^{33}$ it is mentioned that patients with clonally related low-grade endometrioid carcinomas be managed without adjuvant treatment (as if they were two independent primaries) when fulfilling the following criteria: 1) low-grade endometrioid morphology, 2) no more than superficial myometrial invasion, 3) absence of LVSI, and 4) absence of additional metastases ${ }^{33,300}$. 


\section{Recommendation:}

- If all WHO 2020 criteria mentioned above are met and the ovarian carcinoma is pT1a, no adjuvant treatment is recommended [III, B].

\section{ADJUVANT TREATMENT}

Adjuvant treatment recommendations for endometrial carcinoma strongly depend on the prognostic risk group. See Figure 3 for the definitions of the prognostic risk groups with and without known molecular classification.

\section{Low risk}

For patients with low-risk endometrial carcinoma, no adjuvant treatment is recommended, based on data from multiple randomized trials ${ }^{301-304}$. For patients with stage I-II POLEmut endometrial carcinomas, no adjuvant treatment seems justifiable based on the data from independent series showing very few recurrences, also in case of observation ${ }^{21,25}$. For stage III patients however, there are only indirect data to support this, as all cases with advanced disease had adjuvant treatment. In the molecular analysis of the PORTEC-3 trial of high-risk endometrial carcinoma those with POLEmut endometrioid carcinoma had an excellent outcome in both arms $^{22}$. However, both trial arms included EBRT. Prospective registration (preferably in national or international studies) of POLEmut endometrial carcinoma cases with treatment and outcome data is strongly recommended.

\section{Recommendations:}

- For patients with low-risk endometrial carcinoma, no adjuvant treatment is recommended [I, A].

- When molecular classification is known:

- For patients with endometrial carcinoma stage I-II, low-risk based on pathogenic POLE-mutation, omission of adjuvant treatment should be considered [III, A].

- For the rare patients with endometrial carcinoma stage III-IVA, and pathogenic POLE-mutation, there are no outcome data with the omission of the adjuvant treatment. Prospective registration is recommended [IV, C].

\section{Intermediate risk}

Adjuvant brachytherapy provides excellent vaginal control and high survival rates, similar to those after adjuvant EBRT in this intermediate-risk population, as shown in large randomized trials, particularly the PORTEC-2 trial and Swedish trial ${ }^{305-313}$. It was also shown that only the small minority of patients with higher risk based on substantial LVSI, p53abn or L1CAM overexpression had a slightly higher risk of pelvic recurrence with vaginal brachytherapy than those who had EBRT. Therefore, the intermediate risk category only includes those with none or only focal LVSI, and no p53abn. In a Danish population study ${ }^{314}$, it was confirmed that the risk of locoregional relapse was higher (about 14\%) with omission of vaginal brachytherapy, but that overall survival was not different due to treatment of relapse. Therefore, no adjuvant treatment is an option in this group, especially for patients below 60 years who have a lower risk of relapse.

MMRd and, especially, NSMP cancers form the majority of endometrioid carcinomas, and have an intermediate prognosis, in between POLEmut (excellent prognosis) and p53abn carcinomas (unfavourable 
prognosis). Findings of prior large randomised trials in (high-)intermediate risk endometrial carcinoma are therefore mainly applicable to MMRd and NSMP endometrioid carcinomas in this intermediate risk category.

It has to be stressed that p53abn carcinomas restricted to a polyp or without myometrial invasion were not included in the randomized trials and the value of chemotherapy and of EBRT are uncertain. Since the studies mentioned above did not include and/or not address non-endometrioid (and/or p53abn) carcinomas without myometrial invasion, there are very few specific available data on the best treatment for the stage IA non-endometrioid carcinomas (serous, clear cell, undifferentiated carcinoma, carcinosarcoma, mixed) without myometrial invasion. Some case series and a recent analysis using the U.S. National Cancer Data Base suggest that adjuvant chemotherapy (with or without vaginal brachytherapy) might improve survival, while others reports showed good outcomes with vaginal brachytherapy only ${ }^{305}$. Therefore, these carcinomas have been grouped in the intermediate risk category and adjuvant therapy should be discussed on a case by case basis until more prospective data are available.

\section{Recommendations:}

- Adjuvant brachytherapy can be recommended to decrease vaginal recurrence [I, A].

- Omission of adjuvant brachytherapy can be considered [III, C], especially for patients aged $<60$ years [II, A].

- When molecular classification is known, POLEmut and p53abn with myometrial invasion have specific recommendations (see respective recommendations for low- and high risk).

- For p53abn carcinomas restricted to a polyp or without myometrial invasion, adjuvant therapy is generally not recommended [III, C].

\section{High intermediate risk, pNO after lymph node staging}

The definition of high-intermediate risk has changed in comparison with the ESMO-ESGO-ESTRO consensus conference. In the current prognostic risk group classification (see Figure 3), stage IA endometrioid carcinomas are only included if there is substantial LVSI $^{3-5}$. This high-intermediate risk group also includes stage IB low-grade endometrioid with substantial LVSI, and stage IB high-grade endometrioid carcinomas regardless of LVSI, and stage II endometrioid carcinomas. In view of the higher risk of recurrence in this newly classified group (even with negative nodes), adjuvant brachytherapy can be recommended to decrease vaginal recurrence. In the case of substantial LVSI and/or stage II, EBRT can be considered, as it has been shown to reduce the risk of pelvic and para-aortic nodal relapse ${ }^{315}$.

In two older RCTs ${ }^{316,317}$, there was no difference between adjuvant chemotherapy alone and EBRT alone in recurrence-free and overall survival. In the NSGO/EORTC trial and the PORTEC-3 trials, the combination chemotherapy and radiotherapy seemed to provide better recurrence-free and overall survival outcomes respectively compared to radiotherapy alone ${ }^{318,319}$. The GOG-249 trial did not find benefit in recurrencefree or overall survival from 3 cycles of chemotherapy with brachytherapy compared to EBRT alone ${ }^{315}$. Molecular analysis of PORTEC-3 trial tissues suggested no benefit of chemotherapy for MMRd carcinomas ${ }^{319,320}$. Omission of adjuvant treatment is an option and this should be considered only when close follow-up is guaranteed to ensure detection and prompt treatment of recurrence at an early stage.

\section{Recommendations:}

- Adjuvant brachytherapy can be recommended to decrease vaginal recurrence [II, B].

- $\quad$ EBRT can be considered for substantial LVSI and for stage II [I, B]. 
- Adjuvant chemotherapy can be considered, especially for high-grade and/or substantial LVSI [II, C].

- Omission of any adjuvant treatment is an option [IV, C].

- When molecular classification is known, POLEmut and p53abn have specific recommendations (see respective recommendations for low- and high risk).

\section{High intermediate risk $c N 0 / p N x$ (lymph node staging not performed)}

In view of the recent randomized trials GOG-249 (for stage I and II endometrial carcinomas with high risk factors or serous or clear cell histology), the PORTEC-3 trial and the older GOG-99 trial, adjuvant EBRT is recommended in case of substantial LVSI or stage $\mathrm{II}^{301,315,318,319,321}$. Additional chemotherapy can be considered, especially for high-grade carcinomas, based on the PORTEC-3 trial, but the question remains whether the benefit outweighs the toxicity for stage I-II endometrioid carcinomas, and multidisciplinary shared decision making is needed ${ }^{319}$. Molecular analysis of PORTEC-3 trial tissues suggested no benefit of chemotherapy for MMRd carcinomas ${ }^{319,320}$. Adjuvant brachytherapy alone can be considered for LVSI negative cases and for stage II grade 1 disease.

\section{Recommendations:}

- Adjuvant EBRT is recommended, especially for substantial LVSI and/or for stage II [I, A].

- Additional adjuvant chemotherapy can be considered, especially for high-grade and/or substantial LVSI [II, B].

- Adjuvant brachytherapy alone can be considered for high-grade LVSI negative and for stage II grade 1 endometrioid carcinomas [II, B].

- When molecular classification is known, POLEmut and p53abn have specific recommendations (see respective recommendations for low- and high risk).

\section{High risk}

The risk category changes also have a substantial impact on this category. Some carcinomas designated as high-risk in the ESMO-ESGO-ESTRO consensus conference are not included anymore in the high-risk subgroup in these ESGO-ESTRO-ESP guidelines ${ }^{3-5}$. High-risk carcinomas are now either stage III-IVA without residual disease, or stage I-IVA p53abn or non-endometrioid carcinomas without residual disease with myometrial invasion (for specifics, see Figure 3).

In 2019, the updated results of the PORTEC-3 trial, with a longer median follow-up of 72 months and with $75 \%$ of participants having reached 5 years of follow-up were published ${ }^{322}$. In this trial comparing combined chemotherapy and radiotherapy $(2$ cycles of cisplatin during radiotherapy followed by 4 cycles of carboplatin-paclitaxel) with radiotherapy alone, a statistically significant $5 \%$ overall survival benefit at 5 years and a $7 \%$ failure-free survival benefit was seen in the combined therapy group as compared with radiotherapy alone. The greatest overall survival difference was seen in stage III carcinomas and in serous carcinomas regardless of stage. The GOG-258 trial compared the same chemotherapy-radiotherapy schedule used in PORTEC-3 with 6 cycles of carboplatin-paclitaxel chemotherapy alone, and found overlapping relapse-free and overall survival rates ${ }^{323}$. However, the chemotherapy alone arm had significantly higher rates of pelvic and peri-aortic nodal relapse. Therefore, chemotherapy alone is an alternative option based on the GOG-258 results for stage III-IV disease. The final analysis of the GOG-249 trial highlighted that a post-operative adjuvant strategy of vaginal cuff brachytherapy followed by 3 cycles of paclitaxel and carboplatin chemotherapy did not significantly increase 5-year recurrence-free survival or 5-year overall survival compared with pelvic radiotherapy ${ }^{324}$. Vaginal and distant recurrences rates 
were similar between arms. However, pelvic or para-aortic nodal recurrences were significantly less common with pelvic radiotherapy. The older pooled analysis of the NSGO-EORTC and MANGO-ILIADE trials used sequential chemotherapy and radiotherapy (either sequence) and reported significantly longer recurrence-free survival compared with radiotherapy alone ${ }^{318}$. Multiple retrospective studies indicated a survival benefit in advanced-stage endometrial carcinoma patients treated with post-operative combined treatment including radiotherapy and chemotherapy, delivered by either the sandwich or sequential method, compared to radiotherapy alone or chemotherapy alone ${ }^{325-343}$.

The benefit of added chemotherapy is unclear for patients with stage I-II clear cell carcinomas. These have often been included with serous as "non-endometrioid carcinomas". Of note, in the PORTEC-3 trial it was specifically in those with serous histology that a significant benefit of added chemotherapy was seen ${ }^{322}$. However this was not observed in the NSGO-EORTC and MANGO-ILEADE trials. Extended field radiotherapy is used in the case of involved para-aortic nodes or involvement of high common iliac nodes, both with or without chemotherapy. The combination of extended field radiotherapy with chemotherapy using modern intensity-modulated radiation therapy/volumetric modulated arc therapy (IMRT/VMAT) techniques has been shown feasible in the PORTEC-3 and GOG-258 trials. An additional brachytherapy boost can be considered, especially for substantial LVSI, endocervical stromal invasion and/or stage IIIBIIIC.

MMRd and NSMP carcinomas are included in the high-risk category if stage III-IVA with no residual disease. The p53abn carcinomas can be of endometrioid, serous, undifferentiated and clear cell histologic type, but all consistenty show a poor outcome and should therefore be regarded as high-risk. Based on the current data, it is more difficult to draw conclusions regarding carcinosarcomas and undifferentiated carcinomas that are NSMP endometrial carcinomas, due to lack of large series. For clear cell carcinomas, the available data suggest some prognostic information may lie in the molecular classification. About 40$50 \%$ of clear cell carcinomas are p53abn. While serous carcinomasin the PORTEC-3 trial had an unfavourable outcome and significant benefit of added adjuvant chemotherapy, those with clear cell carcinomas seemed to have an outcome similar to high-grade carcinomas in general, and were more favourable if not $\mathrm{p} 53 \mathrm{abn}^{320,322}$. The findings of the randomised trials for endometrioid carcinomas cited above are therefore largely applicable to stage III MMRd and NSMP carcinomas and to stage I-III p53abn carcinomas. This was also seen in the molecular analysis of the PORTEC-3 trial, which showed a statistically significant survival advantage for p53abn carcinomas with combined therapy, for stage I-III. In contrast, POLEmut carcinomas had almost none recurrences in both arms. There was no clear benefit of added chemotherapy for MMRd, while the NSMP carcinomas had some benefit of added chemotherapy especially in case of stage III. Prospective evaluation of the molecular characteristics in randomised trials is highly recommended.

\section{Recommendations:}

- EBRT with concurrent and adjuvant chemotherapy [I, A], or alternatively sequential chemotherapy and radiotherapy is recommended [I, B].

- Chemotherapy alone is an alternative option [I, B].

- Carcinosarcomas should be treated as high risk carcinomas (not as sarcomas) [IV, B].

- When the molecular classification is known, p53abn carcinomas without myometrial invasion and POLEmut have specific recommendations (see respective recommendations for low- and intermediate risk) [III, C]. 


\section{ADVANCED DISEASE}

\section{Surgery for clinically overt stage III and IV disease}

In stage III and IV endometrial carcinoma (including carcinosarcoma), maximal cytoreduction should be considered only if macroscopic complete resection is feasible with acceptable morbidity ${ }^{34-349}$. Surgery should be performed in a specialised centre. Pre-operative complete staging and multidisciplinary discussion within a tumour board should be performed. Suspicious enlarged lymph nodes should be resected if complete resection is possible ${ }^{350,351}$. A full systematic pelvic and para-aortic lymphadenectomy of non-suspicious lymph nodes should not be performed, because there is no evidence for a therapeutic impact. If upfront surgery is not feasible or acceptable and therefore primary systemic therapy is given, delayed surgery can be considered in case of a meaningful response to chemotherapy ${ }^{352-359}$.

\section{$\underline{\text { Recommendations: }}$}

- In stage III and IV endometrial carcinoma (including carcinosarcoma) surgical tumour debulking including enlarged lymph nodes should be considered when complete macroscopic resection is feasible with an acceptable morbidity and quality of life profile, following full pre-operative staging and discussion by a multi-disciplinary team [IV, B].

- Primary systemic therapy should be used if upfront surgery is not feasible or acceptable [IV, A].

- In cases of a good response to systemic therapy delayed surgery can be considered [IV, C].

- Only enlarged lymph nodes should be resected. Systematic lymphadenectomy is not recommended [IV, B].

\section{Unresectable primary tumour due to local extent of disease}

For patients presenting with unresectable locally advanced disease and no evidence of multiple distant metastases, treatment options include definitive radiotherapy, or neoadjuvant chemotherapy followed by surgery or definitive radiotherapy, depending on response ${ }^{260,353-355,360}$. Definitive radiotherapy comprises EBRT to the pelvis followed by image-guided brachytherapy. Concurrent chemotherapy may be considered to enhance the radiation effect. Brachytherapy should boost sites of macroscopic disease in the uterus, parametrium or vagina using the ESTRO principles. Adjuvant chemotherapy should also be considered following primary local treatment (surgery or radiotherapy) to reduce the risk of distant metastases.

\section{Recommendations:}

- For unresectable tumours, multidisciplinary team discussion should consider definitive radiotherapy with EBRT and intrauterine brachytherapy, or neoadjuvant chemotherapy prior to surgical resection or definitive radiotherapy, depending on response [IV, C].

- Image-guided brachytherapy is recommended to boost intrauterine, parametrial or vaginal disease $[\mathrm{IV}, \mathrm{A}]$.

- Chemotherapy should be considered after definitive radiotherapy [IV, B].

\section{Residual pelvic or para-aortic lymph nodes following surgery}

Residual lymph node disease can be treated with external beam radiotherapy using an integrated or sequential boost to escalate the nodal dose. An IMRT technique reduces the risk of toxicity to surrounding 
tissue $^{361}$. Adjuvant chemotherapy reduces the risk of distant metastases for patients with lymph node involvement ${ }^{319,322,323}$.

\section{Recommendations:}

- Residual lymph node disease should be treated with a combination of chemotherapy and EBRT [III, B], or chemotherapy alone [IV, B].

- $\quad$ EBRT should be delivered to pelvis and para-aortic nodes with dose escalation to involved nodes using an integrated or sequential boost [IV, B].

\section{Residual pelvic disease (positive resection margin, vaginal disease, pelvic side wall disease)}

Patients with residual pelvic disease following surgery have a high risk of both local and distant recurrence. Radiotherapy can achieve long-term local control while chemotherapy reduces the risk of distant metastases. An individualised approach with either (chemo)-radiotherapy to pelvis followed by chemotherapy or adjuvant chemotherapy followed by radiotherapy to the pelvis \pm para-aortic nodes should be considered.

\section{Recommendation:}

- An individualised approach with either radiotherapy or chemotherapy or a combination of both modalities should be considered by a multidisciplinary team $[\mathrm{V}, \mathrm{B}]$.

\section{RECURRENT DISEASE}

\section{Radiotherapy naïve patients}

Treatment of patients with recurrent endometrial carcinoma involves a multi-disciplinary approach with surgery, radiotherapy and/or systemic therapy depending on the fitness and wishes of the patient, the tumor dissemination patterns and prior treatment. A decision about surgery needs to take account of patient morbidity and wishes, available non surgical treatments and resources. The interval between primary treatment and recurrence should also be taken into consideration. Patients with recurrent disease, including resectable peritoneal and lymph node relapse, should be considered for surgery only if it is anticipated that complete resection of macroscopic disease can be achieved witha reasonable morbidity profile ${ }^{362-368}$. The extent of the operation will depend on the degree of tumour dissemination pattern.

Locoregional recurrence of endometrial carcinoma is rare. With the advent of modern image guided radiation therapy, including IMRT and image-guided adaptive brachytherapy, radiotherapy has become the treatment of choice in previously not-irradiated patients with isolated vaginal recurrence or locoregional recurrence ${ }^{362,363,369-378}$. Consideration should be given to remove solitary, easily accessible vaginal relapses, for better local symptom control prior to radiotherapy.

\section{Recommendations:}

- Patients with recurrent disease (including peritoneal and lymph node relapse) should be considered for surgery only if it is anticipated that complete removal of macroscopic disease can be achieved with acceptable morbidity. Systemic and/orradiation therapy should be considered postoperatively depending on the extent and pattern of relapse, and the amount of residual disease [IV, C].

- In selected cases, palliative surgery can be performed to alleviate symptoms (e.g. bleeding, fistula, bowel obstruction) [IV, B]. 
- For locoregional recurrence, the preferred primary therapy should be EBRT \pm chemotherapy with brachytherapy [IV, A].

- An easily accessable, superficial vaginal tumour can be resected vaginally prior to radiotherapy [IV, C].

- For vaginal cuff recurrence:

- Pelvic EBRT + intracavitary ( \pm interstitial) image guided brachytherapy is recommended [IV, A]

- In case of superficial tumours: Intracavitary brachytherapy alone can be considered [IV, A]

- $\quad$ Systemic treatment can be considered before or after radiotherapy [IV, C].

\section{Radiotherapy pretreated patients with locoregional recurrence}

In patients who have previously received EBRT \pm brachytherapy, radical surgery with the intention of complete resection with clear margins should be considered in specialized centresafter ruling out metastatic disease with modern imaging. Pelvic exenteration may be considered for central local relapse $^{348,379,380}$. Otherwise, further radiation should be considered as radical therapy with or without systemic therapy. Interstitial brachytherapy (low-dose rate or high-dose rate) as sole modality of treatment or combined with EBRT can result in high local control over 1-5 years ${ }^{373,374,381,382}$. Other techniques like permanent seed implant or post-operative electron irradiation, protons and stereotactic body radiotherapy may be reccommended in highly selected patients ${ }^{383-385}$. The appropriate dose for each case needs to be individualized. Some low dose rate data suggests improved outcomes with doses $>50 \mathrm{~Gy}$. The high-dose rate data are more varied, suggesting an improved local control with doses $>40 \mathrm{~Gy}$. In general, a longer time interval between the first and second course of radiation as well as recurrences $<2$ $4 \mathrm{~cm}$ tend to have improved outcomes. Multidisciplinary management is critical to develop individualized plans and to clearly communicate potential side effects and expected treatment outcomes.

\section{Recommendations:}

- In patients with a history of previous radiation, radical surgery, including exenteration, should be considered when the intention is complete resection with clear margins [IV, B].

- Additional options to consider include intraoperative electron radiation therapy or other forms of radiation therapy [IV, C].

- If surgery is not feasible, radical re-irradiation options include stereotactic body radiotherapy targeting the recurrence, permanent seed implants or proton therapy. In selected cases, limited volume re-irradiation with EBRT and brachytherapy boost may be an option (especially if longer interval from the first irradiation) [IV, C].

- In patients who only had previous brachytherapy, EBRT + brachytherapy boost is recommended [IV, C].

- In patients where re-irradiation with ERBT is not an option, image-guided interstitial brachytherapy only is recommended (may improve outcome) [IV, C].

\section{Oligometastatic recurrent disease}

Oligometastases is a disease concept that is defined by a state of limited metastatic tumours for which local ablative therapy could be curative. It refers in general to cancer patients with 1 to 5 metastases or recurrences ${ }^{386-388}$. In recent years, the concept of oligometastatic relapse has evolved and has led to a change in the approach to treatment. A prolonged disease-free interval and perhaps even cure may be achieve in some situations where the primary cancer site (if still present) is controlled, and metastatic 
sites are ablated (surgically or with radiation) ${ }^{389-392}$. Multi-disciplinary management is critical to develop individualized plans and to communicate potential side effects and expected treatment outcomes. The additional benefit of chemotherapy is uncertain.

\section{Recommendations:}

- Patients with oligometastatic disease should be considered for radical local therapy [IV, B].

- Treatment options include [IV, B]:

- Surgery

- Radiation therapy including stereotactic radiotherapy

- Local ablating techniques

- The additional benefit of chemotherapy is uncertain [IV, B].

\section{Systemic treatment for recurrent disease}

Hormonal treatment results in a response rate of up to $55 \%$ in advanced/recurrent endometrial carcinoma ${ }^{393}$. Low-grade, slowly progressing, hormone receptor positive tumours appear to gain the greatest benefit from treatment, however, clinical benefit has also been observed in patients with hormone receptor negative tumours ${ }^{394}$. Progestogens are generally recommended ${ }^{394}$. Alternative options include aromatases inhibitors, tamoxifen and fulvestrant. In the PARAGONtrial a response rate of $7 \%$ and a clinical benefit rate of $44 \%$ was reported with anastrazole in a cohort of 82 patients with recurrent, receptor positive, endometrial carcinoma ${ }^{395}$. A single-arm phase II trial demonstrated a high response rate and clinical benefit rate with the combination of letrozole and everolimus ${ }^{396}$. Confirmation of hormone receptor status by biopsy should be considered at the time of recurrence because of a potential change in hormone receptor expression betweenprimary tumour and recurrence. In patients undergoing hormonal therapy, the risk of thrombo-embolic events needs to be taken into account. Prophylaxis with low molecular weight heparin should be considered in patients at high risk for thrombosis, and be given according to local guidelines. There are no universally agreed recommendations to predict a response to hormonal therapy in endometrial carcinoma, based on oestrogen and progesterone receptor immunohistochemical status. Some of the following should be taken into account: 1) a wide range of hormonal agents are used, including medroxyprogesterone acetate and synthetic progestational agents, luteinizing hormone releasing hormone antagonists, tamoxifen, and new generations of selective estrogen receptor modulators; each has a different molecular action and may therefore have different activity; 2) receptor-negative status is not an absolute contraindication to hormone treatment; 3) in some reports, response rates to various hormonal treatments for endometrial carcinoma patients are higher for patients with progesterone receptor expression; 4) the methodology for assessing and scoring hormone receptor expression in endometrial carcinoma is variable in the reported series; 5) assessment of oestrogen and progesterone receptor status in the primary tumor may not reflect the status in the recurrent or metastatic tumour and thus a biopsy of recurrent or metastatic carcinomas for hormone receptor analysis may be helpful; 6) from a pragmatic viewpoint, it seems reasonable to interpret a carcinoma as receptor positive when immunoreactivity for oestrogen receptor or progesterone receptors isfound in more than $1 \%$ of carcinoma cells, until stronger validated scientific evidence is provided.

The combination of carboplatin and paclitaxel is the standard chemotherapy treatment of advanced/recurrent endometrial carcinoma based on a randomized phase 3 trial comparing carboplatinpaclitaxel versus carboplatin-paclitaxel-anthracyclines that reported overlapping progression-free survival and overall survival between the two arms but an incresed toxicity for the triplet combination ${ }^{397}$. No standard treatment has been identified as second line therapy; a response rate of about $10-15 \%$ has 
been seen among all the available treatment options. Thus, enrollment of patients in clinical trials is strongly encouraged. Weekly paclitaxel and anthracyclines (including pegylated liposomal doxorubicin when available) are considered to be active drugs. The reintroduction of carboplatin may be considered after a prolonged interval from the last platinum treatment, based on the results of a single-centre retrospective series in patients treated with a median platinum-free interval of 25 (8-79) months. A response rate of 50\%, and median progression-free and median overall survival of 10 and 27 months, respectively, was reported after platinum re-challenge ${ }^{398}$.

Several anti PD-1 and anti PD-L1 checkpoint inhibitors have been shown to have activity in endometrial carcinoma and thus far pembrolizumab has been approved by the Food and Drug Administration (FDA), based on the results of a phase 2 single arm trial-, for the treatment of MSI-high (MSI-H)/MMRd solid tumours, that have progressed on conventional therapy ${ }^{399,400}$. The combination of intravenous pembrolizumab and lenvatinib, an oral multi-receptor tyrosine kinase inhibitor received FDA approval in October 2019 for the second-line systemic therapy of microsatellite-stable (MSS) (ie non-MSI-H/MMRd) endometrial carcinoma, based on the results of a phase 2 single-arm trial reporting $36 \%$ response rate in this population, including significant activity in those with serous carcinoma ${ }^{401,402}$. No Phase III randomized data are yet available.

Approximately 30\% of uterine serous carcinomas show HER2/neu over expression. A small randomized phase II trial of paclitaxel and carboplatin with or without trastuzumab in HER2/neu positive disease showed a 4.6 month increase in median progression-free survival ${ }^{403}$. Anti-angiogenic agents and PI3kinase/mTor and MEK inhibitors also have demonstrate activity but secure evidence of benefit is inconclusive due to the limited sample size of the trials, inconsistency of results and low therapeutic index of the drugs, suggesting further investigations in well designed and properly powered molecularly driven randomized trials are warranted ${ }^{404-415}$.

\section{$\underline{\text { Recommendations: }}$}

- Hormone therapy is the preferred front-line systemic therapy for patients with low-grade carcinomas without rapidly progressive disease [II, A].

- Progestogens (medroxyprogesterone acetate 200 (-300) $\mathrm{mg}$ and megestrol acetate $160 \mathrm{mg})$ are recommended [III, A].

- Alternative options for hormonal therapies include aromatases inhibitors, tamoxifen, fulvestrant [III, C].

- The standard chemotherapy treatment is carboplatin AUC 5-6 + paclitaxel $175 \mathrm{mg} / \mathrm{m}^{2} \mathrm{~d} 1 \mathrm{q} 21$ for 6 cycles [I, A].

- There is no standard of care for second line chemotherapy. Doxorubicin and paclitaxel are considered the most active therapies [IV, C].

- In patients with a long platinum-free interval, re-introduction of platinum can be considered [IV, C].

- Anti-PD1 based immune therapy with pembrolizumab could be considered for second line therapy of MSI/MMRd carcinomas. The combination of pembrolizumab and themulti-tyrosine-kinase inhibitor lenvatinib could be considered for second-line treatment of MSS carcinomas [III, B]. But its use may be limited due to regulatory approvals or reimbursement in different countries. Clinical trial participation should be offered to all patients with relapse disease [V, B]. 


\section{Palliative radiotherapy}

Historically radiotherapy has been an efficient treatment to palliate bleeding and pain from pelvic disease or systemic metastases. This results in rapid pain relief and temporary cessation of bleeding in the majority of patients ${ }^{416}$.

\section{Recommendations:}

- Radiotherapy is indicated for palliation of symptoms related to pelvic or systemic disease [IV, A].

- Hypofractionated small volume EBRT can be used for treating primary disease in patients not fit for radical treatment [IV, B].

\section{PRINCIPLES OF RADIOTHERAPY}

The following sections present the general principles, the principles of adjuvant radiotherapy, of definitive treatment, and of radiotherapy for recurrent disease $257-260,306,361,371,376,417-422$.

\section{General principles}

State of art techniques and radiotherapy dose are chosen based on clinical findings, pathology and patient factors including co-morbidities. For complex treatments or rare cases, referral to a specialized centre is recommended. Prospective assessment of toxicity is recommended. Patients should have counselling on pelvic care and general and sexual rehabilitation whenever appropriate.

\section{Adjuvant radiotherapy}

Radiotherapy should preferably commence within $6(-8)$ weeks of surgery, or be scheduled in relation to chemotherapy.

\section{$\underline{\text { EBRT }}$}

IMRT/VMAT techniques are recommended because the more conformal dose distribution increases normal tissue sparing compared to a four-field conventional or 3D-conformal plan ${ }^{423}$. The clinical target volume (CTV) includes the pelvic nodes (external iliac, internal iliac, obturator, distal common iliac), parametria and upper vagina. The upper common iliac and sub-aortic presacral lymph nodes are included when there is cervical stromal involvement and/or pelvic lymph node involvement. The lymph node target volume may be extended to include the aortic bifurcation or para-aortic nodes, up to or just above the level of the renal vessels, depending on the location and number of positive lymph nodes, site of sentinel lymph nodes and whether there is extra-uterine primary tumour involvement. The clinical target volume should be individualised when there is a positive resection margin, pelvic peritoneal disease or vaginal involvement. Treatment with a comfortably full bladder reduces the volume of irradiated small bowel and bladder. The planning target volume (PTV) should account of potential internal motion, depending on the method of verification used during the course of treatment. Image guided radiotherapy (IGRT) by repeated volumetric imaging with cone beam CT (and use of so-called library of plans or plan of the day techniques) mayenable the use of smaller CTV-PTV margins to reduce normal tissue toxicity. The prescription dose is commonly 45-50.4 Gy in 25-28 fractions over 5-6 weeks. An integrated or sequential EBRT boost is given to residual lymph node disease, sites of extracapsular nodal spread and positive lateral resection margins with a total dose of 55-60 Gy EQD2 ${ }_{10}$ for microscopic residual disease, or up to 66 Gy for macroscopic/bulky disease. Concurrent and adjuvant chemotherapy may be considered for stage III disease, serous histology and/or recurrent disease. 


\section{Vaginal brachytherapy}

Vaginal examination is undertaken to ensure the vaginal cuff is healed, and to assess the size and shape of vagina to guide applicator selection. Usually a vaginal cylinder is used but other applicators can be used, depending on patient anatomy. The target volume is individually determined and is usually the upper third of the vagina to a depth of $5 \mathrm{~mm}$ (both superiorly and halfway along the active length). The highdose rate brachytherapy dose is most commonly 21-24 Gy in 3 - 4 fractions to $0.5 \mathrm{~cm}$ from the applicator surface, or 8-11 Gy in 2-3 fractions when given as a boost following EBRT. A higher dose is required for treatment of residual disease or positive margins. Pulsed-dose rate brachytherapy can be used following EBRT to boost macroscopic residual disease witha dose of 15-25Gy. The treatment planning options are to use a standard library plan for each applicator size and treatment length orto use image-guided adaptive brachytherapy. In institutions where image-guided adaptive brachytherapy is applied, imaging of the applicator with CT scan or MRI evaluates whether the applicator is in close apposition to the vaginal mucosa and dose to organs at risk. This allows verification and calculation of cumulative doses, especially if vaginal brachytherapy is used as a boost after EBRT. Image-guided adaptive brachytherapy is strongly recommended when there is residual vaginal disease following surgery using similar principles to treatment for recurrent disease.

\section{Definitive treatment}

Definitive radiotherapy with EBRT, brachytherapy or a combination of both is indicated for primary tumours where surgery is contra-indicated for medical reasons. If patients are medically unfit for surgery, consider whether a long course of EBRT would be tolerated or if not, a more hypofractionated approach could be used. Intrauterine brachytherapy as a sole treatment modality is used for low-grade, early stage disease whereas the combination of EBRT and intracavitary brachytherapy is recommended for highgrade tumours and/or deep myometrial invasion. Specialist anaesthetic review may be required to assess suitability for brachytherapy, or whether brachytherapy could be applied with local anaesthesia only. More advanced inoperable disease is treated with a combination of pelvic EBRT and intrauterine brachytherapy with or without concurrent platinum-based chemotherapy. External beam radiotherapy is planned with at least 3-dimensional (3D) conformal radiotherapy to ensure inclusion of the whole uterus. The preferred technique is intensity-modulated radiotherapy with adaptive image guidance to verify target volume coverage and to maximise normal tissue sparing. A highly conformal EBRT boost (with IMRT or stereotactic body radiotherapy) can be used to escalate the total dose to the tumour site in the uterus to at least 65Gy if brachytherapy is not feasible.

Image-guided adaptive brachytherapy is recommended, preferably using MRI at the time of brachytherapy, in order to optimize tumour coverage and organ at risk doses. The brachytherapy applicator should consist of an intrauterine applicator (preferably a dedicated applicator with multiple channels for the larger uterus) and a vaginal component depending on the extent of any extra-uterine disease. Interstitial applications may be required to achieve adequate coverage. In view of the rarity of definitive treatment for endometrial carcinoma, referral to a dedicated centre is recommended. The tumour-related target volumes include the (residual) gross tumor volume on MRI (GTV-res) and the CTV is the whole uterus and any extra-uterine sites of extension before EBRT. The treatment plan aims include a total dose (EQD2 10 ) of at least 80 Gy to GTV-res, CTV D90 of about 48 Gy with brachytherapy alone and 60-65 Gy with the combination of EBRT and brachytherapy.

\section{Recurrent disease}

Radiotherapy treatmentfor recurrent endometrial carcinoma depends on the site of disease and any previous treatment. It involves EBRT, brachytherapy or a combination of both modalities. Concurrent or sequential chemotherapy may also be considered. 


\section{$\underline{\text { Radiation naive or previous brachytherapy only }}$}

Pelvic EBRT is used according to the guidelines above. Brachytherapy is used to boost recurrent disease in the vagina; in selected cases with superficial tumors brachytherapy alone can be considered. The brachytherapy applicator options include a vaginal cylinder or mould for superficial lesions whereas interstitial applicators can be used for bulkier tumours.

Image-guided adaptive brachytherapy is recommended, preferably using MRI at the time of brachytherapy, in order to optimize tumour coverage and organ at risk doses. When image-guided adaptive brachytherapyis used, the target volumes should be contoured according to the recent GECESTRO recommendation for primary vaginal cancer, aiming for a total dose (EQD2 ${ }_{10}$ ) of 80-85 Gy to CTV D90 with the combination of EBRT and image-guided brachytherapy ${ }^{421}$. If brachytherapy is not feasible due to tumour location or topography, a sequential EBRT boost with conformal radiotherapy, IMRT or stereotactic body radiotherapy is used to deliver a total GTV dose ofat least 65 Gy EQD2 ${ }_{10}$.

\section{$\underline{\text { Re-irradiation }}$}

Re-irradiation is individualised according to the extent of disease, previous radiation fields and time elapsed from the previous treatment. In general, recurrences with a longer disease free interval as well as recurrences less than 2-4 cm tend to have improved outcomes. Ideally this should be done in specialist centres with prospective collection of dosimetric and clinical data. The most common re-irradiation technique is intracavitary-interstitial brachytherapy, preferably image-guided with CT scan or MRI ${ }^{420}$. However in selected cases EBRT, stereotactic body radiotherapy, proton or carbon ion therapy is an option, particularly for pelvic sidewall or lymph node disease. Organ at risk dose constraints should take into account prior radiotherapy treatment to derive cumulative doses. Some low-dose rate data suggests improved outcomes with doses more than $50 \mathrm{~Gy}$. The high-dose rate data is more varied with some studies suggesting improved local control with doses more than 40 Gy EQD2 $2_{10}$.

\section{PRINCIPLES OF PATHOLOGICAL EVALUATION}

The following sections present the requirements for specimen submitted for pathological evaluation including specimen grossing and sampling, for the pathology report, and the molecular classification $^{19,21,23,26,424,425}$. The following sections are proposed in agreement with the recently published recommendations from the ISGyP and International Collaboration on Cancer Reporting, and WHO Classification of tumours ( $5^{\text {th }}$ edition $)^{9,33,426-428}$.

\section{Requirements for specimen submitted for pathological evaluation}

Patient information, previous cytology, histological specimens, clinical and radiological data, need to be included on the specimen request form, particularly if there is no electronic patient file. This needs to provide itemised details of biopsy, and surgical specimen (type of hysterectomy, presence of ovaries and fallopian tubes, presence of lymph nodes and designation of the lymph node sites). Biopsies should be sent to the pathology department in a container with liquid fixative (10\% neutral formalin is preferred). Surgical specimens should be either sent in a fixative or preferably fresh if there is a specific workflow for it and if the microbiological risk is controlled. This allows proper opening of the uterus and sampling a fresh tissue for research purposes.

\section{Specimen grossing and sampling}

All pathology reports should include a detailed section, code/block key on which the origin/designation of all tissue blocks should be recorded. 
The specimen needs to be oriented, that means that the anterior and posterior walls of the uterus are identified using anatomic landmarks such as the peritoneal reflection and the round ligament/ovaries. Document all organs/structures received and record their measurements and gross appearance.

The uterus should be opened immediately upon receipt in the pathology laboratory and placed in formalin within an hour of opening whenever possible. If the uterus is not immediately sent to a pathology laboratory the uterine cavity needs to be opened technically correct to guarantee proper fixation. The uterus is preferably opened along the lateral uterine walls ( 3 and 9 o'clock), although 12 and 6 o'clock sectioning may be acceptable

The pathology laboratory personnel and/or pathologists should manage the requests for fresh tissue for banking and/or investigational protocols and this task should be completed as soon as the specimen is received in the pathology laboratory.

Inking of peritoneal and/or non-peritoneal surfaces is recommended in hysterectomy specimens and is mandatory in radical hysterectomy specimens in which parametrium and vaginal cuff are present.

At least the largest dimension of the tumour must be provided, although providing 3 dimensions is recommended.

Horizontal/transverse sectioning is recommended.

Sampling one section per centimetre of the largest tumour dimension is recommended.

In case of preoperative endometrial sampling with a malignant diagnosis and no visible lesion on gross examination or a history of atypical endometrial hyperplasia/EIN, the entire endometrium and adjacent inner myometrium should be submitted for microscopic examination. The same applies to hysterectomy specimens that have been obtained for other reasons (leiomyomas, adenomyosis, etc.) when the endometrium is grossly inconspicuous but endometrial carcinoma or atypical endometrial hyperplasia/EIN are detected on the initial histological sections.

At least, one full thickness section of the uterine wall-including serosa, is required to show the deepest point of myometrial invasion.

The number of sections submitted should not be altered in the context of adenomyosis. However, in cases where the assessment of myometrial invasion is difficult because of tumour involving adenomyosis taking additional sections of the uterine wall may be useful.

Whenever possible, the interface between the tumour and its surrounding should be submitted for microscopic examination. This facilitates the measurement of the depth of myometrial invasion and the identification of precursor lesions.

At least one representative section of non-neoplastic endometrium should be submitted for microscopic examination. In addition, any grossly identified endometrial lesions separate from the tumour should be submitted.

All gross endometrial abnormalities need to be submitted for microscopic examination in hysterectomy specimen from Lynch syndrome patients. In the absence of a gross lesion, the endometrium should be submitted in toto, including the lower uterine segment.

A minimum of 2 sections ( 1 anterior, 1 posterior) should be submitted from the lower uterine segment.

Parametrial tissue/parametrium should be sampled before opening the uterus as this approach minimizes the chance of finding carryovers. All of the parametrial tissue/parametrium should be submitted for 
histologic examination. If macroscopic tumour is seen in the parametrial tissue/parametrium, the most proximal parametrial section should include the adjacent outer portion of the cervical wall.

The cervix should be left attached to the corpus during the gross examination of a hysterectomy specimen obtained for endometrial carcinoma. At least 2 full thickness sections ( 1 anterior and 1 posterior) should be submitted from a grossly unremarkable cervix. At least 2 representative sections of tumour involving the cervix should be submitted when cervix is grossly involved by endometrial carcinoma. These sections must include the full thickness of the cervical wall and the ectocervical or vaginal cuff margin.

Gross examination of a morcellated hysterectomy specimen requires special attention to identify any endometrial abnormality, although this may be extremely difficult to see in some cases. If such an abnormality is detected, the entire endometrial lesion and the adjacent myometrium should be submitted for microscopic examination. In addition, sampling of myometrial tissue containing any serosal surface should be undertaken. If the endometrium appears grossly unremarkable and the initial representative sections demonstrate the presence of atypical endometrial hyperplasia/EIN or endometrial carcinoma, careful re-grossing is required with the submission of all the visible endometrial lining and adjacent myometrium. If the morcellated specimen contains the uterine cervix, this should be sampled representatively.

Gross examination of the fallopian tube must be carefully undertaken and any areas with macroscopic abnormalities should be submitted for microscopic examination. If the fallopian tube is unremarkable, the entire tube should be submitted for microscopic examination using the sectioning and extensively examining the fimbriated end (according to the SEE-FIM protocol), particularly for serous carcinoma, and carcinosarcoma, while only the fimbrial end should be submitted in toto in other scenarios-using the guidelines of the SEE-FIM protocol, along representative cross-sections of the remainder of the fallopian tube.

Gross examination of the ovary must be carefully performed. In case of endometrial serous, clear cell carcinoma or carcinosarcoma, the entire ovary should be submitted after slicing it perpendicularly to its long axis at 2 to $3 \mathrm{~mm}$ intervals. If possible, the same protocol should be used for oophorectomy specimens accompanying hysterectomies for other endometrial carcinoma histotypes. Should the latter not be possible, at least 2 sections of each ovary should be submitted.

Omentectomy is part of the staging procedure of endometrial serous carcinoma, undifferentiated carcinoma and carcinosarcoma. The gross appearance and measurement of the omentum should be provided. Omental tissue should be sliced at $0.5 \mathrm{~cm}$ intervals to detect small abnormalities. If the omentum is grossly positive, one or 2 representative sections are enough for microscopic evaluation, but if it is grossly negative, one representative section per 2 or $3 \mathrm{~cm}$ of maximal omental dimension or at least a total of 4 blocks of tissue should be submitted.

Lymph nodes from different anatomical sites should be sent in separate appropriately labelled specimen containers and handled separately. They should be carefully dissected from the adipose tissue. This can be done with a thorough visual examination and palpation. A small amount of adipose tissue should be left around larger lymph nodes to evaluate the presence or absence of extranodal extension. Lymph nodes up to $2 \mathrm{~mm}$ are totally embedded. If larger than $2 \mathrm{~mm}$, parallel slices at 2 to $3 \mathrm{~mm}$ intervals perpendicular to the long axis of the node should be performed. All grossly unremarkable lymph node tissue should be submitted for microscopic examination. The number of lymph nodes submitted per cassette and the way they have been submitted, for example in toto - if very small, or sectioned, should be specified in the section code. With grossly positive lymph nodes, representative sections to demonstrate the largest size of tumour involvement as well as the surrounding adipose tissue should be submitted for microscopic examination and noted in the section code. 
The description of the sentinel lymph node should include gross measurement and description of gross appearance including the presence of dye. The lymph node is sliced at $2-3 \mathrm{~mm}$ intervals perpendicular to its long axis. A small rim of adipose tissue should be left around the lymph node. The entire lymph node is submitted for microscopic examination in properly coded cassettes. Ultrastaging is encouraged (i.e. additional recuts and/or IHC for keratin). At the present time there is no universal ultrastaging protocol.

Frozen section for intraoperative assessment is not encouraged for myometrial invasion assessment because of poor reproducibility and because it interferes with pre-analytical issues and possibility of carryovers.

\section{Report of pathology results (required items)}

- Description of the specimen(s) submitted for histological evaluation

- Attached anatomical structures

- Accompanying specimens

- $\quad$ Tumour type(WHO Classification of tumours ( $5^{\text {th }}$ edition $)$ )

- $\quad$ Tumour grade (FIGO, and WHO Classification of tumours ( $5^{\text {th }}$ edition)). Endometrioid endometrial carcinoma is graded using FIGO grading criteria: grades 1, 2 and 3 tumours exhibit $\leq 5 \%, 6-50 \%$ and $>50 \%$ solid non-glandular (including cribriform), non-squamous growth. The presence of severe cytologicatypia in the majority of cells ( $>50 \%$ ) increases the grade by one level, but serous carcinoma should be excluded in cases with nuclear atypia that is out of proportion to the architecture. Binary grading is recommended by the WHO Classification of tumours ( $5^{\text {th }}$ Edition), whereby grades $1-2$ tumours are classified as low-grade and grade 3 tumours as high-grade.

- Absence or presence and depth of myometrial invasion should be reported in all endometrial carcinoma as "none or less than half" OR "half or more." The measurement should be performed from the adjacent endometrial-myometrial interface.

- If myometrial invasion occurs from carcinoma within adenomyosis, the deepest myoinvasive point should be reported according to where this is located in the myometrium, and regardless of whether or not it arises from adenomyosis. In case of an exophytic tumour, the depth of myometrial invasion, and not tumour thickness, should be measured by identifying the adjacent endomyometrial junction and by correlating with the macroscopic appearance. For tumours involving polyps, measurement of invasion is performed only if the tumour invades the underlying myometrium and measurement.

- LVSI should be unequivocal, and reported as focal and extensive/substantial (5 vessels or more). LVSI should not be included in assessment of myometrial invasion depth.

- Cervical stromal invasion: for the purposes of standard reporting, the uppermost endocervical mucinous gland identified in the section should be taken as the upper limit of the endocervix.

- Vaginal involvement.

- Uterine serosal involvement. Tumour infiltrating the full myometrial thickness and reaching submesothelial fibro-connective tissue or the mesothelial layer should be reported as serosal involvement; tumour may or may not be present on the surface of the uterus; a desmoplastic response may or may not be present.

- $\quad$ Parametrial involvement. 
- Adnexal involvement. Care should be taken to determine whether the ovarian involvement is considered to be metastatic or "synchronous". Synchronous low-grade endometrioid carcinomas of the endometrium and the ovary have been demonstrated mostly to be clonally related in the vast majority of cases. Their reported indolent behaviour supports conservative management when the following criteria are met: (a) both tumours are low-grade; (b) $<50 \%$ myometrial invasion; (c) no involvement of any other site, (d) absence of extensive LVSI at any location. These parameters should be reported and included in a specific comment.

In cases of serous endometrial carcinoma with coexisting tubal intraepithelial (mucosal) carcinoma, with or without stromal invasion, ancillary techniques should be undertaken to help define whether the Fallopian lesion is independent or metastatic. In cases of endometrioid endometrial carcinoma, a comment may be included on the unknown prognostic significance of this finding.

- Omental involvement.

- Peritoneal involvement.

- Lymph node status including sentinel lymph node status reportsthe total number of nodes found and the number of positive lymph nodes, and the presence of extranodal extension (list for all separates sites). Micrometastasis ( $>0.2 \mathrm{~mm}$ and up to $2 \mathrm{~mm}$ ) are reported as pN1(mi). ITCs no greater than 0.2 $\mathrm{mm}$ in regional nodes should be reported as pN0 (i+).

- Pathologically proven distant metastases.

- $\quad$ Required ancillary techniques (IHC for p53, MSH-6 and PMS-2, complemented with MLH-1 and MSH2, MLH-1 promoter methylation analysis in cases of MLH-1/PMS-2 decrease expression). Additional immunohistochemical markers may be important for pathological diagnosis (PTEN, p16, ER, Napsin A, Racemase, Pax8, E-Cadherin) or prognosis (L1CAM).

- Provisional pathological staging pretumour board/multidisciplinary team meeting. The TNM staging system (Union for International Cancer Control and American Joint Committee on Cancer versions) for endometrioid carcinoma is largely concordant with the widely used FIGO System.

\section{Report of pathology results (recommended items unrelated to stage and with limited supporting evidence)}

- Tumour site.

- Tumour size.

- Percentages of different components of mixed carcinoma and in carcinosarcoma.

- Measurement of absolute depth of myometrial invasion, percentage of myometrium infiltrated by tumour, invasion of inner, middle, or outer one third of the myometrium, distance of myoinvasive tumour to serosal surface.

- Microcystic, elongated, fragmented pattern of invasion.

- Peritoneal cytology (if available).

- Recommended ancillary investigations. 


\section{Molecular classification}

Decision to use molecular classification in all endometrial carcinoma cases, in the subset of high-grade, or high-risk tumours, or in none of the cases, depends on the availability of resources, and decision by the multidisciplinary team of each centre.

Molecular classification is recommended to be performed by the TCGA-surrogate using the diagnostic algorithm provided by Vermij et al. ${ }^{24}$. This diagnostic algorithm requires testing of three immunohistochemical markers (p53, MSH-6, PMS-2) and somatic mutation analysis of POLE (exons 9, 11, $13,14)$. Guidance on the interpretation of pathogenicity of POLE variants is provided by Leon-Castillo et $a l^{26}$.

Five categories of tumours are recognized: 1) Ultramutated/with pathogenic POLE mutations; 2) Hypermutated with MSI/MMRd (loss of MMR protein immunoreactivity); 3) High copy number/p53abn (p53 mutant immunoreactive pattern), 4) Low copy number/NSMP (retained MMR protein immunoreactivity, and p53 wild-type immunoreactive pattern); 5) Multiple classifier (any combination of markers included in the previous categories).

If available, molecular classification data should be integrated into conventional pathologic diagnosis. Report should include information regarding the methods used for IHC as well as for POLE mutation analysis. Report should include information from the literature, regarding the pathogenicity of each POLE mutation detected ${ }^{26}$.

\section{PSYCHO-ONCOLOGICAL SUPPORT FOR WOMEN WITH ENDOMETRIAL CARCINOMA}

Endometrial carcinoma, even as a cancer with a relatively good prognosis, is a life-threatening disease. Treatment may produce significant toxicities which cause substantial short- and long-term side effects, functional loss in various behavioral and life domains as well as psychosocial distress. The patient and her caregivers may facemajor challenges in terms of coping and adjustment.

Therefore, continuous evaluation for psychological distress, sexual dysfunction and psychiatric comorbidity as well as identification of psychosocial needs are of major importance ${ }^{429}$. The first step includes an early assessment and identification of the patient's distress ${ }^{430}$. There are several standardized and validated screening instruments available like the Hospital Anxiety and Depression Scale or the easy to use Distress Thermometer ${ }^{431}$. Depending on the result of the diagnostic process various interventions should be offered such as counselling, individual or group psychotherapy, psychoeducational interventions, art therapies or relaxation techniques. For patients with a disease involving genital organs, cancer itself, surgical treatment and subsequent hormonal loss may impair sexual function. Therefore, discussion and treatment of sexual problems should be integrated as part of a holistic approach.

In order to empower patients to cope with physical and psychosocial long-term side effects of disease, treatment and to preserve quality of life, they should receive a personalized survivorship care plan including information and education to life style and prevention of secondary malignancies and other diseases. Contact to advocacy groups should be offered to all patients.

\section{AKNOWLEDGEMENTS}

The authors thank ESGO, ESTRO, and ESP for their support. ESGO office, especially Kamila Macku, provided invaluable logistical and administrative support throughout the process. The authors also thank the 191 international reviewers (physicians and patient representatives, Appendix 2) for their valuable comments and suggestions. The European Society for Medical Oncology, Professor Cristiana Sessa and the 
ESMO-ESGO-ESTRO consensus conference working group are gratefully acknowledged for the previous 2014 Endometrial Consensus Conference. The authors wish to express sincere gratitude to Annette Hasenburg and Joachim Weis for describing the psycho-oncological aspects in this article.

\section{FUNDING}

All costs relating to the development process were covered from ESGO, ESTRO, and ESP funds. There was no external funding of the development process or manuscript production.

\section{DISCLOSURE}

NCon has reported advisory boards for Seattle Genetics, AstraZeneca and Mersana, education fees from Medscape Oncology, and grants for travelling from Roche, Genmab and Amgen; IV has reported advisory boards for Amgen, AstraZeneca, Clovis Oncology, Carrick Therapeutics, Debiopharm International, F. Hoffmann-La Roche, Genmab, GSK, Immunogen, Millenium Pharmaceuticals, MSD Belgium, Octimet Oncology, Oncoinvent, Pharmamar-Doctaforum Servicios, Roche, Sotio a.s., Tesaro, Deciphera Pharmaceuticals and Verastem Oncology (fees for consulting to his university), contracted research (KU Leuven) for Oncoinvent AS and Genmab, corporate sponsored research for Amgen and Roche, and grants for travelling from Amgen, MSD/Merck, Roche, AstraZeneca and Tesaro; DC has reported advisory boards for AstraZeneca, Roche, Sotio and Novocure; MRM has reported personal financial interests for AstraZeneca, Biocard, Clovis Oncology, Geneos, Genmab, Karyopharm Therapeutics, Merck, Mersana, MSD, Oncology Venture, Pfizer, Roche, SeatleGenetics, SeraPrognostics, Sotio, Tesaro-GSK, ZaiLab, leadership role for Karyopharm Therapeutics Inc., Sera Prognostics Inc., and institutional financial interests (study grants)forAstraZeneca, Boehringer Ingelheim, Clovis Oncology, Pfizer, Tesaro-GSK, Ultimovacs; JL has reported advisory boards for AstraZeneca, Pfizer, GSK, Eisai, MSD/Merck, Artios Pharma, Regeneron, Amgen and Clovis Oncology, and grants for travelling from Clovis Oncology; CC has reported advisory boards for MSD, Takeda and GSK, conducting research for TherAguiX and Roche, and grants for travelling from Takeda; AF has reported advisory boards for GSK and Johnson \& Johnson SpA, and grants for travelling from Pharmmar and MSD Italia; CF has reported advisory boards for AstraZeneca, Clovis, Ethicon, Roche, MSD, GSK and Tesaro, and grants fro travelling from Sequana; AGM has reported speakers' bureau activities for AstraZeneca, Pharmamar, Roche and GSK, advisory boards for Amgen, AstraZeneca, Clovis Oncology, genmab, GSK, Immunogen, Merck Sharp \& Dohme, Novartis, Oncoinvent, Pfizer/Merck, Pharmamar, Roche and Sotio, and grants for travelling from AstraZeneca, Pharmamar Roche and Tesaro; DL has reported advisory boards for Roche, Amgen, MSD, GSK, Clovis, AstraZeneca, Immunogen, Genmab, Pharmamar and Merck, and grants for travelling from Pharmamar, GSK, Roche and AstraZeneca; CM has reported consulting/advisory boards for Roche, Novartis, Amgen, MSD, AstraZeneca, Pfizer, Pharmamar, Cerulean, Vertex and Tesaro, funded research from EU, FWF, AstraZeneca and Roche, and honoraria/expenses from Roche, Novartis, Amgen, MSD, Pharmamar, AstraZeneca and Tesaro; JS has reported advisory boards for Roche, Eisei, MSD, AstraZeneca, Clovis, GSK and Tesaro; AT has reported advisory boards for Genmab; PW has reported advisory boards for Amgen, AstraZeneca, MSD, Novartis, Pfizer, Pharmamar, Lilly, Roche Pharma GmbH, TEVA, Eisai, Clovis and Tesaro, and grants for travelling from Roche Pharma GmbH, AstraZeneca, MSD, Amgen and Pfizer; NC has reported consulting and advisory services, speaking or writing engagements, public presentations for Roche, AstraZeneca, MSD, Pharmamar, Tesaro, GSK, Clovis, Advaxis, Pfizer, Takeda, Immunogen, Biocad, Amgen, Novartis and Ellipses, institutional financial interests for Roche, Pharmamar and AstraZeneca, and non-financial interests for ESMO clinical Guidelines (subject editor for gynecological cancer); XMG, SM, TB, SL, PM, RN, DOD, DQ, MRR, AS, AW, FP and CC have reported no conflicts of interest. 


\section{REFERENCES}

1. World Health Organization. GLOBOCAN 2018: Estimated cancer incidence, mortality and prevalence worldwide in 2018.

2018; http://gco.iarc.fr/today/data/factsheets /cancers/24-Corpus-uteri-fact-sheet.pdf (29 July 2020, date last accessed).

2. Sant, M., et al. Survival of women with cancers of breast and genital organs in Europe 1999-2007: Results of the EUROCARE-5 study. Eur J Cancer 51, 2191-2205 (2015).

3. Colombo, N., et al. ESMO-ESGO-ESTRO Consensus Conference on Endometrial Cancer: Diagnosis, Treatment and Follow-up. Int J Gynecol Cancer 26, 2-30 (2016).

4. Colombo, N., et al. ESMO-ESGO-ESTRO consensus conference on endometrial cancer: Diagnosis, treatment and followup. Radiother Oncol 117, 559-581 (2015).

5. Colombo, N., et al. ESMO-ESGO-ESTRO Consensus Conference on Endometrial Cancer: diagnosis, treatment and followup. Ann Oncol 27, 16-41 (2016).

6. Colombo, N., et al. Endometrial cancer: ESMO Clinical Practice Guidelines for diagnosis, treatment and follow-up. Ann Oncol 24 Suppl 6, vi33-38 (2013).

7. Dykewicz, C.A. Summary of the Guidelines for Preventing Opportunistic Infections among Hematopoietic Stem Cell Transplant Recipients. Clin Infect Dis 33, 139-144 (2001).

8. Ryan, N.A.J., et al. The proportion of endometrial cancers associated with Lynch syndrome: a systematic review of the literature and meta-analysis. Genet Med (2019).

9. Cho, K.R., et al. International Society of Gynecological Pathologists (ISGyP) Endometrial Cancer Project: Guidelines
From the Special Techniques and Ancillary Studies Group. Int J Gynecol Pathol 38 Suppl 1, S114-S122 (2019).

10. Crosbie, E.J., et al. The Manchester International Consensus Group recommendations for the management of gynecological cancers in Lynch syndrome. Genet Med 21, 2390-2400 (2019).

11. Mills, A.M., et al. Lynch syndrome screening should be considered for all patients with newly diagnosed endometrial cancer. Am J Surg Pathol 38, 1501-1509 (2014).

12. Mojtahed, A., Schrijver, I., Ford, J.M., Longacre, T.A. \& Pai, R.K. A twoantibody mismatch repair protein immunohistochemistry screening approach for colorectal carcinomas, skin sebaceous tumors, and gynecologic tract carcinomas. Mod Pathol 24, 1004-1014 (2011).

13. Shia, J., et al. Immunohistochemistry as first-line screening for detecting colorectal cancer patients at risk for hereditary nonpolyposis colorectal cancer syndrome: a 2-antibody panel may be as predictive as a 4-antibody panel. Am J Surg Pathol 33, 1639-1645 (2009).

14. Moller, P., et al. Cancer incidence and survival in Lynch syndrome patients receiving colonoscopic and gynaecological surveillance: first report from the prospective Lynch syndrome database. Gut 66, 464-472 (2017).

15. Ryan, N.A.J., et al. Association of Mismatch Repair Mutation With Age at Cancer Onset in Lynch Syndrome: Implications for Stratified Surveillance Strategies. JAMA Oncol 3, 1702-1706 (2017).

16. Lachiewicz, M.P., et al. Prevalence of occult gynecologic malignancy at the 
time of risk reducing and nonprophylactic surgery in patients with Lynch syndrome. Gynecol Oncol 132, 434-437 (2014).

17. Kandoth, C., et al. Integrated genomic characterization of endometrial carcinoma. Nature 497, 67-73 (2013).

18. Piulats, J.M., et al. Molecular approaches for classifying endometrial carcinoma. Gynecol Oncol 145, 200-207 (2017).

19. Talhouk, A., et al. A clinically applicable molecular-based classification for endometrial cancers. Br J Cancer 113, 299-310 (2015).

20. Talhouk, A., et al. Confirmation of ProMisE: A simple, genomics-based clinical classifier for endometrial cancer. Cancer 123, 802-813 (2017).

21. Stelloo, E., et al. Improved Risk Assessment by Integrating Molecular and Clinicopathological Factors in Earlystage Endometrial Cancer-Combined Analysis of the PORTEC Cohorts. Clin Cancer Res 22, 4215-4224 (2016).

22. Leon-Castillo, A., et al. Molecular Classification of the PORTEC-3 Trial for High-Risk Endometrial Cancer: Impact on Prognosis and Benefit From Adjuvant Therapy. J Clin Oncol, JCO2000549 (2020).

23. Leon-Castillo, A., et al. Clinicopathological and molecular characterisation of 'multiple-classifier' endometrial carcinomas. J Pathol 250, 312-322 (2020).

24. Vermij, L., Smit, V., Nout, R. \& Bosse, T. Incorporation of molecular characteristics into endometrial cancer management. Histopathology 76, 52-63 (2020).

25. Church, D.N., et al. Prognostic significance of POLE proofreading mutations in endometrial cancer. J Natl Cancer Inst 107, 402 (2015).
26. Leon-Castillo, A., et al. Interpretation of somatic POLE mutations in endometrial carcinoma. J Pathol 250, 323-335 (2020).

27. McAlpine, J., Leon-Castillo, A. \& Bosse, T. The rise of a novel classification system for endometrial carcinoma; integration of molecular subclasses. $J$ Pathol 244, 538-549 (2018).

28. Kobel, M. \& Nelson, G.S. Letter in response to: McAlpine J, Leon-Castillo A, Bosse $\mathrm{T}$. The rise of a novel classification system for endometrial carcinoma; integration of molecular subclasses. J Pathol 2018; 244: 538-549. J Pathol 245, 249-250 (2018).

29. Kommoss, F.K., et al. L1CAM further stratifies endometrial carcinoma patients with no specific molecular risk profile. Br J Cancer 119, 480-486 (2018).

30. van der Putten, L.J., et al. L1CAM expression in endometrial carcinomas: an ENITEC collaboration study. $\mathrm{Br} J$ Cancer 115, 716-724 (2016).

31. Van Gool, I.C., et al. Prognostic significance of L1CAM expression and its association with mutant p53 expression in high-risk endometrial cancer. Mod Pathol 29, 174-181 (2016).

32. Bosse, T., et al. L1 cell adhesion molecule is a strong predictor for distant recurrence and overall survival in early stage endometrial cancer: pooled PORTEC trial results. Eur J Cancer 50, 2602-2610 (2014).

33. WHO Classification of Tumours, 5th Edition: Female Genital Organ Tumours, International Agency for Research on Cancer (IARC), Lyon (in press). (2020).

34. Ali, A., Black, D. \& Soslow, R.A. Difficulties in assessing the depth of myometrial invasion in endometrial carcinoma. Int J Gynecol Pathol 26, 115123 (2007). 
35. Luomaranta, A., Leminen, A. \& Loukovaara, M. Magnetic resonance imaging in the assessment of high-risk features of endometrial carcinoma: a meta-analysis. Int J Gynecol Cancer 25, 837-842 (2015).

36. Andreano, A., et al. MR diffusion imaging for preoperative staging of myometrial invasion in patients with endometrial cancer: a systematic review and metaanalysis. Eur Radiol 24, 1327-1338 (2014).

37. Das, S.K., et al. Usefulness of DWI in preoperative assessment of deep myometrial invasion in patients with endometrial carcinoma: a systematic review and meta-analysis. Cancer Imaging 14, 32 (2014).

38. Deng, L., et al. The Combination of Diffusion- and T2-Weighted Imaging in Predicting Deep Myometrial Invasion of Endometrial Cancer: A Systematic Review and Meta-Analysis. J Comput Assist Tomogr 39, 661-673 (2015).

39. Alcazar, J.L., et al. Transvaginal ultrasound versus magnetic resonance imaging for preoperative assessment of myometrial infiltration in patients with endometrial cancer: a systematic review and meta-analysis. J Gynecol Oncol 28, e86 (2017).

40. Tanaka, T., et al. Preoperative diffusionweighted magnetic resonance imaging and intraoperative frozen sections for predicting the tumor grade in endometrioid endometrial cancer. Oncotarget 9, 36575-36584 (2018).

41. Sanchez, M.F., et al. Diagnostic yield of magnetic resonance imaging and intraoperative frozen section in the determination of deep myometrial invasion in endometrial cancer. Radiologia 61, 315-323 (2019).

42. Fasmer, K.E., et al. Preoperative quantitative dynamic contrast-enhanced MRI and diffusion-weighted imaging predict aggressive disease in endometrial cancer. Acta Radiol 59, 1010-1017 (2018).

43. Taufiq, M., Masroor, I. \& Hussain, Z. Diagnostic Accuracy of Diffusion Weighted Magnetic Resonance Imaging in the Detection of Myometrial Invasion in Endometrial Carcinoma. J Coll Physicians Surg Pak 26, 13-17 (2016).

44. Christensen, J.W., et al. Assessment of myometrial invasion in endometrial cancer using three-dimensional ultrasound and magnetic resonance imaging. Acta Obstet Gynecol Scand 95, 55-64 (2016).

45. Arnaiz, J., Munoz, A.B., Verna, V., Gonzalez-Rodilla, I. \& Schneider, J. Magnetic Resonance Imaging for the Pre-Surgical Assessment of Endometrial Cancer: Results in a Routine Clinical Setting, Outside Dedicated Trials; a Cross-sectional Study. Anticancer Res 36, 1891-1894 (2016).

46. Shrivastava, S., et al. Magnetic resonance imaging in pre-operative staging of endometrial cancer. Indian J Cancer 53, 181-185 (2016).

47. Body, N., et al. Are preoperative histology and MRI useful for classification of endometrial cancer risk? BMC Cancer 16, 498 (2016).

48. Rodriguez-Trujillo, A., et al. Preoperative Assessment of Myometrial Invasion in Endometrial Cancer by 3D Ultrasound and Diffusion-Weighted Magnetic Resonance Imaging: A Comparative Study. Int J Gynecol Cancer 26, 1105-1110 (2016).

49. Horvath, K., Pete, I., Vereczkey, I., Dudnyikova, A. \& Godeny, M. Evaluation of the accuracy of preoperative MRI in measuring myometrial infiltration in endometrial carcinoma. Pathol Oncol Res 20, 327-333 (2014).

50. Nougaret, S., et al. Endometrial Cancer: Combined MR Volumetry and Diffusion- 
weighted Imaging for Assessment of Myometrial and Lymphovascular Invasion and Tumor Grade. Radiology 276, 797-808 (2015).

51. Ippolito, D., et al. Semiquantitative perfusion combined with diffusionweighted MR imaging in pre-operative evaluation of endometrial carcinoma: results in a group of 57 patients. Magn Reson Imaging 32, 464-472 (2014).

52. Tanaka, T., et al. Preoperative MRI and intraoperative frozen section diagnosis of myometrial invasion in patients with endometrial cancer. Int J Gynecol Cancer 25, 879-883 (2015).

53. Bonatti, M., et al. MRI for local staging of endometrial carcinoma: Is endovenous contrast medium administration still needed? Eur J Radiol 84, 208-214 (2015).

54. Karatasli, V., Cakir, I., Sahin, H., Ayaz, D. \& Sanci, M. Can preoperative magnetic resonance imaging replace intraoperative frozen sectioning in the evaluation of myometrial invasion for early-stage endometrial carcinoma? Ginekol Pol 90, 128-133 (2019).

55. Fujii, S., et al. Subendometrial enhancement and peritumoral enhancement for assessing endometrial cancer on dynamic contrast enhanced MR imaging. Eur J Radiol 84, 581-589 (2015).

56. Yang, T., et al. Magnetic Resonance Imaging (MRI) and Three-Dimensional Transvaginal Ultrasonography Scanning for Preoperative Assessment of High Risk in Women with Endometrial Cancer. Med Sci Monit 25, 2024-2031 (2019).

57. Ahmed, M., et al. Can MRI help assess aggressiveness of endometrial cancer? Clin Radiol 73, 833 e811-833 e818 (2018).

58. Sahin, H., et al. Preoperative Magnetic Resonance Volumetry in Predicting
Myometrial Invasion, Lymphovascular Space Invasion, and Tumor Grade: Is It Valuable in International Federation of Gynecology and Obstetrics Stage I Endometrial Cancer? Int J Gynecol Cancer 28, 666-674 (2018).

59. Yan, B., Zhao, T., Liang, X., Niu, C. \& Ding, C. Can the apparent diffusion coefficient differentiate the grade of endometrioid adenocarcinoma and the histological subtype of endometrial cancer? Acta Radiol 59, 363-370 (2018).

60. Zhang, L., et al. Use of diffusion tensor imaging in assessing superficial myometrial invasion by endometrial carcinoma: a preliminary study. Acta Radiol 56, 1273-1280 (2015).

61. Bonatti, M., et al. Prediction of histological grade of endometrial cancer by means of MRI. Eur J Radiol 103, 4450 (2018).

62. Tsikouras, P., et al. Preoperative assessment in endometrial cancer. Is triage for lymphadenectomy possible? $\mathrm{J}$ BUON 22, 34-43 (2017).

63. Zamani, N., Modares Gilani, M., Zamani, F. \& Zamani, M.H. Utility of Pelvic MRI and Tumor Markers HE4 and CA125 to Predict Depth of Myometrial Invasion and Cervical Involvement in Endometrial Cancer. J Family Reprod Health 9, 177-183 (2015).

64. Bourgioti, C., et al. Predictive ability of maximal tumor diameter on MRI for high-risk endometrial cancer. Abdom Radiol (NY) 41, 2484-2495 (2016).

65. Ytre-Hauge, S., et al. Preoperative tumor texture analysis on MRI predicts highrisk disease and reduced survival in endometrial cancer. J Magn Reson Imaging 48, 1637-1647 (2018).

66. Thieme, S.F., et al. Preoperative Evaluation of Myometrial Invasion in Endometrial Carcinoma: Prospective Intra-individual Comparison of Magnetic Resonance Volumetry, 
Diffusion-weighted and Dynamic Contrast-enhanced Magnetic Resonance Imaging. Anticancer Res 38, 4813-4817 (2018).

67. Deng, L., et al. Combined subjective and quantitative analysis of magnetic resonance images could improve the diagnostic performance of deep myometrial invasion in endometrial cancer. Clin Imaging 43, 69-73 (2017).

68. Gallego, J.C., Porta, A., Pardo, M.C. \& Fernandez, C. Evaluation of myometrial invasion in endometrial cancer: comparison of diffusion-weighted magnetic resonance and intraoperative frozen sections. Abdom Imaging 39, 1021-1026 (2014).

69. Brocker, K.A., et al. Comparison of the determination of the local tumor extent of primary endometrial cancer using clinical examination and 3 Tesla magnetic resonance imaging compared to histopathology. Arch Gynecol Obstet 299, 1391-1398 (2019).

70. Goel, G., Rajanbabu, A., Sandhya, C.J. \& Nair, I.R. A Prospective Observational Study Evaluating the Accuracy of MRI in Predicting the Extent of Disease in Endometrial Cancer. Indian J Surg Oncol 10, 220-224 (2019).

71. Cignini, P., et al. Preoperative work-up for definition of lymph node risk involvement in early stage endometrial cancer: 5-year follow-up. Updates Surg 69, 75-82 (2017).

72. Soneji, N.D., Bharwani, N., Ferri, A., Stewart, V. \& Rockall, A. Pre-operative MRI staging of endometrial cancer in a multicentre cancer network: can we match single centre study results? Eur Radiol 28, 4725-4734 (2018).

73. Green, R.W., et al. Endometrial cancer off-line staging using two-dimensional transvaginal ultrasound and threedimensional volume contrast imaging: Intermethod agreement, interrater reliability and diagnostic accuracy. Gynecol Oncol 150, 438-445 (2018).

74. Takeuchi, M., Matsuzaki, K. \& Harada, M. Evaluating Myometrial Invasion in Endometrial Cancer: Comparison of Reduced Field-of-view Diffusionweighted Imaging and Dynamic Contrast-enhanced MR Imaging. Magn Reson Med Sci 17, 28-34 (2018).

75. Ota, $\mathrm{T}$., et al. Preoperative staging of endometrial cancer using reduced fieldof-view diffusion-weighted imaging: a preliminary study. Eur Radiol 27, 52255235 (2017).

76. Koplay, M., et al. Diagnostic efficacy of diffusion-weighted MRI for preoperative assessment of myometrial and cervical invasion and pelvic lymph node metastasis in endometrial carcinoma. $J$ Med Imaging Radiat Oncol 58, 538-546; quiz 648 (2014).

77. Woo, S., Kim, S.Y., Cho, J.Y. \& Kim, S.H. Assessment of deep myometrial invasion of endometrial cancer on MRI: added value of second-opinion interpretations by radiologists subspecialized in gynaecologic oncology. Eur Radiol 27, 1877-1882 (2017).

78. Alves, I. \& Cunha, T.M. Clinical importance of second-opinion interpretations by radiologists specializing in gynecologic oncology at a tertiary cancer center: magnetic resonance imaging for endometrial cancer staging. Radiol Bras 51, 26-31 (2018).

79. Masroor, I., Rashid, S., Afzal, S., Sufian, S.N. \& Azeemuddin, M. Diagnostic Accuracy of Pelvic MRI for Determination of the Cervical Involvement in Endometrial Cancer. $J$ Coll Physicians Surg Pak 28, 262-265 (2018).

80. Teng, F., et al. Contrast-enhanced MRI in preoperative assessment of myometrial 
and cervical invasion, and lymph node metastasis: diagnostic value and error analysis in endometrial carcinoma. Acta Obstet Gynecol Scand 94, 266-273 (2015).

81. Bhosale, P., et al. Feasibility of a reduced field-of-view diffusion-weighted (rFOV) sequence in assessment of myometrial invasion in patients with clinical FIGO stage I endometrial cancer.J Magn Reson Imaging 43, 316-324 (2016).

82. Lin, G., et al. Endometrial cancer with cervical stromal invasion: diagnostic accuracy of diffusion-weighted and dynamic contrast enhanced MR imaging at 3T. Eur Radiol 27, 1867-1876 (2017).

83. Alcazar, J.L., et al. Transvaginal/transrectal ultrasound for assessing myometrial invasion in endometrial cancer: a comparison of six different approaches. J Gynecol Oncol 26, 201-207 (2015).

84. Eriksson, L.S., et al. Transvaginal ultrasound assessment of myometrial and cervical stromal invasion in women with endometrial cancer: interobserver reproducibility among ultrasound experts and gynecologists. Ultrasound Obstet Gynecol 45, 476-482 (2015).

85. Vieillefosse, S., et al. Assessment of different pre and intra-operative strategies to predict the actual ESMO risk group and to establish the appropriate indication of lymphadenectomy in endometrial cancer. J Gynecol Obstet Hum Reprod 47, 517-523 (2018).

86. Jantarasaengaram, S., Praditphol, N., Tansathit, T., Vipupinyo, C. \& Vairojanavong, K. Three-dimensional ultrasound with volume contrast imaging for preoperative assessment of myometrial invasion and cervical involvement in women with endometrial cancer. Ultrasound Obstet Gynecol 43, 569-574 (2014).
87. Pineda, L., et al. Agreement between preoperative transvaginal ultrasound and intraoperative macroscopic examination for assessing myometrial infiltration in low-risk endometrioid carcinoma. Ultrasound Obstet Gynecol 47, 369-373 (2016).

88. Fruhauf, F., et al. The Diagnostic Accuracy of Ultrasound in Assessment of Myometrial Invasion in Endometrial Cancer: Subjective Assessment versus Objective Techniques. Biomed Res Int 2017, 1318203 (2017).

89. Bollineni, V.R., Ytre-Hauge, S., BollineniBalabay, O., Salvesen, H.B. \& Haldorsen, I.S. High Diagnostic Value of 18F-FDG $\mathrm{PET} / \mathrm{CT}$ in Endometrial Cancer: Systematic Review and Meta-Analysis of the Literature. J Nucl Med 57, 879-885 (2016).

90. Legros, M., et al. Para-aortic Lymph Node Invasion in High-risk Endometrial Cancer: Performance of (18)FDG PETCT. Anticancer Res 39, 619-625 (2019).

91. Kim, H.J., Cho, A., Yun, M., Kim, Y.T. \& Kang, W.J. Comparison of FDG PET/CT and MRI in lymph node staging of endometrial cancer. Ann Nucl Med 30, 104-113 (2016).

92. Chung, H.H., et al. Preoperative PET/CT standardized FDG uptake values of pelvic lymph nodes as a significant prognostic factor in patients with endometrial cancer. Eur J Nucl Med Mol Imaging 41, 1793-1799 (2014).

93. Konuralp Atakul, B., et al. Preoperative 18F-Fluorodeoxyglucose Positron Emission Tomography/CT in Prediction of Uterine Risk Factors and Lymph Node Metastasis: An Analysis of 111 Endometrioid Endometrial Cancer Patients. Gynecol Obstet Invest 82, 340348 (2017).

94. Kulkarni, R., Bhat, R.A., Dhakharia, V., Kallur, K. \& Gangoli, A. Role of Positron Emission Tomography/Computed 
Tomography in Preoperative Assessment of Carcinoma Endometrium-a Retrospective Analysis. Indian J Surg Oncol 10, 225-231 (2019).

95. Tanaka, T., Terai, Y., Yamamoto, K., Yamada, T. \& Ohmichi, M. The diagnostic accuracy of fluorodeoxyglucosepositron emission tomography/computed tomography and sentinel node biopsy in the prediction of pelvic lymph node metastasis in patients with endometrial cancer: A retrospective observational study. Medicine (Baltimore) 97, e12522 (2018).

96. Bese, T., et al. The Combination of Preoperative Fluorodeoxyglucose Positron Emission Tomography/Computed Tomography and Sentinel Lymph Node Mapping in the Surgical Management of Endometrioid Endometrial Cancer. Int J Gynecol Cancer 26, 1228-1238 (2016).

97. Park, J.Y., et al. The Value of Preoperative Positron Emission Tomography/Computed Tomography in Node-Negative Endometrial Cancer on Magnetic Resonance Imaging. Ann Surg Oncol 24, 2303-2310 (2017).

98. Gulseren, V., et al. Is the measurement of the size of uterine lesions with positron emission tomography consistent in preand postmenopausal periods in endometrioid-type endometrial cancer? Turk J Obstet Gynecol 15, 60-64 (2018).

99. Gholkar, N.S., et al. The Accuracy of Integrated [(18)F] FluorodeoxyglucosePositron Emission Tomography/Computed Tomography in Detection of Pelvic and Para-aortic Nodal Metastasis in Patients with High Risk Endometrial Cancer. World J Nucl Med 13, 170-177 (2014).

100. Mayoral, M., et al. (18)F-FDG PET/CT and sentinel lymph node biopsy in the staging of patients with cervical and endometrial cancer. Role of dual-time- point imaging. Rev Esp Med Nucl Imagen Mol 36, 20-26 (2017).

101. Ghooshkhanei, H., Treglia, G., Sabouri, G., Davoodi, R. \& Sadeghi, R. Risk stratification and prognosis determination using (18)F-FDG PET imaging in endometrial cancer patients: a systematic review and meta-analysis. Gynecol Oncol 132, 669-676 (2014).

102. Dai, S., et al. Impact and cost of preoperative computed tomography imaging on the management of patients diagnosed with high-grade endometrial cancer. Int J Gynaecol Obstet 145, 219224 (2019).

103. Bogani, G., et al. Clinical Utility of Preoperative Computed Tomography in Patients With Endometrial Cancer. Int J Gynecol Cancer 27, 1685-1693 (2017).

104. Janda, M., et al. Effect of Total Laparoscopic Hysterectomy vs Total Abdominal Hysterectomy on DiseaseFree Survival Among Women With Stage I Endometrial Cancer: A Randomized Clinical Trial. JAMA 317, 1224-1233 (2017).

105. Walker, J.L., et al. Recurrence and survival after random assignment to laparoscopy versus laparotomy for comprehensive surgical staging of uterine cancer: Gynecologic Oncology Group LAP2 Study. J Clin Oncol 30, 695700 (2012).

106. Togami, S., et al. Learning curve and surgical outcomes for laparoscopic surgery, including pelvic lymphadenectomy, for early stage endometrial cancer. Jpn J Clin Oncol 49, 521-524 (2019).

107. Deura, I., et al. Comparison of laparoscopic surgery and conventional laparotomy for surgical staging of patients with presumed low-risk endometrial cancer: The current state of Japan. Taiwan J Obstet Gynecol 58, 99104 (2019). 
108. Ghazali, W., Jamil, S.A. \& Sharin, I.A. Laparoscopic versus Laparotomy: Staging Surgery for Endometrial Cancer - Malaysia's Early Experience. Gynecol Minim Invasive Ther 8, 25-29 (2019).

109. Vardar, M.A., et al. Laparoscopic surgery for low, intermediate and high-risk endometrial cancer. J Gynecol Oncol 30, e24 (2019).

110. Pookunju, A.P. \& Ayyappan, S. Technique of Laparoscopic Hysterectomy and Pelvic Lymphadenectomy for Endometrial Cancer. Indian J Surg Oncol 9, 290-293 (2018).

111. Wollinga, T., et al. Implementation of laparoscopic hysterectomy for endometrial cancer over the past decade. Gynecol Surg 15, 7 (2018).

112. Van den Bosch, A. \& Mertens, H. Implementation of laparoscopic surgery for endometrial cancer: work in progress. Facts Views Vis Obgyn 8, 23-30 (2016).

113. Chu, L.H., Chang, W.C. \& Sheu, B.C. Comparison of the laparoscopic versus conventional open method for surgical staging of endometrial carcinoma. Taiwan J Obstet Gynecol 55, 188-192 (2016).

114. Favero, G., et al. Oncologic Safety of Laparoscopy in the Surgical Treatment of Type II Endometrial Cancer. Int J Gynecol Cancer 26, 1673-1678 (2016).

115. Bennich, G., Rudnicki, M. \& Lassen, P.D. Laparoscopic surgery for early endometrial cancer. Acta Obstet Gynecol Scand 95, 894-900 (2016).

116. Lee, C.L., et al. Long-term survival outcomes of laparoscopic staging surgery in treating endometrial cancer: 20 years of follow-up. Taiwan J Obstet Gynecol 55, 545-551 (2016).

117. Berretta, R., et al. Quality of life in patients affected by endometrial cancer: comparison among laparotomy, laparoscopy and vaginal approach. Pathol Oncol Res 21, 811-816 (2015).

118. Yin, X., Shi, M., Xu, J., Guo, Q. \& Wu, H. Perioperative and long-term outcomes of laparoscopy and laparotomy for endometrial carcinoma. Int J Clin Exp Med 8, 19093-19099 (2015).

119. Kroft, J., et al. Trends over time in the use of laparoscopic hysterectomy for the treatment of endometrial cancer. Gynecol Oncol 138, 536-541 (2015).

120. Pawlowicz, P.S. \& Ajdacka, U. The role of laparoscopy in the surgical treatment of endometrial cancer. Wideochir Inne Tech Maloinwazyjne 10, 44-48 (2015).

121. Gao, H. \& Zhang, Z. Laparoscopy Versus Laparotomy in the Treatment of HighRisk Endometrial Cancer: A Propensity Score Matching Analysis. Medicine (Baltimore) 94, e1245 (2015).

122. Senol, T., Polat, M., Sanverdi, I., Ozkaya, E. \& Karateke, A. Laparoscopic staging of endometrial cancer: Does it have any impact on survival? Turk J Obstet Gynecol 12, 139-143 (2015).

123. Palomba, S., et al. Conversion in endometrial cancer patients scheduled for laparoscopic staging: a large multicenter analysis: conversions and endometrial cancer. Surg Endosc 28, 3200-3209 (2014).

124. Lee, C.L., Huang, K.G., Wu, P.J., Lee, P.S. \& Yen, C.F. Long-term survival outcome of laparoscopic staging surgery for endometrial cancer in Taiwanese experience. Taiwan J Obstet Gynecol 53, 57-61 (2014).

125. Terai, Y., et al. Total laparoscopic modified radical hysterectomy with lymphadenectomy for endometrial cancer compared with laparotomy. J Obstet Gynaecol Res 40, 570-575 (2014).

126. Koskas, M., et al. Long-term oncological safety of minimally invasive surgery in 
high-risk endometrial cancer. Eur J Cancer 65, 185-191 (2016).

127. Uccella, S., et al. Laparoscopic vs. open treatment of endometrial cancer in the elderly and very elderly: An agestratified multicenter study on 1606 women. Gynecol Oncol 141, 211-217 (2016).

128. Bogani, G., et al. Perioperative and longterm outcomes of laparoscopic, open abdominal, and vaginal surgery for endometrial cancer in patients aged 80 years or older. Int J Gynecol Cancer 24, 894-900 (2014).

129. Baek, M.H., et al. Feasibility and safety of laparoscopic surgery for obese Korean women with endometrial cancer: longterm results at a single institution. $J$ Korean Med Sci 29, 1536-1543 (2014).

130. Bogani, G., et al. Laparoscopic staging in women older than 75 years with earlystage endometrial cancer: comparison with open surgical operation. Menopause 21, 945-951 (2014).

131. Freeman, A.H., et al. Venous thromboembolism following minimally invasive surgery among women with endometrial cancer. Gynecol Oncol 142, 267-272 (2016).

132. Raventos-Tato, R.M., et al. Surgical approaches in women with endometrial cancer with a body mass index greater than $35 \mathrm{~kg} / \mathrm{m}(2)$. J Obstet Gynaecol Res 45, 195-202 (2019).

133. Bishop, E.A., et al. Surgical outcomes among elderly women with endometrial cancer treated by laparoscopic hysterectomy: a NRG/Gynecologic Oncology Group study. Am J Obstet Gynecol 218, 109 e101-109 e111 (2018).

134. Casarin, J., et al. Adoption of Minimally Invasive Surgery and Decrease in Surgical Morbidity for Endometrial Cancer Treatment in the United States. Obstet Gynecol 131, 304-311 (2018).
135. Ee, W.W., Nellore, V., McMullen, W. \& Ragupathy, K. Laparoscopic hysterectomy for endometrial cancer: impact of age on clinical outcomes. $J$ Obstet Gynaecol 38, 734 (2018).

136. Singh, S., Swarer, K. \& Resnick, K. Longer operative time is associated with increased post-operative complications in patients undergoing minimallyinvasive surgery for endometrial cancer. Gynecol Oncol 147, 554-557 (2017).

137. Bregar, A.J., et al. Minimally Invasive Staging Surgery in Women with EarlyStage Endometrial Cancer: Analysis of the National Cancer Data Base. Ann Surg Oncol 24, 1677-1687 (2017).

138. Monterossi, G., et al. Minimally Invasive Approach in Type II Endometrial Cancer: Is It Wise and Safe? J Minim Invasive Gynecol 24, 438-445 (2017).

139. Barber, E.L., Gehrig, P.A. \& ClarkePearson, D.L. Venous Thromboembolism in Minimally Invasive Compared With Open Hysterectomy for Endometrial Cancer. Obstet Gynecol 128, 121-126 (2016).

140. Pulman, K.J., et al. Comparison of three surgical approaches for staging lymphadenectomy in high-risk endometrial cancer. Int $J$ Gynaecol Obstet 136, 315-319 (2017).

141. Marcos-Sanmartin, J., et al. Does the Type of Surgical Approach and the Use of Uterine Manipulators Influence the Disease-Free Survival and Recurrence Rates in Early-Stage Endometrial Cancer? Int J Gynecol Cancer 26, 17221726 (2016).

142. Tanaka, T., et al. Comparison Between Laparoscopy and Laparotomy in Systematic Para-Aortic Lymphadenectomy for Patients with Endometrial Cancer: A Retrospective Multicenter Study. J Gynecol Surg 33, 105-110 (2017). 
143. Galaal, K., Donkers, H., Bryant, A. \& Lopes, A.D. Laparoscopy versus laparotomy for the management of early stage endometrial cancer. Cochrane Database Syst Rev 10, CD006655 (2018).

144. Asher, R., Obermair, A., Janda, M. \& Gebski, V. Disease-Free and Survival Outcomes for Total Laparoscopic Hysterectomy Compared With Total Abdominal Hysterectomy in Early-Stage Endometrial Carcinoma: A Metaanalysis. Int J Gynecol Cancer 28, 529538 (2018).

145. Ansar, P.P., Ayyappan, S. \& Mahajan, V. Prospective Nonrandomized Comparative Study of Laparoscopic Versus Open Surgical Staging for Endometrial Cancer in India. Indian J Surg Oncol 9, 133-140 (2018).

146. Jorgensen, S.L., et al. Nationwide Introduction of Minimally Invasive Robotic Surgery for Early-Stage Endometrial Cancer and Its Association With Severe Complications. JAMA Surg (2019).

147. Kyrgiou, M., Swart, A.M., Qian, W. \& Warwick, J. A Comparison of Outcomes Following Laparoscopic and Open Hysterectomy With or Without Lymphadenectomy for Presumed EarlyStage Endometrial Cancer: Results From the Medical Research Council ASTEC Trial. Int J Gynecol Cancer 25, 14241436 (2015).

148. Park, D.A., Lee, D.H., Kim, S.W. \& Lee, S.H. Comparative safety and effectiveness of robot-assisted laparoscopic hysterectomy versus conventional laparoscopy and laparotomy for endometrial cancer: A systematic review and meta-analysis. Eur J Surg Oncol 42, 1303-1314 (2016).

149. Ran, L., Jin, J., Xu, Y., Bu, Y. \& Song, F. Comparison of robotic surgery with laparoscopy and laparotomy for treatment of endometrial cancer: a meta-analysis. PLoS One 9, e108361 (2014).

150. Nevis, I.F., et al. Robot-assisted hysterectomy for endometrial and cervical cancers: a systematic review. J Robot Surg 11, 1-16 (2017).

151. Lundin, E.S., Wodlin, N.B., Nilsson, L. \& Kjolhede, P. A prospective randomized assessment of quality of life between open and robotic hysterectomy in early endometrial cancer. Int J Gynecol Cancer (2019).

152. Herling, S.F., Moller, A.M., Palle, C., Grynnerup, A. \& Thomsen, T. Roboticassisted laparoscopic hysterectomy for women with endometrial cancer. Dan Med J 64(2017).

153. Uccella, S., et al. Impact of Obesity on Surgical Treatment for Endometrial Cancer: A Multicenter Study Comparing Laparoscopy vs Open Surgery, with Propensity-Matched Analysis. J Minim Invasive Gynecol 23, 53-61 (2016).

154. Corrado, G., et al. Robotic single site staging in endometrial cancer: A multiinstitution study. Eur J Surg Oncol 42, 1506-1511 (2016).

155. Backes, F.J., et al. Perioperative Outcomes for Laparotomy Compared to Robotic Surgical Staging of Endometrial Cancer in the Elderly: A Retrospective Cohort. Int J Gynecol Cancer 26, 17171721 (2016).

156. Guy, M.S., Sheeder, J., Behbakht, K., Wright, J.D. \& Guntupalli, S.R. Comparative outcomes in older and younger women undergoing laparotomy or robotic surgical staging for endometrial cancer. Am J Obstet Gynecol 214, 350 e351-350 e310 (2016).

157. Herling, S.F., Havemann, M.C., Palle, C., Moller, A.M. \& Thomsen, T. Roboticassisted laparoscopic hysterectomy seems safe in women with early-stage endometrial cancer. Dan Med J 62, A5109 (2015). 
158. Beck, T.L., Schiff, M.A., Goff, B.A. \& Urban, R.R. Robotic, Laparoscopic, or Open Hysterectomy: Surgical Outcomes by Approach in Endometrial Cancer. J Minim Invasive Gynecol 25, 986-993 (2018).

159. Doo, D.W., et al. Comparative Surgical Outcomes for Endometrial Cancer Patients 65 Years Old or Older Staged With Robotics or Laparotomy. Ann Surg Oncol 22, 3687-3694 (2015).

160. Park, H.K., Helenowski, I.B., Berry, E., Lurain, J.R. \& Neubauer, N.L. A Comparison of Survival and Recurrence Outcomes in Patients With Endometrial Cancer Undergoing Robotic Versus Open Surgery. J Minim Invasive Gynecol 22, 961-967 (2015).

161. Feuer, G.A., et al. Robotic surgery for staging of serous papillary and clear cell carcinoma of the endometrium. Int $J$ Med Robot 10, 306-313 (2014).

162. Pant, A., Schink, J. \& Lurain, J. Robotic surgery compared with laparotomy for high-grade endometrial cancer. J Robot Surg 8, 163-167 (2014).

163. Safdieh, J., et al. A Comparison of Outcomes Between Open Hysterectomy and Robotic-Assisted Hysterectomy for Endometrial Cancer Using the National Cancer Database. Int J Gynecol Cancer 27, 1508-1516 (2017).

164. Wright, J.D., et al. Comparative Effectiveness of Minimally Invasive Hysterectomy for Endometrial Cancer. J Clin Oncol 34, 1087-1096 (2016).

165. Barraez, D., Godoy, H., McElrath, T., Kredentser, D. \& Timmins, P. Low incidence of port-site metastasis after robotic assisted surgery for endometrial cancer staging: descriptive analysis. $J$ Robot Surg 9, 91-95 (2015).

166. Yoon, A., et al. Robotic single-port hysterectomy, adnexectomy, and lymphadenectomy in endometrial cancer. J Minim Invasive Gynecol 22, 322 (2015).

167. Geppert, B. \& Persson, J. Robotic infrarenal paraaortic and pelvic nodal staging for endometrial cancer: feasibility and lymphatic complications. Acta Obstet Gynecol Scand 94, 10741081 (2015).

168. Damiani, G.R., et al. Robotic approach using simple and radical hysterectomy for endometrial cancer with long-term follow-up evaluation. Int J Med Robot 12, 109-113 (2016).

169. Bige, O., Demir, A., Saatli, B., Koyuncuoglu, M. \& Saygili, U. Laparoscopy versus laparotomy for the management of endometrial carcinoma in morbidly obese patients: a prospective study. J Turk Ger Gynecol Assoc 16, 164-169 (2015).

170. Salehi, S., Avall-Lundqvist, E., Legerstam, B., Carlson, J.W. \& Falconer, H. Robotassisted laparoscopy versus laparotomy for infrarenal paraaortic lymphadenectomy in women with highrisk endometrial cancer: A randomised controlled trial. Eur J Cancer 79, 81-89 (2017).

171. Salehi, S., et al. Long-term quality of life after comprehensive surgical staging of high-risk endometrial cancer - results from the RASHEC trial. Acta Oncol 57, 1671-1676 (2018).

172. Signorelli, M., et al. Modified radical hysterectomy versus extrafascial hysterectomy in the treatment of stage I endometrial cancer: results from the ILIADE randomized study. Ann Surg Oncol 16, 3431-3441 (2009).

173. Kaban, A., et al. Is Omentectomy Necessary for Non-Endometrioid Endometrial Cancer. Gynecol Obstet Invest 83, 482-486 (2018).

174. Joo, W.D., et al. Microscopic Omental Metastasis in Clinical Stage I 
Endometrial Cancer: A Meta-analysis. Ann Surg Oncol 22, 3695-3700 (2015).

175. Ross, M.S., Elishaev, E., Berger, J.L., Kelley, J.L. \& Taylor, S.E. Prognostic Significance of omental Disease and the Role of Omental Sampling in Patients With Uterine Carcinosarcoma. Int $J$ Gynecol Cancer 28, 254-259 (2018).

176. Lee, B., Suh, D.H., Kim, K., No, J.H. \& Kim, Y.B. Influence of positive peritoneal cytology on prognostic factors and survival in early-stage endometrial cancer: a systematic review and metaanalysis. Jpn J Clin Oncol 46, 711-717 (2016).

177. Matsuo, K., et al. Significance of abnormal peritoneal cytology on survival of women with stage I-II endometrioid endometrial cancer. Gynecol Oncol 149, 301-309 (2018).

178. Seagle, B.L., Alexander, A.L., Lantsman, T. \& Shahabi, S. Prognosis and treatment of positive peritoneal cytology in early endometrial cancer: matched cohort analyses from the National Cancer Database. Am J Obstet Gynecol 218, 329 e321-329 e315 (2018).

179. Bogani, G., Murgia, F., Ditto, A. \& Raspagliesi, F. Sentinel node mapping vs. lymphadenectomy in endometrial cancer: A systematic review and metaanalysis. Gynecol Oncol 153, 676-683 (2019).

180. Leitao, M.M., Jr. Sentinel Lymph Node Mapping in Patients with Endometrial Carcinoma: Less Can Be More. Curr Obstet Gynecol Rep 5, 279-285 (2016).

181. Rossi, E.C., et al. A comparison of sentinel lymph node biopsy to lymphadenectomy for endometrial cancer staging (FIRES trial): a multicentre, prospective, cohort study. Lancet Oncol 18, 384-392 (2017).

182. Persson, J., et al. Pelvic Sentinel lymph node detection in High-Risk Endometrial Cancer (SHREC-trial)-the final step towards a paradigm shift in surgical staging. Eur J Cancer 116, 77-85 (2019).

183. Darai, E., et al. Sentinel node biopsy for the management of early stage endometrial cancer: long-term results of the SENTI-ENDO study. Gynecol Oncol 136, 54-59 (2015).

184. Renz, M., et al. Immediate intraoperative sentinel lymph node analysis by frozen section is predictive of lymph node metastasis in endometrial cancer. $J$ Robot Surg (2019).

185. How, J.A., et al. Sentinel lymph node mapping in endometrial cancer: a systematic review and meta-analysis. Minerva Ginecol 70, 194-214 (2018).

186. Lin, H., Ding, Z., Kota, V.G., Zhang, X. \& Zhou, J. Sentinel lymph node mapping in endometrial cancer: a systematic review and meta-analysis. Oncotarget 8, 4660146610 (2017).

187. Staley, A., Sullivan, S.A. \& Rossi, E.C. Sentinel Lymph Node Technique in Endometrial Cancer. Obstet Gynecol Surv 72, 289-295 (2017).

188. Tschernichovsky, R., Diver, E.J., Schorge, J.O. \& Goodman, A. The Role of Lymphadenectomy Versus Sentinel Lymph Node Biopsy in Early-stage Endometrial Cancer: A Review of the Literature. Am J Clin Oncol 39, 516-521 (2016).

189. Bodurtha Smith, A.J., Fader, A.N. \& Tanner, E.J. Sentinel lymph node assessment in endometrial cancer: a systematic review and meta-analysis. Am J Obstet Gynecol 216, 459-476 e410 (2017).

190. Wang, L. \& Liu, F. Meta-analysis of laparoscopy sentinel lymph node mapping in endometrial cancer. Arch Gynecol Obstet 298, 505-510 (2018).

191. Baiocchi, G., et al. The Impact of Sentinel Node-Mapping in Staging High-Risk 
Endometrial Cancer. Ann Surg Oncol 24, 3981-3987 (2017).

192. Tanner, E., et al. Use of a novel sentinel lymph node mapping algorithm reduces the need for pelvic lymphadenectomy in low-grade endometrial cancer. Gynecol Oncol 147, 535-540 (2017).

193. Martinelli, F., et al. Sentinel node mapping in endometrial cancer following Hysteroscopic injection of tracers: A single center evaluation over 200 cases. Gynecol Oncol 146, 525-530 (2017).

194. Buda, A., et al. The impact on survival of two different staging strategies in apparent early stage endometrial cancer comparing sentinel lymph nodes mapping algorithm and selective lymphadenectomy: An Italian retrospective analysis of two reference centers. Gynecol Oncol 147, 528-534 (2017).

195. Yamagami, W., et al. A Comparison of Dye Versus Fluorescence Methods for Sentinel Lymph Node Mapping in Endometrial Cancer. Int J Gynecol Cancer 27, 1517-1524 (2017).

196. Touhami, O., Gregoire, J., Renaud, M.C., Sebastianelli, A. \& Plante, M. Performance of sentinel lymph node (SLN) mapping in high-risk endometrial cancer. Gynecol Oncol 147, 549-553 (2017).

197. Papadia, A., et al. The impact of different doses of indocyanine green on the sentinel lymph-node mapping in early stage endometrial cancer. J Cancer Res Clin Oncol 144, 2187-2191 (2018).

198. Eoh, K.J., et al. Two-step sentinel lymph node mapping strategy in endometrial cancer staging using fluorescent imaging: A novel sentinel lymph node tracer injection procedure. Surg Oncol 27, 514-519 (2018).

199. Ducie, J.A., et al. Comparison of a sentinel lymph node mapping algorithm and comprehensive lymphadenectomy in the detection of stage IIIC endometrial carcinoma at higher risk for nodal disease. Gynecol Oncol 147, 541548 (2017).

200. Tanner, E.J., et al. The Utility of Sentinel Lymph Node Mapping in High-Grade Endometrial Cancer. Int J Gynecol Cancer 27, 1416-1421 (2017).

201. How, J., et al. Impact of sentinel lymph node mapping on recurrence patterns in endometrial cancer. Gynecol Oncol 144, 503-509 (2017).

202. Papadia, A., et al. Sentinel lymph node mapping in patients with stage I endometrial carcinoma: a focus on bilateral mapping identification by comparing radiotracer $\mathrm{Tc} 99(\mathrm{~m})$ with blue dye versus indocyanine green fluorescent dye. J Cancer Res Clin Oncol 143, 475-480 (2017).

203. Tanaka, T., et al. The detection of sentinel lymph nodes in laparoscopic surgery can eliminate systemic lymphadenectomy for patients with early stage endometrial cancer. Int J Clin Oncol 23, 305-313 (2018).

204. Buda, A., et al. Lymph node evaluation in high-risk early stage endometrial cancer: A multi-institutional retrospective analysis comparing the sentinel lymph node (SLN) algorithm and SLN with selective lymphadenectomy. Gynecol Oncol 150, 261-266 (2018).

205. Buda, A., et al. Sentinel Lymph Node Mapping With Near-Infrared Fluorescent Imaging Using Indocyanine Green: A New Tool for Laparoscopic Platform in Patients With Endometrial and Cervical Cancer. J Minim Invasive Gynecol 23, 265-269 (2016).

206. Buda, A., et al. Optimizing Strategies for Sentinel Lymph Node Mapping in EarlyStage Cervical and Endometrial Cancer: Comparison of Real-Time Fluorescence 
With Indocyanine Green and Methylene Blue. Int J Gynecol Cancer 25, 1513-1518 (2015).

207. Signorelli, M., et al. Staging of High-Risk Endometrial Cancer With PET/CT and Sentinel Lymph Node Mapping. Clin Nucl Med 40, 780-785 (2015).

208. Rajanbabu, A., Venkatesan, R., Chandramouli, S. \& Nitu, P.V. Sentinel node detection in endometrial cancer using indocyanine green and fluorescence imaging-a case report. Ecancermedicalscience 9, 549 (2015).

209. Surynt, E., Reinholz-Jaskolska, M. \& Bidzinski, M. Laparoscopic sentinel lymph node mapping after cervical injection of indocyanine green for endometrial cancer - preliminary report. Wideochir Inne Tech Maloinwazyjne 10, 406-412 (2015).

210. Chen, C.H., Chen, H.H. \& Liu, W.M. Detection of Sentinel Lymph Node Mapping Using Indocyanine Green in the Management of Endometrial Cancer: A Pilot Study. J Minim Invasive Gynecol 22, S239 (2015).

211. Plante, M., et al. Sentinel node mapping with indocyanine green and endoscopic near-infrared fluorescence imaging in endometrial cancer. A pilot study and review of the literature. Gynecol Oncol 137, 443-447 (2015).

212. Sinno, A.K., Fader, A.N., Roche, K.L., Giuntoli, R.L., 2nd \& Tanner, E.J. A comparison of colorimetric versus fluorometric sentinel lymph node mapping during robotic surgery for endometrial cancer. Gynecol Oncol 134, 281-286 (2014).

213. Blakely, M., et al. Sentinel Lymph Node Ultra-staging as a Supplement for Endometrial Cancer Intraoperative Frozen Section Deficiencies. Int J Gynecol Pathol 38, 52-58 (2019).

214. Multinu, F., et al. Ultrastaging of negative pelvic lymph nodes to decrease the true prevalence of isolated paraaortic dissemination in endometrial cancer. Gynecol Oncol 154, 60-64 (2019).

215. Gorostidi, M., et al. Maximizing Sentinel Lymph Node Detection: Aortic Sentinel Lymph Node Detection in Endometrial Cancer. J Minim Invasive Gynecol 26, 2324 (2019).

216. Taskin, S., Altin, D., Sukur, Y.E. \& Ortac, F. Extrapelvic Sentinel Lymph Nodes in Endometrial Cancer Patients With Unmapped Pelvic Side: A Brief Report. Int J Gynecol Cancer 28, 700-703 (2018).

217. Fernandez-Prada, S., Delgado-Sanchez, E., De Santiago, J. \& Zapardiel, I. Laparoscopic Sentinel Node Biopsy Using Real-time 3-dimensional Singlephoton Emission Computed Tomographic Guidance in Endometrial Cancer. J Minim Invasive Gynecol 22, 1075-1078 (2015).

218. Ruiz, R., et al. Sentinel Node Biopsy in Endometrial Cancer With Dual Cervical and Fundal Indocyanine Green Injection. Int J Gynecol Cancer 28, 139-144 (2018).

219. Euscher, E., et al. Ultrastaging of Sentinel Lymph Nodes in Endometrial Carcinoma According to Use of 2 Different Methods. Int J Gynecol Pathol 37, 242-251 (2018).

220. Schlappe, B.A., et al. Multicenter study comparing oncologic outcomes between two nodal assessment methods in patients with deeply invasive endometrioid endometrial carcinoma: A sentinel lymph node algorithm versus a comprehensive pelvic and paraaortic lymphadenectomy. Gynecol Oncol 151, 235-242 (2018).

221. Buda, A., et al. The impact of the type of nodal assessment on prognosis in patients with high-intermediate and high-risk ESMO/ESGO/ESTRO group endometrial cancer. A multicenter Italian study. Eur J Surg Oncol 44, 15621567 (2018). 
222. Mendivil, A.A., et al. The safety and feasibility of minimally invasive sentinel lymph node staging using indocyanine green in the management of endometrial cancer. Eur J Obstet Gynecol Reprod Biol 224, 29-32 (2018).

223. Restaino, S., et al. Role of blue dye for sentinel lymph node detection in early endometrial cancer. Gynecol Surg 14, 23 (2017).

224. Sinno, A.K., et al. Reducing overtreatment: A comparison of lymph node assessment strategies for endometrial cancer. Gynecol Oncol 143, 281-286 (2016).

225. Naoura, I., Canlorbe, G., Bendifallah, S., Ballester, M. \& Darai, E. Relevance of sentinel lymph node procedure for patients with high-risk endometrial cancer. Gynecol Oncol 136, 60-64 (2015).

226. Papadia, A., et al. Retrospective validation of the laparoscopic ICG SLN mapping in patients with grade 3 endometrial cancer. J Cancer Res Clin Oncol 144, 1385-1393 (2018).

227. Papadia, A., et al. FIGO stage IIIC endometrial cancer identification among patients with complex atypical hyperplasia, grade 1 and 2 endometrioid endometrial cancer: laparoscopic indocyanine green sentinel lymph node mapping versus frozen section of the uterus, why get around the problem? J Cancer Res Clin Oncol 143, 491-497 (2017).

228. Ghezzi, F., Casarin, J. \& Uccella, S. Minilaparoscopic Sentinel Node Detection in Endometrial Cancer: Further Reducing Invasiveness for Patients with EarlyStage Disease. Ann Surg Oncol 22 Suppl 3, S342 (2015).

229. Montero Macias, R., et al. Precaval positive sentinel lymph node with bilateral negative pelvic sentinel lymph node in low-risk endometrial cancer patient. J Gynecol Obstet Hum Reprod (2018).

230. Brugger, S., et al. Endometrial cancerhow many patients could benefit from sentinel lymph node dissection? World J Surg Oncol 16, 95 (2018).

231. Kataoka, F., et al. The importance of para-aortic lymph nodes in sentinel lymph node mapping for endometrial cancer by using hysteroscopic radioisotope tracer injection combined with subserosal dye injection: Prospective study. Gynecol Oncol 140, 400-404 (2016).

232. Backes, F.J., et al. Prospective clinical trial of robotic sentinel lymph node assessment with isosulfane blue (ISB) and indocyanine green (ICG) in endometrial cancer and the impact of ultrastaging (NCT01818739). Gynecol Oncol 153, 496-499 (2019).

233. Togami, S., et al. Prospective study of sentinel lymph node mapping for endometrial cancer. Int $J$ Gynaecol Obstet 143, 313-318 (2018).

234. Rajanbabu, A. \& Agarwal, R. A prospective evaluation of the sentinel node mapping algorithm in endometrial cancer and correlation of its performance against endometrial cancer risk subtypes. Eur J Obstet Gynecol Reprod Biol 224, 77-80 (2018).

235. Farzaneh, F., et al. Value of Sentinel Lymph Node (SLN) Mapping and Biopsy using Combined Intracervical Radiotracers and Blue Dye Injections for Endometrial Cancer. Asian Pac J Cancer Prev 18, 431-435 (2017).

236. Holloway, R.W., et al. A Prospective Cohort Study Comparing Colorimetric and Fluorescent Imaging for Sentinel Lymph Node Mapping in Endometrial Cancer. Ann Surg Oncol 24, 1972-1979 (2017).

237. Soliman, P.T., et al. A prospective validation study of sentinel lymph node 
mapping for high-risk endometrial cancer. Gynecol Oncol 146, 234-239 (2017).

238. Frati, A., et al. Contribution of Lymphoscintigraphy for Sentinel Lymph Node Biopsy in Women with Early Stage Endometrial Cancer: Results of the SENTI-ENDO Study. Ann Surg Oncol 22, 1980-1986 (2015).

239. Hagen, B., et al. Indocyanine green fluorescence imaging of lymph nodes during robotic-assisted laparoscopic operation for endometrial cancer. A prospective validation study using a sentinel lymph node surgical algorithm. Gynecol Oncol 143, 479-483 (2016).

240. Geppert, B., Lonnerfors, C., Bollino, M. \& Persson, J. Sentinel lymph node biopsy in endometrial cancer-Feasibility, safety and lymphatic complications. Gynecol Oncol 148, 491-498 (2018).

241. Zuo, J., et al. Comparison Study of Laparoscopic Sentinel Lymph Node Mapping in Endometrial Carcinoma Using Carbon Nanoparticles and Lymphatic Pathway Verification. J Minim Invasive Gynecol (2018).

242. Accorsi, G.S., et al. Sentinel Lymph Node Mapping vs Systematic Lymphadenectomy for Endometrial Cancer: Surgical Morbidity and Lymphatic Complications. J Minim Invasive Gynecol (2019).

243. Kim, C.H., et al. Sentinel lymph node mapping with pathologic ultrastaging: a valuable tool for assessing nodal metastasis in low-grade endometrial cancer with superficial myoinvasion. Gynecol Oncol 131, 714-719 (2013).

244. Rozenholc, A., et al. Green versus blue: Randomized controlled trial comparing indocyanine green with methylene blue for sentinel lymph node detection in endometrial cancer. Gynecol Oncol 153, 500-504 (2019).
245. Kang, S., et al. Sentinel lymph node biopsy in endometrial cancer: metaanalysis of 26 studies. Gynecol Oncol 123, 522-527 (2011).

246. Frumovitz, M., et al. Near-infrared fluorescence for detection of sentinel lymph nodes in women with cervical and uterine cancers (FILM): a randomised, phase 3 , multicentre, noninferiority trial. Lancet Oncol 19, 13941403 (2018).

247. Ignatov, A., et al. Lymph node micrometastases and outcome of endometrial cancer. Gynecol Oncol 154, 475-479 (2019).

248. Kitchener, H., Swart, A.M., Qian, Q., Amos, C. \& Parmar, M.K. Efficacy of systematic pelvic lymphadenectomy in endometrial cancer (MRC ASTEC trial): a randomised study. Lancet 373, 125136 (2009).

249. Benedetti Panici, P., et al. Systematic pelvic lymphadenectomy vs. no lymphadenectomy in early-stage endometrial carcinoma: randomized clinical trial. J Natl Cancer Inst 100, 1707-1716 (2008).

250. Gu, H., et al. Survival Impact of Ovarian Preservation on Women With EarlyStage Endometrial Cancer: A Systematic Review and Meta-analysis. Int J Gynecol Cancer 27, 77-84 (2017).

251. Lyu, T., et al. Ovarian preservation for premenopausal women with early-stage endometrial cancer: a Chinese retrospective study. J Int Med Res, 300060518822432 (2019).

252. Lau, H.Y., et al. Outcome of ovarian preservation during surgical treatment for endometrial cancer: A Taiwanese Gynecologic Oncology Group study. Taiwan J Obstet Gynecol 54, 532-536 (2015).

253. Kinjyo, Y., et al. Ovarian preservation in young women with endometrial cancer 
of endometrioid histology. Acta Obstet Gynecol Scand 94, 430-434 (2015).

254. Anggraeni, T.D., Al Fattah, A.N. \& Surya, R. Prophylactic salpingectomy and ovarian cancer: An evidence-based analysis. South Asian J Cancer 7, 42-45 (2018).

255. Peccatori, F.A., et al. Fertility preservation in women harboring deleterious BRCA mutations: ready for prime time? Hum Reprod 33, 181-187 (2018).

256. Liu, $\mathrm{T}$., et al. Impact of Radical Hysterectomy Versus Simple Hysterectomy on Survival of Patients with Stage 2 Endometrial Cancer: A Meta-analysis. Ann Surg Oncol (2019).

257. van der Steen-Banasik, E., et al. Systemic review: Radiation therapy alone in medical non-operable endometrial carcinoma. Eur J Cancer 65, 172-181 (2016).

258. Dutta, S.W., Trifiletti, D.M., Grover, S., Boimel, P. \& Showalter, T.N. Management of elderly patients with early-stage medically inoperable endometrial cancer: Systematic review and National Cancer Database analysis. Brachytherapy 16, 526-533 (2017).

259. Acharya, S., et al. Brachytherapy Is Associated With Improved Survival in Inoperable Stage I Endometrial Adenocarcinoma: A Population-Based Analysis. Int J Radiat Oncol Biol Phys 93, 649-657 (2015).

260. Schwarz, J.K., et al. Consensus statement for brachytherapy for the treatment of medically inoperable endometrial cancer. Brachytherapy 14, 587-599 (2015).

261. Gill, B.S., et al. Image-based threedimensional conformal brachytherapy for medically inoperable endometrial carcinoma. Brachytherapy 13, 542-547 (2014).
262. Yang, B., et al. Treatment efficiency of comprehensive hysteroscopic evaluation and lesion resection combined with progestin therapy in young women with endometrial atypical hyperplasia and endometrial cancer. Gynecol Oncol 153, 55-62 (2019).

263. Chae, S.H., et al. Pregnancy and oncologic outcomes after fertilitysparing management for early stage endometrioid endometrial cancer. Int $J$ Gynecol Cancer 29, 77-85 (2019).

264. Giampaolino, P., et al. Hysteroscopic Endometrial Focal Resection followed by Levonorgestrel Intrauterine Device Insertion as a Fertility-Sparing Treatment of Atypical Endometrial Hyperplasia and Early Endometrial Cancer: A Retrospective Study. J Minim Invasive Gynecol (2018).

265. Tamauchi, S., et al. Efficacy of medroxyprogesterone acetate treatment and retreatment for atypical endometrial hyperplasia and endometrial cancer. J Obstet Gynaecol Res 44, 151-156 (2018).

266. Kim, S.R., et al. Fertility-Sparing Management Using Progestin for Young Women with Endometrial Cancer From a Population-Based Study. J Obstet Gynaecol Can 40, 328-333 (2018).

267. Yamagami, W., et al. Is repeated highdose medroxyprogesterone acetate (MPA) therapy permissible for patients with early stage endometrial cancer or atypical endometrial hyperplasia who desire preserving fertility? J Gynecol Oncol 29, e21 (2018).

268. Fan, Z., et al. Fertility-Preserving Treatment in Young Women With Grade 1 Presumed Stage IA Endometrial Adenocarcinoma: A Meta-Analysis. Int J Gynecol Cancer 28, 385-393 (2018).

269. Hwang, J.Y., et al. Combined Oral Medroxyprogesterone/LevonorgestrelIntrauterine System Treatment for 
Women With Grade 2 Stage IA Endometrial Cancer. Int J Gynecol Cancer 27, 738-742 (2017).

270. Di Spiezio Sardo, A., et al. Should endometrial biopsy under direct hysteroscopic visualization using the grasp technique become the new gold standard for the preoperative evaluation of the patient with endometrial cancer? Gynecol Oncol 158, 347-353 (2020).

271. Lago, V., Martin, B., Ballesteros, E., Cardenas-Rebollo, J.M. \& Minig, L. Tumor Grade Correlation Between Preoperative Biopsy and Final Surgical Specimen in Endometrial Cancer: The Use of Different Diagnostic Methods and Analysis of Associated Factors. Int $J$ Gynecol Cancer 28, 1258-1263 (2018).

272. Larish, A., Kumar, A., Weaver, A. \& Mariani, A. Impact of hysteroscopy on course of disease in high-risk endometrial carcinoma. Int $J$ Gynecol Cancer (2020).

273. Minimum training recommendations for the practice of medical ultrasound. Ultraschall Med 27, 79-105 (2006).

274. Kitson, S., et al. Interventions for weight reduction in obesity to improve survival in women with endometrial cancer. Cochrane Database Syst Rev 2, CD012513 (2018).

275. Raffone, A., et al. Diabetes mellitus and responsiveness of endometrial hyperplasia and early endometrial cancer to conservative treatment. Gynecol Endocrinol 35, 932-937 (2019).

276. Chu, D., et al. Effect of metformin use on the risk and prognosis of endometrial cancer: a systematic review and metaanalysis. BMC Cancer 18, 438 (2018).

277. Greenwald, Z.R., Huang, L.N., Wissing, M.D., Franco, E.L. \& Gotlieb, W.H. Does hormonal therapy for fertility preservation affect the survival of young women with early-stage endometrial cancer? Cancer 123, 1545-1554 (2017).

278. Wei, J., Zhang, W., Feng, L. \& Gao, W. Comparison of fertility-sparing treatments in patients with early endometrial cancer and atypical complex hyperplasia: A meta-analysis and systematic review. Medicine (Baltimore) 96, e8034 (2017).

279. Zhang, Q., et al. Comparison among fertility-sparing therapies for well differentiated early-stage endometrial carcinoma and complex atypical hyperplasia. Oncotarget 8, 57642-57653 (2017).

280. Zapardiel, I., Cruz, M., Diestro, M.D., Requena, A. \& Garcia-Velasco, J.A. Assisted reproductive techniques after fertility-sparing treatments in gynaecological cancers. Hum Reprod Update 22, 281-305 (2016).

281. Marton, I., et al. Two cases of successful pregnancies after hysteroscopic removal of endometrioid adenocarcinoma grade I, stage IA, in young women with Lynch syndrome. $J$ Turk Ger Gynecol Assoc 15, 63-66 (2014).

282. Casadio, P., et al. Conservative hysteroscopic treatment of stage I well differentiated endometrial cancer in patients with high surgical risk: a pilot study. J Gynecol Oncol 30, e62 (2019).

283. Yang, H.C., Liu, J.C. \& Liu, F.S. Fertilitypreserving treatment of stage IA, welldifferentiated endometrial carcinoma in young women with hysteroscopic resection and high-dose progesterone therapy. Taiwan J Obstet Gynecol 58, 9093 (2019).

284. Arendas, K., Aldossary, M., Cipolla, A., Leader, A. \& Leyland, N.A. Hysteroscopic resection in the management of earlystage endometrial cancer: report of 2 cases and review of the literature. $J$ 
Minim Invasive Gynecol 22, 34-39 (2015).

285. Casadio, P., et al. Fertility-Sparing Treatment of Endometrial Cancer with Initial Infiltration of Myometrium by Resectoscopic Surgery: A Pilot Study. Oncologist 23, 478-480 (2018).

286. Leone Roberti Maggiore, U., et al. Efficacy and fertility outcomes of levonorgestrel-releasing intra-uterine system treatment for patients with atypical complex hyperplasia or endometrial cancer: a retrospective study. J Gynecol Oncol 30, e57 (2019).

287. Pal, N., et al. Treatment of Low-Risk Endometrial Cancer and Complex Atypical Hyperplasia With the Levonorgestrel-Releasing Intrauterine Device. Obstet Gynecol 131, 109-116 (2018).

288. Sletten, E.T., Arnes, M., Vereide, A.B. \& Orbo, A. Low-dose LNG-IUS as Therapy for Endometrial Hyperplasia. A Prospective Cohort Pilot Study. Anticancer Res 38, 2883-2889 (2018).

289. Orbo, A., Vereide, A., Arnes, M., Pettersen, I. \& Straume, B. Levonorgestrel-impregnated intrauterine device as treatment for endometrial hyperplasia: a national multicentre randomised trial. BJOG 121, 477-486 (2014).

290. Luo, L., et al. Oral and intrauterine progestogens for atypical endometrial hyperplasia. Cochrane Database Syst Rev 12, CD009458 (2018).

291. Marnach, M.L., et al. Oral Progestogens Versus Levonorgestrel-Releasing Intrauterine System for Treatment of Endometrial Intraepithelial Neoplasia<sup/>. J Womens Health (Larchmt) 26, 368-373 (2017).

292. Kim, M.K., et al. Six months response rate of combined oral medroxyprogesterone/levonorgestrelintrauterine system for early-stage endometrial cancer in young women: a Korean Gynecologic-Oncology Group Study. J Gynecol Oncol 30, e47 (2019).

293. Tock, S., et al. Fertility Sparing Treatment in Patients With Early Stage Endometrial Cancer, Using a Combination of Surgery and GnRH Agonist: A Monocentric Retrospective Study and Review of the Literature. Front Med (Lausanne) 5, 240 (2018).

294. Jadoul, P. \& Donnez, J. Conservative treatment may be beneficial for young women with atypical endometrial hyperplasia or endometrial adenocarcinoma. Fertil Steril 80, 13151324 (2003).

295. Stewart, C.J.R., et al. Guidelines to Aid in the Distinction of Endometrial and Endocervical Carcinomas, and the Distinction of Independent Primary Carcinomas of the Endometrium and Adnexa From Metastatic Spread Between These and Other Sites. Int J Gynecol Pathol 38 Suppl 1, S75-S92 (2019).

296. Connell, P.P., Rotmensch, J., Waggoner, S. \& Mundt, A.J. The significance of adnexal involvement in endometrial carcinoma. Gynecol Oncol 74, 74-79 (1999).

297. Soliman, P.T., et al. Synchronous primary cancers of the endometrium and ovary: a single institution review of 84 cases. Gynecol Oncol 94, 456-462 (2004)

298. Schultheis, A.M., et al. Massively Parallel Sequencing-Based Clonality Analysis of Synchronous Endometrioid Endometrial and Ovarian Carcinomas. J Natl Cancer Inst 108, djv427 (2016).

299. Anglesio, M.S., et al. Synchronous Endometrial and Ovarian Carcinomas: Evidence of Clonality. J Natl Cancer Inst 108, djv428 (2016).

300. Turashvili, G., et al. Risk-based stratification of carcinomas concurrently involving the 
endometrium and ovary. Gynecol Oncol 152, 38-45 (2019).

301. Keys, H.M., et al. A phase III trial of surgery with or without adjunctive external pelvic radiation therapy in intermediate risk endometrial adenocarcinoma: a Gynecologic Oncology Group study. Gynecol Oncol 92, 744-751 (2004).

302. Creutzberg, C.L., et al. Surgery and postoperative radiotherapy versus surgery alone for patients with stage-1 endometrial carcinoma: multicentre randomised trial. PORTEC Study Group. Post Operative Radiation Therapy in Endometrial Carcinoma. Lancet 355, 1404-1411 (2000).

303. Aalders, J., Abeler, V., Kolstad, P. \& Onsrud, M. Postoperative external irradiation and prognostic parameters in stage I endometrial carcinoma: clinical and histopathologic study of 540 patients. Obstet Gynecol 56, 419-427 (1980).

304. Blake, P., et al. Adjuvant external beam radiotherapy in the treatment of endometrial cancer (MRC ASTEC and NCIC CTG EN.5 randomised trials): pooled trial results, systematic review, and meta-analysis. Lancet 373, 137-146 (2009).

305. Barney, B.M., et al. The role of vaginal brachytherapy in the treatment of surgical stage I papillary serous or clear cell endometrial cancer. Int $J$ Radiat Oncol Biol Phys 85, 109-115 (2013).

306. Wortman, B.G., et al. Ten-year results of the PORTEC-2 trial for highintermediate risk endometrial carcinoma: improving patient selection for adjuvant therapy. $\mathrm{Br} J$ Cancer 119, 1067-1074 (2018).

307. Nout, R.A., et al. Vaginal brachytherapy versus pelvic external beam radiotherapy for patients with endometrial cancer of high- intermediate risk (PORTEC-2): an openlabel, non-inferiority, randomised trial. Lancet 375, 816-823 (2010).

308. Sunil, R.A., et al. Combined external beam radiotherapy and vaginal brachytherapy versus vaginal brachytherapy in stage I, intermediateand high-risk cases of endometrium carcinoma. J Contemp Brachytherapy 10, 105-114 (2018).

309. Cham, S., et al. Utility of radiation therapy for early-stage uterine papillary serous carcinoma. Gynecol Oncol 145, 269-276 (2017).

310. Shinde, A., et al. Improved survival with adjuvant brachytherapy in stage IA endometrial cancer of unfavorable histology. Gynecol Oncol 151, 82-90 (2018).

311. Qu, X.M., et al. The role of adjuvant therapy in stage IA serous and clear cell uterine cancer: A multi-institutional pooled analysis. Gynecol Oncol 149, 283290 (2018).

312. Donovan, E., et al. Outcomes of Adjuvant Therapy for Stage IA Serous Endometrial Cancer. Cureus 10, e3387 (2018).

313. Sorbe, B., et al. External pelvic and vaginal irradiation versus vaginal irradiation alone as postoperative therapy in medium-risk endometrial carcinoma--a prospective randomized study. Int J Radiat Oncol Biol Phys 82, 1249-1255 (2012).

314. Ortoft, G., Hansen, E.S. \& Bertelsen, K. Omitting adjuvant radiotherapy in endometrial cancer increases the rate of locoregional recurrences but has no effect on long-term survival: the Danish Endometrial Cancer Study. Int J Gynecol Cancer 23, 1429-1437 (2013).

315. Randall, M.E., et al. Phase III Trial: Adjuvant Pelvic Radiation Therapy Versus Vaginal Brachytherapy Plus Paclitaxel/Carboplatin in High- 
Intermediate and High-Risk Early Stage Endometrial Cancer. J Clin Oncol 37, 1810-1818 (2019).

316. Maggi, R., et al. Adjuvant chemotherapy vs radiotherapy in high-risk endometrial carcinoma: results of a randomised trial. $\mathrm{Br} J$ Cancer 95, 266271 (2006).

317. Susumu, N., et al. Randomized phase III trial of pelvic radiotherapy versus cisplatin-based combined chemotherapy in patients with intermediate- and high-risk endometrial cancer: a Japanese Gynecologic Oncology Group study. Gynecol Oncol 108, 226-233 (2008).

318. Hogberg, T., et al. Sequential adjuvant chemotherapy and radiotherapy in endometrial cancer--results from two randomised studies. Eur J Cancer 46, 2422-2431 (2010).

319. de Boer, S.M., et al. Adjuvant chemoradiotherapy versus radiotherapy alone for women with high-risk endometrial cancer (PORTEC3): final results of an international, open-label, multicentre, randomised, phase 3 trial. Lancet Oncol 19, 295-309 (2018).

320. Leon-Castillo, A., et al. Molecular Classification of the PORTEC-3 Trial for High-Risk Endometrial Cancer: Impact on Prognosis and Benefit From Adjuvant Therapy. J Clin Oncol 38, 3388-3397 (2020).

321. Randall, M., et al. A phase III trial of pelvic radiation therapy versus vaginal cuff brachytherapy followed by paclitaxel/carboplatin chemotherapy in patients with high-risk, early-stage endometrial cancer: a gynecology oncology group study. Int J Radiat Oncol Biol Phys 99, 1313 (2017).

322. de Boer, S.M., et al. Adjuvant chemoradiotherapy versus radiotherapy alone in women with high- risk endometrial cancer (PORTEC-3): patterns of recurrence and post-hoc survival analysis of a randomised phase 3 trial. Lancet Oncol 20, 1273-1285 (2019).

323. Matei, D., et al. Adjuvant Chemotherapy plus Radiation for Locally Advanced Endometrial Cancer. N Engl J Med 380, 2317-2326 (2019).

324. Randall, M.E., et al. Phase III Trial: Adjuvant Pelvic Radiation Therapy Versus Vaginal Brachytherapy Plus Paclitaxel/Carboplatin in HighIntermediate and High-Risk Early Stage Endometrial Cancer. J Clin Oncol, JC01801575 (2019).

325. Albeesh, R., et al. Adjuvant therapy in stage III endometrial cancer confined to the pelvis. Gynecol Oncol 152, 26-30 (2019).

326. Onal, C., et al. Treatment outcomes of endometrial cancer patients with paraaortic lymph node metastasis: a multi-institutional analysis. Int J Gynecol Cancer 29, 94-101 (2019).

327. Scharl, S., et al. Does post-operative radiochemotherapy improve survival in high-grade endometrial cancer patients? Results of a population-based cohort analysis of a cancer registry. Arch Gynecol Obstet 297, 1245-1253 (2018).

328. Rodrigues da Cunha Colombo Bonadio, R., et al. Adjuvant Carboplatin and Paclitaxel Chemotherapy Followed by Radiotherapy in High-Risk Endometrial Cancer: A Retrospective Analysis. J Glob Oncol 4, 1-8 (2018).

329. Chapman, B.V., et al. Adjuvant combined-modality therapy for stage IIIC endometrioid and nonendometrioid endometrial cancer. Gynecol Oncol (2019).

330. Binder, P.S., et al. Benefit of combination chemotherapy and radiation stratified by grade of stage IIIC endometrial 
cancer. Gynecol Oncol 147, 309-314 (2017).

331. Lee, J.K., Mahan, M., Hanna, R.K. \& Elshaikh, M.A. Survival outcomes and patterns of failure in women with stage IIIC2 endometrial carcinoma. Eur $J$ Obstet Gynecol Reprod Biol 216, 192-197 (2017).

332. Signorelli, M., et al. Adjuvant sequential chemo and radiotherapy improves the oncological outcome in high risk endometrial cancer. J Gynecol Oncol 26, 284-292 (2015).

333. Bogani, G., et al. Chemotherapy reduces para-aortic node recurrences in endometrial cancer with positive pelvic and unknown para-aortic nodes. Int $J$ Gynecol Cancer 25, 263-268 (2015).

334. Lee, L.J., Bu, P., Feltmate, C. \& Viswanathan, A.N. Adjuvant chemotherapy with external beamradiation therapy for high-grade, node-positive endometrial cancer. Int J Gynecol Cancer 24, 1441-1448 (2014).

335. Bakkum-Gamez, J.N., et al. Efficacy of contemporary chemotherapy in stage IIIC endometrial cancer: a histologic dichotomy. Gynecol Oncol 132, 578-584 (2014).

336. Xiang, M., English, D.P. \& Kidd, E.A. National patterns of care and cancerspecific outcomes of adjuvant treatment in patients with serous and clear cell endometrial carcinoma. Gynecol Oncol 152, 599-604 (2019).

337. Holloway, C.L., Alexander, C., Walter, C., Aquino-Parsons, C. \& Truong, P.T. Stage IIIC Endometrial Cancer: Relapse and Survival Outcomes in Women Treated With Pelvic or Extended Field ParaAortic Nodal Radiation Therapy. Am J Clin Oncol 40, 458-463 (2017).

338. Chen, J.R., et al. Outcomes of Patients With Surgically and Pathologically Staged IIIA-IVB Pure Endometrioid-type Endometrial Cancer: A Taiwanese
Gynecology Oncology Group (TGOG2005) Retrospective Cohort Study (A STROBE-Compliant Article). Medicine (Baltimore) 95, e3330 (2016).

339. Fleming, N.D., et al. Impact of Lymph Node Ratio and Adjuvant Therapy in Node-Positive Endometrioid Endometrial Cancer. Int J Gynecol Cancer 25, 1437-1444 (2015).

340. Boothe, D., et al. Chemoradiation versus chemotherapy or radiation alone in stage III endometrial cancer: Patterns of care and impact on overall survival. Gynecol Oncol 141, 421-427 (2016).

341. Boothe, D., et al. Corrigendum to "Chemoradiation versus chemotherapy or radiation alone in stage III endometrial cancer: Patterns of care and impact on overall survival" [Gynecol. Oncol. 141 (2016) 421-427]. Gynecol Oncol 143, 690-691 (2016).

342. Wong, A.T., et al. Utilization of adjuvant therapies and their impact on survival for women with stage IIIC endometrial adenocarcinoma. Gynecol Oncol 142, 514-519 (2016).

343. Lin, J.F., et al. Survival advantage associated with multimodal therapy in women with node-positive (stage-IIIC) uterine papillary serous carcinoma: a National Cancer Database study. BJOG 123, 1846-1852 (2016).

344. Barlin, J.N., Puri, I. \& Bristow, R.E. Cytoreductive surgery for advanced or recurrent endometrial cancer: a metaanalysis. Gynecol Oncol 118, 14-18 (2010).

345. Rajkumar, S., et al. Advanced stage (IIIC/IV) endometrial cancer: Role of cytoreduction and determinants of survival. Eur J Obstet Gynecol Reprod Biol 234, 26-31 (2019).

346. Solmaz, U., et al. Stage-III and -IV endometrial cancer: A single oncology centre review of 104 cases. J Obstet Gynaecol 36, 81-86 (2016). 
347. Cirik, D.A., et al. Stage IVB endometrial cancer confined to the abdomen: is chemotherapy superior to radiotherapy? Eur J Gynaecol Oncol 37, 226-231 (2016).

348. Schmidt, A.M., Imesch, P., Fink, D. \& Egger, H. Pelvic Exenterations for Advanced and Recurrent Endometrial Cancer: Clinical Outcomes of 40 Patients. Int J Gynecol Cancer 26, 716721 (2016).

349. Vitale, S.G., Valenti, G., Gulino, F.A., Cignini, P. \& Biondi, A. Surgical treatment of high stage endometrial cancer: current perspectives. Updates Surg 68, 149-154 (2016).

350. Tangjitgamol, S., Kittisiam, T. \& Sriraumpuch, J. Impact of Metastatic Lymph Node to Total Lymph Node Ratio on Survival of Endometrial Cancer Patients. Gynecol Obstet Invest 84, 463471 (2019).

351. Yoon, M.S., et al. Impact of paraaortic lymphadenectomy for endometrial cancer with positive pelvic lymph nodes: A Korean Radiation Oncology Group study (KROG 13-17). Eur J Surg Oncol 42, 1497-1505 (2016).

352. Bogani, G., et al. Neoadjuvant chemotherapy followed by interval debulking surgery for unresectable stage IVB Serous endometrial cancer. Tumori 105, 92-97 (2019).

353. Boisen, M.M., et al. Surgical Outcomes of Patients Undergoing Extrafascial Hysterectomy After Neoadjuvant Radiotherapy With or Without Chemotherapy for Locally Advanced Endometrial Cancer Clinically Extending to the Cervix or Parametria. Int J Gynecol Cancer 27, 1149-1154 (2017).

354. de Lange, N.M., et al. Neoadjuvant chemotherapy followed by surgery for advanced-stage endometrial cancer. Curr Oncol 26, e226-e232 (2019).
355. Iheagwara, U.K., et al. Neoadjuvant Chemoradiation Therapy Followed by Extrafascial Hysterectomy in Locally Advanced Type II Endometrial Cancer Clinically Extending to Cervix. Pract Radiat Oncol 9, 248-256 (2019).

356. Khouri, O.R., et al. Neoadjuvant chemotherapy in patients with advanced endometrial cancer. Cancer Chemother Pharmacol 84, 281-285 (2019).

357. Palisoul, M. \& Mutch, D.G. The clinical management of inoperable endometrial carcinoma. Expert Rev Anticancer Ther 16, 515-521 (2016).

358. Rabinovich, A. Neo-adjuvant chemotherapy for advanced stage endometrial carcinoma: a glimmer of hope in select patients. Arch Gynecol Obstet 293, 47-53 (2016).

359. Vandenput, I., et al. Neoadjuvant chemotherapy followed by interval debulking surgery in patients with serous endometrial cancer with transperitoneal spread (stage IV): a new preferred treatment? Br J Cancer 101, 244-249 (2009).

360. Conway, J.L., Lukovic, J., Laframboise, S., Ferguson, S.E. \& Han, K. Brachy-ing Unresectable Endometrial Cancers with Magnetic Resonance Guidance. Cureus 10, e2274 (2018).

361. Townamchai, K., et al. Radiation dose escalation using intensity modulated radiation therapy for gross unresected node-positive endometrial cancer. Pract Radiat Oncol 4, 90-98 (2014).

362. Francis, S.R., et al. Recurrent early stage endometrial cancer: Patterns of recurrence and results of salvage therapy. Gynecol Oncol (2019).

363. Hardarson, H.A., Heidemann, L.N., dePont Christensen, R., Mogensen, O. \& Jochumsen, K.M. Vaginal vault recurrences of endometrial cancer in non-irradiated patients - Radiotherapy 
or surgery. Gynecol Oncol Rep 11, 26-30 (2015).

364. Shikama, A., et al. Predictors of favorable survival after secondary cytoreductive surgery for recurrent endometrial cancer. Int J Clin Oncol (2019).

365. Turan, T., et al. Salvage Cytoreductive Surgery for Recurrent Endometrial Cancer. Int J Gynecol Cancer 25, 16231632 (2015).

366. Ren, Y., Shan, B., Shi, D. \& Wang, H. Salvage cytoreductive surgery for patients with recurrent endometrial cancer: a retrospective study. $B M C$ Cancer 14, 135 (2014).

367. Papadia, A., et al. Surgical Treatment of Recurrent Endometrial Cancer: Time for a Paradigm Shift. Ann Surg Oncol 22, 4204-4210 (2015).

368. Domenici, L., et al. Surgery for Recurrent Uterine Cancer: Surgical Outcomes and Implications for Survival-A Case Series. Int J Gynecol Cancer 27, 759-767 (2017).

369. Baek, S., et al. Salvage high-dose-rate brachytherapy for isolated vaginal recurrence of endometrial cancer. Brachytherapy 15, 812-816 (2016).

370. Chapman, C.H., Maghsoudi, K., Littell, R.D., Chen, L.M. \& Hsu, I.C. Salvage highdose-rate brachytherapy and external beam radiotherapy for isolated vaginal recurrences of endometrial cancer with no prior adjuvant therapy. Brachytherapy 16, 1152-1158 (2017).

371. Fokdal, L., et al. Toward fourdimensional image-guided adaptive brachytherapy in locally recurrent endometrial cancer. Brachytherapy 13, 554-561 (2014).

372. Ho, J.C., et al. Management of nodal recurrences of endometrial cancer with IMRT. Gynecol Oncol 139, 40-46 (2015).
373. Huang, K., et al. High-dose-rate interstitial brachytherapy for the treatment of high-volume locally recurrent endometrial carcinoma. Brachytherapy 15, 543-548 (2016).

374. Kamran, S.C., et al. MR- versus CT-based high-dose-rate interstitial brachytherapy for vaginal recurrence of endometrial cancer. Brachytherapy 16, 1159-1168 (2017).

375. Sekii, S., et al. Outcomes of salvage highdose-rate brachytherapy with or without external beam radiotherapy for isolated vaginal recurrence of endometrial cancer. J Contemp Brachytherapy 9, 209-215 (2017).

376. Vargo, J.A., et al. Definitive salvage for vaginal recurrence of endometrial cancer: the impact of modern intensitymodulated-radiotherapy with imagebased HDR brachytherapy and the interplay of the PORTEC 1 risk stratification. Radiother Oncol 113, 126131 (2014).

377. Viswanathan, A.N., et al. A prospective feasibility study of radiation and concurrent bevacizumab for recurrent endometrial cancer. Gynecol Oncol 132, 55-60 (2014).

378. Yanazume, S., Arimura, T., Kobayashi, H. \& Douchi, T. Potential proton beam therapy for recurrent endometrial cancer in the vagina. $J$ Obstet Gynaecol Res 41, 813-816 (2015).

379. Chiantera, V., et al. Pelvic exenteration for recurrent endometrial adenocarcinoma: a retrospective multiinstitutional study about 21 patients. Int J Gynecol Cancer 24, 880-884 (2014).

380. Margolis, B., Kim, S.W. \& Chi, D.S. Longterm survival after anterior pelvic exenteration and total vaginectomy for recurrent endometrial carcinoma with metastatic inguinal nodes at the time of surgery. Gynecol Oncol Rep 19, 39-41 (2017). 
381. Ling, D.C., Vargo, J.A., Glaser, S.M., Kim, H. \& Beriwal, S. Outcomes after definitive re-irradiation with 3D brachytherapy with or without external beam radiation therapy for vaginal recurrence of endometrial cancer. Gynecol Oncol 152, 581-586 (2019).

382. Mabuchi, S., et al. Reirradiation using high-dose-rate interstitial brachytherapy for locally recurrent cervical cancer: a single institutional experience. Int J Gynecol Cancer 24, 141148 (2014).

383. Arians, N., et al. Outcome of patients with local recurrent gynecologic malignancies after resection combined with intraoperative electron radiation therapy (IOERT). Radiat Oncol 11, 44 (2016).

384. Feddock, J., et al. Reirradiation Using Permanent Interstitial Brachytherapy: A Potentially Durable Technique for Salvaging Recurrent Pelvic Malignancies. Int J Radiat Oncol Biol Phys 99, 1225-1233 (2017).

385. Wooten, C.E., et al. Implementation and early clinical results utilizing Cs-131 permanent interstitial implants for gynecologic malignancies. Gynecol Oncol 133, 268-273 (2014).

386. Widder, J. \& Lodeweges, J. Synchronous or Metachronous Oligometastases. J Thorac Oncol 12, e191-e192 (2017).

387. Xu, L. \& Burke, A.P. Pulmonary oligometastases: histological features and difficulties in determining site of origin. Int J Surg Pathol 20, 577-588 (2012).

388. Kaneda, H. \& Saito, Y. Oligometastases: Defined by prognosis and evaluated by cure. Cancer Treat Commun 3, 1-6 (2015).

389. Kunos, C.A., et al. Phase II Clinical Trial of Robotic Stereotactic Body Radiosurgery for Metastatic Gynecologic Malignancies. Front Oncol 2, 181 (2012).
390. Lodeweges, J.E., et al. Long-term Outcome of Surgery or Stereotactic Radiotherapy for Lung Oligometastases. J Thorac Oncol 12, 1442-1445 (2017).

391. Palma, D.A., et al. Stereotactic ablative radiotherapy versus standard of care palliative treatment in patients with oligometastatic cancers (SABR-COMET): a randomised, phase 2, open-label trial. Lancet 393, 2051-2058 (2019).

392. Loveman, E., et al. The clinical effectiveness and cost-effectiveness of ablative therapies in the management of liver metastases: systematic review and economic evaluation. Health Technol Assess 18, vii-viii, 1-283 (2014).

393. van Weelden, W.J., Massuger, L., Pijnenborg, J.M.A. \& Romano, A. Antiestrogen Treatment in Endometrial Cancer: A Systematic Review. Front Oncol 9, 359 (2019).

394. Ethier, J.L., Desautels, D.N., Amir, E. \& MacKay, H. Is hormonal therapy effective in advanced endometrial cancer? A systematic review and metaanalysis. Gynecol Oncol 147, 158-166 (2017).

395. Mileshkin, L., et al. Phase 2 study of anastrozole in recurrent estrogen (ER)/progesterone (PR) positive endometrial cancer: The PARAGON trial - ANZGOG 0903. Gynecol Oncol 154, 2937 (2019).

396. Slomovitz, B.M., et al. Phase II study of everolimus and letrozole in patients with recurrent endometrial carcinoma. $J$ Clin Oncol 33, 930-936 (2015).

397. Miller, D.F.V., et al. Randomized phase III non inferiority trial of first line chemotherapy for metastatic or recurrent endometrial carcinoma: a gynecologic oncology group study. [SGO abstract Late-Breaking Abstract 1]. Gynecol Oncol 125S, 771-773 (2012).

398. Rubinstein, M., et al. Retreatment with carboplatin and paclitaxel for recurrent 
endometrial cancer: A retrospective study of the Memorial Sloan Kettering Cancer Center experience. Gynecol Oncol Rep 28, 120-123 (2019).

399. Marabelle, A., et al. Efficacy of Pembrolizumab in Patients With Noncolorectal High Microsatellite Instability/Mismatch Repair-Deficient Cancer: Results From the Phase II KEYNOTE-158 Study. J Clin Oncol 38, 1$10(2020)$.

400. Mittica, G., et al. Checkpoint inhibitors in endometrial cancer: preclinical rationale and clinical activity. Oncotarget 8, 90532-90544 (2017).

401. Makker, V., et al. Lenvatinib Plus Pembrolizumab in Patients With Advanced Endometrial Cancer. J Clin Oncol, JC01902627 (2020).

402. Makker, $V_{\text {., }}$ et al. Lenvatinib plus pembrolizumab in patients with advanced endometrial cancer: an interim analysis of a multicentre, openlabel, single-arm, phase 2 trial. Lancet Oncol 20, 711-718 (2019).

403. Fader, A.N., et al. Randomized Phase II Trial of Carboplatin-Paclitaxel Versus Carboplatin-Paclitaxel-Trastuzumab in Uterine Serous Carcinomas That Overexpress Human Epidermal Growth Factor Receptor 2/neu. J Clin Oncol 36, 2044-2051 (2018).

404. Roncolato, F., Lindemann, K., Willson, M.L., Martyn, J. \& Mileshkin, L. $\mathrm{PI} 3 \mathrm{~K} / \mathrm{AKT} / \mathrm{mTOR}$ inhibitors for advanced or recurrent endometrial cancer. Cochrane Database Syst Rev 10, CD012160 (2019).

405. Coleman, R.L., et al. A phase II evaluation of selumetinib (AZD6244, ARRY-142886), a selective MEK-1/2 inhibitor in the treatment of recurrent or persistent endometrial cancer: an NRG Oncology/Gynecologic Oncology Group study. Gynecol Oncol 138, 30-35 (2015).
406. Bender, D., et al. A phase II evaluation of cediranib in the treatment of recurrent or persistent endometrial cancer: An NRG Oncology/Gynecologic Oncology Group study. Gynecol Oncol 138, 507512 (2015).

407. Dizon, D.S., et al. A phase II evaluation of nintedanib (BIBF-1120) in the treatment of recurrent or persistent endometrial cancer: an NRG Oncology/Gynecologic Oncology Group Study. Gynecol Oncol 135, 441-445 (2014).

408. Castonguay, V., et al. A phase II trial of sunitinib in women with metastatic or recurrent endometrial carcinoma: a study of the Princess Margaret, Chicago and California Consortia. Gynecol Oncol 134, 274-280 (2014).

409. Powell, M.A., et al. A phase II trial of brivanib in recurrent or persistent endometrial cancer: an NRG Oncology/Gynecologic Oncology Group Study. Gynecol Oncol 135, 38-43 (2014).

410. Oza, A.M., et al. Phase II study of temsirolimus in women with recurrent or metastatic endometrial cancer: a trial of the NCIC Clinical Trials Group. J Clin Oncol 29, 3278-3285 (2011).

411. Slomovitz, B.M., et al. A phase 2 study of the oral mammalian target of rapamycin inhibitor, everolimus, in patients with recurrent endometrial carcinoma. Cancer 116, 5415-5419 (2010).

412. Emons, G., et al. Temsirolimus in women with platinum-refractory/resistant ovarian cancer or advanced/recurrent endometrial carcinoma. A phase II study of the AGO-study group (AGO-GYN8). Gynecol Oncol 140, 450-456 (2016).

413. Tsoref, D., et al. Phase II study of oral ridaforolimus in women with recurrent or metastatic endometrial cancer. Gynecol Oncol 135, 184-189 (2014).

414. Matulonis, U., et al. Phase II study of the PI3K inhibitor pilaralisib (SAR245408; 
XL147) in patients with advanced or recurrent endometrial carcinoma. Gynecol Oncol 136, 246-253 (2015).

415. Aghajanian, C., et al. A phase II study of frontline

paclitaxel/carboplatin/bevacizumab, paclitaxel/carboplatin/temsirolimus, or ixabepilone/carboplatin/bevacizumab in advanced/recurrent endometrial cancer. Gynecol Oncol 150, 274-281 (2018).

416. Sapienza, L.G., et al. Short-course palliative radiation therapy leads to excellent bleeding control: A single centre retrospective study. Clin Transl Radiat Oncol 14, 40-46 (2019).

417. Klopp, A.H., et al. Patient-Reported Toxicity During Pelvic IntensityModulated Radiation Therapy: NRG Oncology-RTOG 1203. J Clin Oncol 36, 2538-2544 (2018).

418. Klopp, A., et al. The role of postoperative radiation therapy for endometrial cancer: Executive summary of an American Society for Radiation Oncology evidence-based guideline. Pract Radiat Oncol 4, 137-144 (2014).

419. Harkenrider, M.M., et al. American Brachytherapy Task Group Report: Adjuvant vaginal brachytherapy for early-stage endometrial cancer: A comprehensive review. Brachytherapy 16, 95-108 (2017).

420. Sturdza, A., et al. American Brachytherapy Society working group report on the patterns of care and a literature review of reirradiation for gynecologic cancers. Brachytherapy (2020).

421. Schmid, M.P., et al. Recommendations from gynaecological (GYN) GEC-ESTRO working group - ACROP: Target concept for image guided adaptive brachytherapy in primary vaginal cancer. Radiother Oncol 145, 36-44 (2020).
422. Mendez, L.C., Leung, E., Cheung, P. \& Barbera, L. The Role of Stereotactic Ablative Body Radiotherapy in Gynaecological Cancers: A Systematic Review. Clin Oncol ( $R$ Coll Radiol) 29, 378-384 (2017).

423. Hymel, R., Jones, G.C. \& Simone, C.B., 2nd. Whole pelvic intensity-modulated radiotherapy for gynecological malignancies: A review of the literature. Crit Rev Oncol Hematol 94, 371-379 (2015).

424. Kobel, M., et al. Interpretation of P53 Immunohistochemistry in Endometrial Carcinomas: Toward Increased Reproducibility. Int J Gynecol Pathol $\mathbf{3 8}$ Suppl 1, S123-S131 (2019).

425. Singh, N., et al. Pathologic Prognostic Factors in Endometrial Carcinoma (Other Than Tumor Type and Grade). Int J Gynecol Pathol 38 Suppl 1, S93S113 (2019).

426. Malpica, A., et al. Endometrial Carcinoma, Grossing and Processing Issues: Recommendations of the International Society of Gynecologic Pathologists. Int J Gynecol Pathol 38 Suppl 1, S9-S24 (2019).

427. McCluggage, W.G., et al. Data set for reporting of endometrial carcinomas: recommendations from the International Collaboration on Cancer Reporting (ICCR) between United Kingdom, United States, Canada, and Australasia. Int J Gynecol Pathol 32, 4565 (2013).

428. Soslow, R.A., et al. Endometrial Carcinoma Diagnosis: Use of FIGO Grading and Genomic Subcategories in Clinical Practice: Recommendations of the International Society of Gynecological Pathologists. Int J Gynecol Pathol 38 Suppl 1, S64-S74 (2019).

429. Weis, J. \& Hasenburg, A. Psychological support. in Textbook of Gynaecological Oncology (ed. Ayhan, A.R., N.; Gultekin, 
M.; Dursun, P.) 1495-1499 (Gunes Publishing Kopenhagen, 2016).

430. Andersen, B.L., et al. Screening, assessment, and care of anxiety and depressive symptoms in adults with cancer: an American Society of Clinical Oncology guideline adaptation. J Clin Oncol 32, 1605-1619 (2014).
431. Donovan, K.A., Grassi, L., McGinty, H.L. \& Jacobsen, P.B. Validation of the distress thermometer worldwide: state of the science. Psychooncology 23, 241-250 (2014). 


\title{
APPENDIX 1. IDENTIFICATION OF SCIENTIFIC EVIDENCE
}

\author{
Literature search in MEDLINE \\ Research period \\ 2014/01/01 - 2019/06/15
}

Indexing terms

Adjuvant chemotherapy, adjuvant radiation therapy, adjuvant radiotherapy, adjuvant treatment, advanced disease, advanced stage, adverse effect, adverse event, ALK1 inhibitor, androgen receptor, annexin 2, antiangiogenesis-based treatment, antiangiogenic therapy, antiangiogenic treatment, apitolisib, aromatase, aromatase inhibitor, asparaginase-like protein 1 , AT rich interactive domain $1 \mathrm{~A}$, atypical endometrial hyperplasia, atypical hyperplasia, aurora kinase A, autotaxin, B cell lymphoma 2, $\beta$-Catenin, B-Raf, bariatric surgery, BAX, bevacizumab, bilateral salpingo-oophorectomy, biomarker, biopsy, brachytherapy, brivanib, carbohydrate antigen 19.9 , carbohydrate antigen 125 , calreticulin, calretinin, carboplatin, caspase-3, CCNE1, CD44, CEA, cediranib, cell-free DNA, cervical cytology, chemoradiotherapy, chemotherapy, chitinase-3-like 1 protein, cisplatin, clinical examination, clinical manifestation, clinical staging, complex atypical hyperplasia, complications, comprehensive surgical staging, comprehensive staging, computed tomography, conservative surgery, conservative treatment, CTNNB1, curettage, Cyclin-dependent kinase 4/6, cyclooxygenase-2, cytology, cytoreduction, cytoreductive surgery, dalantercept, diagnosis, diagnostic performance, differential diagnosis, diffusion-weighted imaging, diffusion-weighted magnetic resonance imaging, dilatation and curettage, DJ-1, DNA integrity index,dovitinib, doxorubicin, dual-specific phosphatase 6, e-cadherin, early disease, early stage, endometrial biopsy, endometrial cancer, endometrial carcinoma, endometrial intraepithelial neoplasia, endometrial hyperplasia, endometrial sampling, endometrioid endometrial cancer, epithelial cell adhesion molecule, estrogen receptor, external beam radiation therapy, extra-fascial hysterectomy, everolimus, fertility, fertility outcome, fertility preservation, fertility sparing, fertility sparing management, fertility sparing surgery, fertility-preserving treatment, fibroblast growth factor receptor 2, follow-up, follow-up protocols, forkhead box protein 01, frozen section analysis, frozen section, FXYD3, gedatolisib, gene mutation testing, glucose-regulated protein 78, gonadotropin-releasing hormone, Gonadotropin-releasing hormone agonist, gross examination, health-related quality of life, hematopoietic pre-B-cell leukemia transcription factor-interacting protein, hereditary nonpolyposis colorectal cancer, hereditary nonpolyposis colorectal cancer syndrome, high-dose rate brachytherapy, hormonal therapy, hormone therapy, human epidermal growth factor receptor 2, human epididymis protein 4 , hyperthermic intraperitoneal chemotherapy, hysterectomy, hysteroscopy, hysteroscopic biopsy, hysteroscopic resection, imaging, immunohistochemical diagnosis, immunohistochemistry, intensity-modulated radiation therapy, interstitial brachytherapy, intervall debulking surgery, intracavity brachytherapy, intraoperative frozen section, Ki67, L1 cell adhesion molecule, laparoendoscopic single-site approach, laparoscopic staging, laparoscopy, laparotomy, late recurrence, levonorgestrel intrauterine device, levonorgestrel intrauterine system, local control, low dose rate brachytherapy, locally advanced cancer, lymphadenectomy, lymph node, lymph node assessment, lymph node dissection, lymph node involvement, lymph node staging, Lynch syndrome, magnetic resonance imaging, management, marker, maximum standardized uptake value, medroxyprogesterone, medroxyprogesterone acetate, megestrol acetate, MEK-1/2 inhibitor, metalloproteinase matrix 11, metalloproteinase matrix 2, metastatic disease, microcystic elongated fragmented pattern, micro RNA, microsatellite instability, mini-laparoscopic approach, mini-laparoscopic surgery, mini-laparoscopy, minimally invasive approach, minimally invasive surgery, mismatch repair, molecular biology, molecular marker, monocarboxylate Transporter 1, mortality rate, mortality analysis, mTOR inhibitor, multivariate analysis, mutL homolog $1, \mathrm{~N}$-arachidonoylethanolamine, napsin $\mathrm{A}$, neoadjuvant chemotherapy, neopterin, nintedanib, nodal involvement, $\mathrm{N}$-palmitoylethanolamine, nuclear receptor co-repressor, nuclear ubiquitous casein and cyclin-dependent kinases substrate, omentectomy, oral progestin therapy, oral progestogens, ovarian preservation, p300/CREB-binding protein, p16, p21, p53, p62, paclitaxel, Parkinson's disease-associated protein 7, pathology, paired box gene 2, paired box gene 8 , para-aortic lymph node, para-aortic lymphadenectomy, pelvic exenteration, pelvic lymph node, pelvic lym phadenectomy, peptide inhibitor, percutaneous surgery, percutaneous surgical system, perifosine, perioperative care, peritoneal cytology, phosphatase and tensin homolog, phospho-mTOR, physical examination, PI3K inhibitor, pilaralisib, polymerase epsilon, positron emission tomography, positron emission tomography-computed tomography, postoperative care, postoperative complications, postoperative recurrence, preoperative care, preoperative staging, preoperative work-up, progesterone receptor, progestin, prognosis, prognostic factor, prognostic value, programmed cell death ligand-1, proliferative endometrium, prophylactic hysterectomy, prophylactic surgery, quality of health care, quality of life, radiation therapy, radical hysterectomy, radiotherapy, rapalogs, Ras association domain family 1 isoform A, recurrence, recurrent disease, relapse, reoperation, residual disease, residual tumour, restaging, ridaforolimus, risk factors, risk groups, robot-assisted surgery, robotic laparoendoscopic single-site approach, robotic approach, robotic surgery, salpingectomy, salvage chemotherapy, salvage intraperitoneal chemotherappy, salvage radiation therapy, salvage radiotherapy, sandwich adjuvant chemotherapy, sandwich chemo-radiotherapy, sandwich method, sandwich radiation, salvage surgery, salvage treatment, screening, second line chemotherapy, second line treatment, selumetinib, sensitivity, sentinel lymph node, sex-determining region Y-box 2, side effects, silencing mediator for retinoid and thyroid-hormone receptors, sentinel lymph node dissection, sentinel lymph node mapping, snail, specificity, staging, staging procedures, standardized uptake value, stathmin,steroid receptor coactivator-1, sunitinib, surgery, surgical management, surgical outcome, surgical outcome criteria, surgical procedures, surgical resection, surveillance, survival, survival rate, survival analysis, systematic lymphadenectomy, tamoxifen, targeted therapy, taxane, temsirolimus, thyroid transcription factor-1, toxicity, transglutaminase 2, transvaginal ultrasound, treatment outcome, trebananib, tyrosinekinase inhibitor, ubiquitin-specific protease 14, ultra minimally invasive approach, ultra minimally invasive surgery, ultrasonography, unilateral salpingo-oophorectomy, vaginal brachytherapy, vascular endothelial growth factor, vascular endothelial growth factor antibody, vimentin, weight loss, weight loss interventions, weight reduction, Wilms tumour 1, workup, YKL-40.

Language English

Study design Priority was given to high-quality systematic reviews, meta-analyses, and randomised controlled trials but lower levels of evidence were also evaluated. The search strategy excluded editorials, letters, case reports and in vitro studies. The reference list of each identified article was reviewed for other potentially relevant papers. 


\section{APPENDIX 2. LIST OF THE 191 EXTERNAL REVIEWERS}

Kasimu Adoke, pathologist (Nigeria); Kamal Akbarov, radiation oncologist (Azerbaijan); Cherif Akladios, gynecologic oncologist (France); Moiad Alazzam, gynecologic oncologist (United Kingdom); Anastazija Aleksandrova Stanojevic, radiation oncologist (Croatia); Giovanni Aletti, gynecologic oncologist (Italy); Roberto Altamirano, gynecologic oncologist (Chile); Igor Aluloski, gynecologic oncologist (Macedonia); Frederic Amant, gynecologic oncologist (The Netherlands); Evsei Anca, pathologist (Romania); Maarit Anita Anttila, gynecologic oncologist (Finland); David Atallah, gynecologic oncologist (Lebanon); Beyhan Ataseven, gynecologic oncologist (Germany); Annika Auranen, gynecologic oncologist (Finland); Manel Barahona Orpinell, gynecologic oncologist (Spain); Maria-Pilar Barretina-Ginesta, medical oncologist (Spain); Marco Johannes Battista, gynecologic oncologist (Germany); Margarida Bernardino, gynecologic oncologist (Portugal); Rasiah Bharathan, gynecologic oncologist (United Kingdom); Mariusz Bidzinski, gynecologic oncologist (Poland); Claire Bonneau, gynecologic oncologist (France); Jacky Botterman, clinical oncologist (Belgium); Elena Ioana Braicu, gynecologist (Germany); Kjersti Bruheim, radiation oncologist (Norway); Alessandro Buda, obstetrician gynecologist (Italy); Katharina Buser, medical oncologist (Switzerland); Donato CallegaroFilho, medical oncologist (Brazil); Alessia Cimadamore, pathologist (Italy); Rachel Cooper, radiation oncologist (United Kingdom); Ovidiu Florin Coza, radiation oncologist (Romania); Melissa Christiaens, radiation oncologist (Belgium); Alessandro D'Amuri, pathologist (Italy); Caetano Da Silva Cardinal, gynecologic oncologist (Brazil); Christian Dannecker, obstetrician gynecologist (Germany); Nagindra Das, gynecologic oncologist (United Kingdom); Shatavisha Dasgupta, pathologist (India); Ben Davidson, pathologist (Norway); Diederick De Jong, gynecologic oncologist (United Kingdom); Cor De Kroon, gynecologic oncologist (The Netherlands); Hannelore Denys, medical oncologist (Belgium); Berta DiazFeijoo, gynecologic oncologist (Spain); Johannes Dimopoulos, radiation oncologist (Greece); Santiago Domingo, gynecologic oncologist (Spain); Catriona Doyle, patient (Ireland); Catherine Durdux, radiation oncologist (France); Sheila Elmes, patient (Ireland); Gemma Eminowicz, clinical oncologist (United Kingdom); Ane Gerda Zeriksson, gynecologic oncologist (Norway); Serkan Erkanli, gynecologic oncologist (Turkey); Henrik Falconer, gynecologic oncologist (Sweden); Daniela Fanni, pathologist (Italy); Annamaria Ferrero, gynecologic oncologist (Italy); Daniela Fischerova, gynecologic oncologist (Czech Republic); Anne Floquet, medical oncologist (France); Cristina Frutuoso, gynecologic oncologist (Portugal); Antonia Furtado, pathologist (Portugal); Luca Fuso, obstetrician gynecologist (Italy); Ketankumar Gajjar, gynecologic oncologist (United Kingdom); Isabella Maria Giovanna Garassino, medical oncologist (Italy); Christine Gennigens, medical oncologist (Belgium); Prafull Ghatage, gynecologic oncologist (Canada); Elpida-Linda Giannikaki, pathologist (Greece); Antonio Gil-Moreno, gynecologic oncologist (Spain); Laurence Gladieff, medical oncologist (France); Mikel Gorostidi, gynecologic oncologist (Spain); Perry Grigsby, radiation oncologist (United States of America); Christoph Grimm, gynecologic oncologist (Austria); Karin Grisan, clinical oncologist (Estonia); Esther Guerra Fernandez, pathologist (Spain); Kristensen Gunnar, gynecologic oncologist (Norway); Christine HaieMeder, radiation oncologist (France); Herman Haller, obstetrician gynecologist (Croatia); David Hardisson, pathologist (Spain); Annette Hasenburg, gynecologic oncologist (Germany); Gines Hernandez Cortes, obstetrician gynecologist (Spain); Fernanda Herrera, radiation oncologist (Switzerland); Cathrine Holland, gynecologic oncologist (United Kingdom); Peter Hoskin, clinical oncologist (United Kingdom); Arunachalam Ilancheran, gynecologic oncologist (Singapore); LeteInaki, gynecologic oncologist (Spain); Ibon Jaunarena, gynecologic oncologist (Spain); Kirsten Marie Jochumsen, gynecologist (Denmark); Florence Joly, medical oncologist (France); Ina JurgenliemkSchul, radiation oncologist (The Netherlands); Ioannis Kalogiannidis, gynecologic oncologist (Greece); Deni Karelovic, gynecologic oncologist (Croatia); Vesna Kesić, gynecologic oncologist (Serbia); Pearly Khaw, radiation oncologist (Australia); Gurkan Kiran, gynecologic oncologist (Turkey); AlexandraTimea Kirsch-Mangu, radiation oncologist (Romania); Jaroslav Klat, gynecologic oncologist (Czech Republic); Heinz Kölbl, gynecologic oncologist (Austria); Zoard Tibor Krasznai, obstetrician gynecologist (Hungary); Antonio Lagoa, gynecologist (Portugal); Joel Laufer, gynecologist (Uruguay); 
Naomi Lavan, radiation oncologist (Ireland); Kimseng Law, gynecologic oncologist (Taiwan); Jacob Christian Lindegaard, clinical oncologist (Denmark); Chien-Ting Liu, medical oncologist (Taiwan); Mathieu Luyckx, gynecologic oncologist (Belgium); Jose Claudio Maanon, obstetrician gynecologist (Spain); Sven Mahner, gynecologic oncologist (Germany); Suzana Manxhuaka-Kerliu, pathologist (Kosovo); Jose Maria Mariconde, gynecologic oncologist (Argentina); Claudia Mateoiu, pathologist (Sweden); Visnja Matkovic, gynecologic oncologist (Croatia); Mary McCormack, clinical oncologist (United Kingdom); Juan Manuel Medina-Castro, gynecologic oncologist (Mexico); Santosh Menon, gynecologic oncologist (India); Sebastjan Merlo, gynecologic oncologist (Slovenia); Nadav Michaan, gynecologic oncologist (Israel); Swarupa Mitra, radiation oncologist (India); Milos Mlyncek, gynecologic oncologist (Slovakia); Ole Mogensen, gynecologic oncologist (Denmark); Sabina Murshudova, gynecologic oncologist (Azerbaijan); Alexander Mustea, obstetrician gynecologist (Germany); Eva Myriokefalitaki, gynecologic oncologist (United Kingdom); Henrique Nabais, gynecologic oncologist (Portugal); Esten Nakken, radiation oncologist (Sweden); Gregg Nelson, gynecologic oncologist (Canada); Eva-Maria Niine-Roolaht, gynecologic oncologist (Estonia); Natalia Niziaeva, pathologist (Russia); Ines Nobre-Gois, radiation oncologist (Portugal); Felipe Ojeda, obstetrician gynecologist (Spain); Maja Pakiz, gynecologic oncologist (Slovenia); Patricia Pautier, medical oncologist (France); Fedro Alessandro Peccatori, obstetrician gynecologist (Italy); Anna Myriam Perrone, gynecologist (Italy); Anna Pesci, pathologist (Italy); Suzana Pessini, gynecologic oncologist (Brazil); Johanna Pijnenborg, gynecologic oncologist (The Netherlands); Kazimierz Pitynski, gynecologic oncologist (Poland); Stephan Polterauer, gynecologic oncologist (Austria); Jordi Ponce, gynecologist (Spain); Olga Ponomarova, medical oncologist (Ukraine); Melanie Powell, clinical oncologist (United Kingdom); Jiri Presl, gynecologic oncologist (Czech Republic); Mario Preti, gynecologist (Italy); Khalil Razvi, gynecologic oncologist (United Kingdom); Mikulas Redecha, gynecologic oncologist (Slovakia); Alexander Reinthaller, gynecologic oncologist (Austria); Vera Ribeiro, gynecologist (Portugal); Freydun Ronaghi, gynecologic oncologist (Austria); Ramon Rovira, gynecologic oncologist (Spain); Angeles Rovirosa, radiation oncologist (Spain); Vilius Rudaitis, gynecologist (Lithuania); Mameri Saadia Houria, pathologist (Algeria); Andres Sacristan, obstetrician gynecologist (Spain); Vanda Salutari, gynecologic oncologist (Italy); Marco Sanchez, gynecologic oncologist (Peru); Apostolos Sarivalasis, medical oncologist (Switzerland); Christian Schauer, gynecologic oncologist (Austria); Maximilian Schmid, radiation oncologist (Austria); Dietmar Schmidt, pathologist (Germany); Susy Marie Elisabeth Scholl, clinical oncologist (France); Yakir Segev, gynecologic oncologist (Israel); Paul Sevelda, gynecologic oncologist (Austria); Aliyev Shamistan, gynecologic oncologist (Azerbaijan); Tayup Simsek, gynecologic oncologist (Turkey); Shalini Singh, radiation oncologist (India); Vasileios Sioulas, gynecologic oncologist (Greece); Dounia Skalli Chrisostome, gynecologist (France); Erik SoegaardAndersen, gynecologic oncologist (Denmark); Synnöve Staff, gynecologic oncologist (Finland); Simona Stolnicu, pathologist (Romania); Gavin Charles Edward Stuart, gynecologic oncologist (Canada); Maciej Stukan, gynecologic oncologist (Poland); Li Tee Tan, clinical oncologist (United Kingdom); Rafal Tarkowski, gynecologic oncologist (Poland); Cagatay Taskiran, gynecologic oncologist (Turkey); Maria Topalidou, radiation oncologist (Greece); Helen Trihia, pathologist (Greece); Philippe Tummers, gynecologic oncologist (Belgium); Katrien Vandecasteele, radiation oncologist (Belgium); Jacobusvan der Velden, gynecologic oncologist (The Netherlands); Koen van de Vijver, pathologist (Belgium); Toon van Gorp, gynecologic oncologist (Belgium); Rasa Vanseviciute Petkeviciene, gynecologic oncologist (Lithuania); Ignacio Vazquez, medical oncologist (United Kingdom); August Vidal, pathologist (Spain); Nadia Villena Salinas, pathologist (Denmark); David Wachter, pathologist (Germany); Nicola Weidner, radiation oncologist (Germany); Eva Weis, radiation oncologist (Austria); Henrica MJ Werner, gynecologic oncologist (The Netherlands); Henrike Westerveld, radiation oncologist (The Netherlands); Jacek Wilczynski, gynecologic oncologist (Poland); Oda Petronela Witteveen, medical oncologist (The Netherlands); Paulo Zanvettor, gynecologic oncologist (Brazil); Alain Zeimet, gynecologic oncologist (Austria); Paolo Zola, gynecologic oncologist (Italy). 